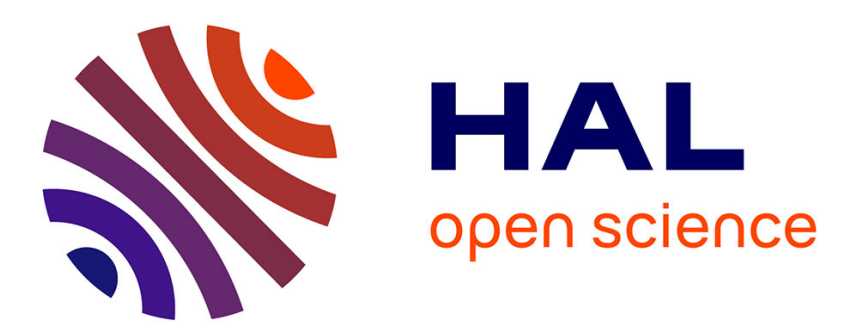

\title{
Managing Inequality over Business Cycles: Optimal Policies with Heterogeneous Agents and Aggregate Shocks
}

François Le Grand, Xavier Ragot

\section{To cite this version:}

François Le Grand, Xavier Ragot. Managing Inequality over Business Cycles: Optimal Policies with Heterogeneous Agents and Aggregate Shocks. International Economic Review, In press, 63 (1), pp.511540. 10.1111/iere.12537 . hal-03501381

\section{HAL Id: hal-03501381 \\ https://hal-sciencespo.archives-ouvertes.fr/hal-03501381}

Submitted on 30 Dec 2021

HAL is a multi-disciplinary open access archive for the deposit and dissemination of scientific research documents, whether they are published or not. The documents may come from teaching and research institutions in France or abroad, or from public or private research centers.
L'archive ouverte pluridisciplinaire HAL, est destinée au dépôt et à la diffusion de documents scientifiques de niveau recherche, publiés ou non, émanant des établissements d'enseignement et de recherche français ou étrangers, des laboratoires publics ou privés.

\section{(ㅇ)(1) $\$$}

Distributed under a Creative Commons Attribution - NonCommercial - NoDerivatives| 4.0 


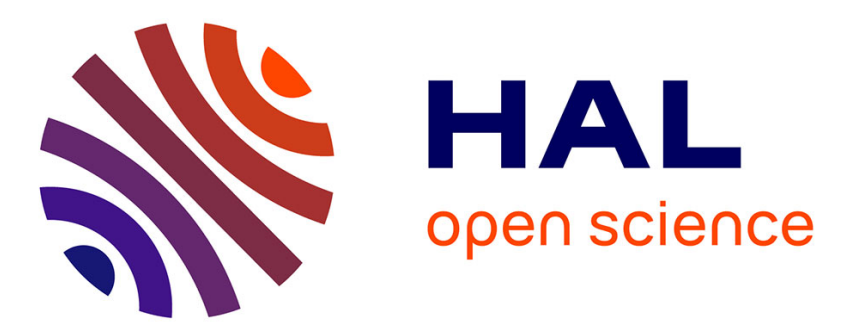

\section{Managing Inequality over Business Cycles: Optimal Policies with Heterogeneous Agents and Aggregate Shocks}

François Le Grand, Xavier Ragot

\section{- To cite this version:}

François Le Grand, Xavier Ragot. Managing Inequality over Business Cycles: Optimal Policies with Heterogeneous Agents and Aggregate Shocks. International Economic Review, Wiley, In press, 10.1111/iere.12537 . hal-03501381

HAL Id: hal-03501381

https://hal-sciencespo.archives-ouvertes.fr/hal-03501381

Submitted on 30 Dec 2021

HAL is a multi-disciplinary open access archive for the deposit and dissemination of scientific research documents, whether they are published or not. The documents may come from teaching and research institutions in France or abroad, or from public or private research centers.
L'archive ouverte pluridisciplinaire HAL, est destinée au dépôt et à la diffusion de documents scientifiques de niveau recherche, publiés ou non, émanant des établissements d'enseignement et de recherche français ou étrangers, des laboratoires publics ou privés. 


\title{
Managing Inequality over Business Cycles: Optimal Policies
}

\author{
with Heterogeneous Agents and Aggregate Shocks ${ }^{1}$
}

\author{
François Le Grand Xavier Ragot ${ }^{2}$
}

July 8, 2021

\begin{abstract}
We present a truncation theory of idiosyncratic histories for heterogeneous-agent models. This method allows us to solve for optimal Ramsey policies in such models with aggregate shocks. The method can be applied to a large variety of settings, with occasionally binding credit constraints. We use this theory to characterize the optimal level of unemployment insurance over the business cycle in a production economy. We find that the optimal policy is countercyclical.
\end{abstract}

Keywords: Incomplete markets, optimal policies, heterogeneous-agent models, Ramsey program.

JEL codes: E21, E44, D91, D31.

\footnotetext{
${ }^{1} \mathrm{~A}$ previous version of the paper was circulated as "Optimal fiscal policy with heterogeneous agents and aggregate shocks". We thank Dirk Krueger for excellent suggestions on the previous versions of the paper. We are also grateful to Kurt Mitman for having shared his code with us and to Wouter Den Haan and Albert Marcet for their useful suggestions. We also thank Édouard Challe, Varadarajan Chari, Jonathan Heathcote, Christos Koulovatianos, Felix Kubler, Étienne Lehmann, Alaïs Martin-Baillon, Ben Moll, Fabrizio Perri, Elisabeth Pröhl, Morten Ravn, Michael Reiter, Diego Rodrigues, Stephanie Schmitt-Grohe, Vincent Sterck, Martin Uribe, Gianluca Violante, Pierre-Olivier Weill, Sevin Yeltekin, and the seminar participants at the Federal Reserve Bank of Minneapolis, University of Zurich, University of Luxembourg, University of Cambridge, New York University, National Bank of Belgium, and European University Institute, at the "Macroeconomics of aggregate fluctuations and stabilization policy" conference, the T2M 2019, EEA 2018, SED 2019, and NBER SI 2017 MPF conference at the RED Conference in Barcelona. We thank Sumudu Kankamange and Thomas Weitzenblum for their excellent research assistance. The research leading to these results received funding from the European Community's Seventh Framework Programme (FP7/2007-2013) under grant agreement Integrated Macro-Financial Modeling for Robust Policy Design (MACFINROBODS, grant no. 612796).

${ }^{2}$ Le Grand: emlyon business school and ETH Zurich; legrand@em-lyon.com. Ragot: Sciences Po, OFCE and CNRS; xavier.ragot@gmail.com.
} 


\section{Introduction}

Incomplete insurance market economies provide a useful framework for examining many relevant aspects of inequalities and individual risks. In these models, infinitely-lived agents face incomplete insurance markets and borrowing limits that prevent them from perfectly hedging their idiosyncratic risk, in line with the Bewley-Huggett-Aiyagari literature (Bewley, 1983; Imrohoroğlu, 1989; Huggett, 1993; Aiyagari, 1994; Krusell and Smith, 1998). These frameworks are now widely used, since they fill a gap between micro- and macroeconomics, and enable the inclusion of aggregate shocks and a number of additional frictions on both the goods and labor markets. However, little is known about optimal policies in these environments due to the difficulties generated by the large and timevarying heterogeneity across agents. This is unfortunate, since a vast literature suggests that the interaction between wealth heterogeneity and capital accumulation has first-order implications for the design of optimal policies. An important example is the optimal design of time-varying unemployment benefits in an economy with fluctuating unemployment risk, which has not yet been studied in the general case due to the difficulties generated by the variations in precautionary savings over the business cycle.

We present a general method that allows one to characterize optimal policy in models with household heterogeneity and aggregate shocks. In standard incomplete insurance market economies, agents differ according to the full history of their idiosyncratic risk realizations. Huggett (1993) and Aiyagari (1994), using the results of Hopenhayn and Prescott (1992), have shown that economies without aggregate risk have a recursive structure when the distribution of wealth is introduced as a state variable. Unfortunately, the distribution of wealth has an infinite number of possible values, which hinders the analysis of optimal policies in these types of environment.

The main idea of our computational method is to go back to the sequential formulation of incomplete-market models to construct a consistent finite state-space representation. We proceed in three steps. First, we construct a partition in the space of idiosyncratic histories, using a truncation procedure. For a given truncation length $N$, all agents with the same idiosyncratic history over the last $N$ periods are grouped together. Second, along the truncated histories, the allocations of the original model are expressed in terms of 
aggregate variables (i.e., groups of agents sharing the same N-period history), rather than individual ones. ${ }^{3}$ This allows us to provide an exact aggregation theory of the model with aggregate shocks. ${ }^{4}$ This results in a so-called exact aggregated model, which is an equivalent representation of the original model and does not involve any simplifying assumptions. Finally, from this aggregated model, we construct a truncated model in the presence of aggregate shocks, based on one additional simplifying assumption: the within-history heterogeneity is assumed to remain constant in the dynamics. This assumption preserves the within-history steady-state heterogeneity, but it do not let it vary with aggregate shocks. This within-history heterogeneity is shown to vanish when the truncation length becomes infinitely long and turns out to have little quantitative impact. The truncated model thus focuses on the dynamics of heterogeneity across truncated histories.

The interest of this construction is twofold. First, we can prove that the Ramsey allocation of the truncated model converges to the allocation of the original incompletemarket model when the truncation length becomes infinitely long. ${ }^{5}$ To do so, we use the tools developed in dynamic contracts, sometimes referred to as the Lagrangian approach and developed by Marcet and Marimon (2019) among others. ${ }^{6}$ We show how the Lagrangian approach must be adapted to deal with our model, which features occasionally-binding credit constraints. This convergence result proves that the truncation methodology provides a consistent representation. Second, our methodology provides a simple numerical procedure to solve for Ramsey allocations both at the steady state and with aggregate shocks. In particular, the finite state-space structure allows us to use linear algebra to compute steady-state values of Lagrange multipliers in closed-form. The dynamics can thus be simulated with perturbation methods and rely on standard software, such as Dynare (Adjemian et al., 2011). We quantitatively find that a short truncation length yields an accurate solution.

\footnotetext{
${ }^{3}$ Original model refers to the original baseline heterogeneous-agent model, expressed in terms of individual agents.

${ }^{4}$ More general partitions - based on other criteria than the truncation - could be considered in a very similar way. This could rely on any criterion guaranteeing a partition of the space of idiosyncratic histories.

${ }^{5} \mathrm{We}$ also check that, in terms of numerical performance, the truncation method delivers dynamics similar to those implied by other numerical techniques, in particular those of Reiter (2009) and Boppart et al. (2018).

${ }^{6}$ Our methodology relies only on some elements of the Lagrangian approach. For instance, it does not use the recursive formulation, which avoids further complexity (see Cole and Kubler, 2012, and Pavoni et al., 2018 for a discussion).
} 
We use our truncation theory to characterize optimal unemployment benefits over the business cycle in an economy where agents face both productivity risk and time-varying employment risk, as in Krueger et al. (2018). The economy is hit by aggregate shocks that affect technology and labor market transitions. Agents choose their labor supply when working, consume, save, and face incomplete markets for idiosyncratic risk with credit constraints. In this economy, a planner chooses the level of unemployment benefits in each period, which must be fully financed by a distorting labor tax. Although the economic trade-off is the standard trade-off between insurance and efficiency, this problem is very hard to solve in a general equilibrium setting. The level of unemployment benefits directly affects agents' welfare, their saving decisions, as well as the dynamics of interest rates and wages. We find that the replacement rate is countercyclical, increasing the transfer to unemployed agents in recessions. This policy reduces the volatility of the total income of unemployed agents, which is welfare improving.

Literature review. Our paper contributes to the recent literature on optimal policies in heterogeneous-agent models, which can be split into three groups. First, some papers, such as Aiyagari and McGrattan (1998), focus on the maximization of the steady-state welfare. Second, other papers look for optimal policies while accounting for the cost of transitions (see for instance Krueger and Ludwig, 2016, Dyrda and Pedroni, 2018, or Chang et al., 2018). These papers directly maximize the aggregate intertemporal welfare over all possible paths of the planner's instruments. This method is computationally very intensive, which may limit the number of instruments used by the planner. Third, a last group of papers solves for the first-order conditions of the planner's program. Aiyagari (1995) pioneers the study of the Ramsey allocation in a general setup and characterizes the optimal capital tax. The use of Lagrange multipliers is further developed in Açikgöz et al. (2018). Nuño and Moll (2018) consider a continuous-time framework in which they use the techniques of Ahn et al. (2017) based on calculus of variation to characterize optimal monetary policy. All these papers solve for optimal policy without aggregate shocks. Our truncation method allows us to solve for optimal policies with no restrictions, both at the steady state and with aggregate shocks. To the best of our knowledge, the only paper deriving optimal Ramsey policy in a similarly general environment is Bhandari et al. (2020). 
Their method can account for large aggregate shocks but relies on a "primal approach" in which credit constraints can be always binding or never binding, but not occasionally binding. Compared to their model, our solution strategy works well with occasionally binding credit constraints, which may be the relevant case in some environments. ${ }^{7}$

Our paper also contributes to the literature on solution methods for incomplete insurance market economies with aggregate shocks. Our truncation method is related to other projection and perturbation methods (Rios-Rull, 1999, Reiter, 2009, and Young, 2010), which have been shown to be accurate approximations compared to global solution techniques (see Boppart et al., 2018 or Auclert et al., 2019). The main difference is that our solution is based on idiosyncratic histories and not on the space of wealth, which turns out to be helpful for solving Ramsey programs. In particular, our method keeps track of the relevant distribution, which is necessary for deriving optimal policies, because they depend on the distributive effects of the Ramsey instruments. Finally, our truncation of idiosyncratic histories is related to, but different from, the truncation of aggregate histories (see for instance Chien et al., 2011, 2012). Our truncation is used to derive a limited-heterogeneity representation of the original model, which we simulate using perturbation methods. To the best of our knowledge, this paper is the first to develop a truncation in the space of idiosyncratic histories.

Finally, regarding the application, our paper contributes to the literature on optimal unemployment benefits. This literature is huge, and a large part of it employs the sufficientstatistics approach (see the surveys of Chetty, 2009, Chetty and Finkelstein, 2013, and Kolsrud et al., 2018, for recent developments), based on partial equilibrium analysis. A handful of papers introduce general equilibrium effects, such as Mitman and Rabinovich (2015), Landais et al. (2018a,b), or Ábrahám et al. (2019), but they focus on labor market externalities and not on saving distortions. To the best of our knowledge, the only paper analyzing optimal unemployment insurance in general equilibrium with saving choices is

\footnotetext{
${ }^{7}$ Another strategy used in the literature is to focus on a simplified economy, in which the wealth distribution has a tractable structure. Some papers, such as Ravn and Sterk (2017), Challe (2020), and McKay and Reis (2020) follow Constantinides and Duffie (1996) and Krusell et al. (2011). They assume a zero-net supply for assets, which yields a no-trade equilibrium. Other papers, such as Bilbiie (2008), or Bilbiie and Ragot (2020), consider two-agent economies in which a second-order approximation of the welfare function can be derived. Finally, a last group of papers, such as Acharya et al. (2020), consider a CARA-normal economy without binding credit constraint, which enables to derive aggregate dynamics. All these models provide important economic insights but they rely on special cases. Their quantitative relevance is thus hard to assess.
} 
Krusell et al. (2010). To simplify the quantitative exercise, the authors perform a welfare analysis by comparing steady states with different levels of unemployment benefits. We adopt a different approach, deriving the time-varying solution of a general Ramsey problem in an economy with aggregate shocks.

The rest of the paper is organized as follows. In Section 2 we present the environment. In Section 3 we derive optimal Ramsey policies and discuss the economic trade-off for optimal unemployment benefits over the business cycle. In Section 4 we construct the truncated model and provide convergence properties. Section 5 sets out our quantitative analysis. Finally, Section 6 compares our method in terms of accuracy to those of Reiter (2009) and Boppart et al. (2018).

\section{The economy}

Time is discrete and indexed by $t=0,1,2, \ldots$ The economy is populated by a continuum of agents of measure 1 , distributed on an interval $\mathcal{I}$ according to a measure $\ell(\cdot)$. We follow Green (1994) and assume that the law of large numbers holds.

\subsection{Preferences}

In each period, agents derive utility from private consumption $c$ and disutility from labor

$l$. The period utility function, denoted by $U(c, l)$, is assumed to be of the GreenwoodHercowitz-Huffman (GHH) type, exhibiting no wealth effect for the labor supply, as in Heathcote (2005), for instance:

$$
U(c, l)=u\left(c-\chi^{-1} \frac{l^{1+1 / \varphi}}{1+1 / \varphi}\right),
$$

where $\varphi>0$ is the Frisch elasticity of labor supply, $\chi>0$ scales labor disutility, and $u: \mathbb{R}_{+} \rightarrow \mathbb{R}$ is twice continuously derivable, increasing, and concave, with $u^{\prime}(0)=\infty$ and $u^{\prime}(\infty)=0$. Our results do not rely on the GHH functional form, and we could consider a more general utility function $U$. The algebra is simplified, however, especially in the Ramsey program, because of the absence of a wealth effect for the labor supply. In the online appendix (Section E.3), we show how to use our truncation method with a more general utility function. 
Agents have standard additive intertemporal preferences, with a constant discount factor $0<\beta<1$. They therefore rank consumption and labor streams, denoted respectively by $\left(c_{t}\right)_{t \geq 0}$ and $\left(l_{t}\right)_{t \geq 0}$, using the intertemporal utility criterion $\sum_{t=0}^{\infty} \beta^{t} U\left(c_{t}, l_{t}\right)$.

\subsection{Risks}

We consider a general setup where agents face an aggregate risk, a time-varying unemployment risk, and a productivity risk, as modeled by Krueger et al. (2018). As will be clear in the quantitative analysis below, this general setup allows us to match realistic labor market wealth distribution and dynamics. ${ }^{8}$

Aggregate risk. The aggregate risk affects both aggregate productivity and unemployment risk. At a given date $t$, the aggregate state is denoted by $z_{t}$ and takes values in the (possibly continuous) state space $\mathcal{Z} \subset \mathbb{R}^{+}$. We assume that the aggregate risk is a Markov process. The history of aggregate shocks up to time $t$ is denoted by $z^{t}=\left\{z_{0}, \ldots, z_{t}\right\} \in \mathcal{Z}^{t+1}$. For the sake of clarity, we will denote the realization of any random variable $X_{t}: \mathcal{Z}^{t+1} \rightarrow \mathbb{R}$ in state $z^{t}$ by $X_{t}$, instead of $X_{t}\left(z^{t}\right)$, when there is no ambiguity.

Employment risk. At the beginning of each period, each agent $i \in \mathcal{I}$ faces an uninsurable idiosyncratic employment risk, denoted by $e_{t}^{i}$ at date $t$. The employment status $e_{t}^{i}$ can take two values, $e$ and $u$, corresponding to employment and unemployment, respectively. We denote the set of possible employment statuses by $\mathcal{E}=\{e, u\}$. An employed agent with $e_{t}^{i}=e$ can freely choose her labor supply $l_{t}^{i}$. An unemployed agent with $e_{t}^{i}=u$ cannot work and will receive an unemployment benefit financed by a distorting tax on labor and will suffer from a fixed disutility reflecting a domestic effort. These aspects are further described below.

The employment status $\left(e_{t}^{i}\right)_{t \geq 0}$ follows a discrete Markov process with transition matrix $\Pi\left(z^{t}\right) \in[0,1]^{2 \times 2}$ - that will simply denoted as $\Pi_{t}-$, which is assumed to depend on the history of aggregate shocks up to date $t$. The job-separation rate between periods $t-1$ and $t$ is denoted by $\Pi_{t, e u}=1-\Pi_{t, e e}$, while $\Pi_{t, u e}=1-\Pi_{t, u u}$ is the job-finding rate between

\footnotetext{
${ }^{8}$ Compared to Krueger et al. (2018), we introduce endogenous labor supply, such that labor taxes are distorting. In addition, we simplify the economy and remove the age dimension. We follow these authors and denote all transition probabilities by $\Pi$ - which will be distinguished by their subscripts.
} 
$t-1$ and $t$. We denote the implied population shares of unemployed and employed agents by $S_{t, u}$ and $S_{t, e}$, respectively, with $S_{t, u}+S_{t, e}=1$.

Productivity risk. Agents' individual productivity, denoted by $y_{t}^{i}$, is stochastic and takes values in a finite set $\mathcal{Y} \subset \mathbb{R}_{+}$. Large values in $\mathcal{Y}$ correspond to high productivities. The before-tax wage earned by an employed agent $i$ is the product of the aggregate wage $w_{t}$ (dependent on aggregate shock), the labor effort $l_{t}^{i}$, and individual productivity $y_{t}^{i}$. The total before-tax wage is therefore $y_{t}^{i} w_{t} l_{t}^{i}$. An unemployed agent will also carry an idiosyncratic productivity level that will affect her unemployment benefits and her disutility level, denoted by $\zeta_{y}$ (for productivity $y \in \mathcal{Y}$ ), associated with domestic production.

The productivity status follows a first-order Markov process where the transition probability from state $y_{t-1}^{i}=y$ to $y_{t}^{i}=y^{\prime}$ is constant and denoted by $\Pi_{y y^{\prime}}$. In particular, it is independent of the agent's employment status. We denote by $S_{y}$ the share of agents endowed with individual productivity level $y$. This share is constant over time because of the assumptions regarding transition probabilities $\left(\Pi_{y y^{\prime}}\right)$.

The individual state of any agent $i$ is characterized by her employment status and her productivity level. We denote by $s_{t}^{i}=\left(e_{t}^{i}, y_{t}^{i}\right)$ the date- $t$ individual status of any agent, whose possible values lie in the set $\mathcal{S}=\mathcal{E} \times \mathcal{Y}$. Finally, we denote by $s^{i, t}=\left\{s_{0}^{i}, \ldots, s_{t}^{i}\right\}$ a history until period $t$. We can then use the transition probabilities for employment and productivity to derive the measure $\mu_{t}: \mathcal{S}^{t+1} \rightarrow[0,1]-\mu_{t}\left(s^{t}\right)$ being the measure of agents with history $s^{t}$ in period $t$.

\subsection{Production}

The good is produced by one profit-maximizing representative firm. This firm is endowed with production technology that transforms, at date $t$, labor $L_{t}$ (in efficient units) and capital $K_{t-1}$ into $Y_{t}$ output units of the single good. The production function $F$ is a Cobb-Douglas function with parameter $\alpha \in(0,1)$ featuring constant returns-to-scale. Capital must be installed one period before production and the total productivity factor $Z_{t}$ is stochastic. Constant capital depreciation is denoted by $\delta>0$, and net output $Y_{t}$ is 
formally defined as follows:

$$
Y_{t}=F\left(Z_{t}, K_{t-1}, L_{t}\right)=Z_{t} K_{t-1}^{\alpha} L_{t}^{1-\alpha}-\delta K_{t-1}
$$

where the total productivity factor is the exponential of the aggregate shock $z_{t}: Z_{t}=$ $\exp \left(z_{t}\right)$.

The two factor prices at date $t$ are the aggregate before-tax wage rate $w_{t}$ and the capital return $r_{t}$. The profit maximization of the producing firm implies the following factor prices:

$$
w_{t}=F_{L}\left(Z_{t}, K_{t-1}, L_{t}\right) \text { and } r_{t}=F_{K}\left(Z_{t}, K_{t-1}, L_{t}\right) .
$$

\subsection{Unemployment insurance}

A benevolent government manages an unemployment insurance (UI) scheme, in which labor taxes are raised to finance unemployment benefits. As labor supply is endogenous, labor tax is distorting. The government thus faces the standard trade-off between efficiency and insurance.

At any date $t$, unemployed agents receive an unemployment benefit that is equal to a constant fraction of the wage the agent would earn if she were employed (with the same productivity level). The replacement rate is denoted by $\phi_{t}$ and the unemployment benefit of an agent endowed with productivity $y_{t}$ equals $\phi_{t} w_{t} y_{t} \bar{l}_{t}\left(y_{t}\right)$, where $\bar{l}_{t}\left(y_{t}\right)$ is the average labor supply of employed agents with productivity $y_{t}$. We follow Krueger et al. (2018) for this specification, which usefully reduces the state space. From the agents' perspective, the replacement rate is an exogenous process that depends on the aggregate state $\phi_{t}=\phi_{t}\left(z^{t}\right)$.

Unemployment benefits are financed solely by the labor tax, which is only paid by employed agents. Taxes amount to a constant share $\tau_{t}$ of employed agents' wages with this proportion being identical for all employed agents. The contribution $\tau_{t}$ is set such that the UI scheme budget is balanced at any date $t$, no social debt being allowed:

$$
\phi_{t} w_{t} \int_{i \in \mathcal{U}_{t}} y_{t}^{i} l_{t}^{i}\left(y_{t}^{i}\right) \ell(d i)=\tau_{t} w_{t} \int_{i \in \mathcal{I} \backslash \mathcal{U}_{t}} y_{t}^{i} l_{t}^{i} \ell(d i)
$$

where $\mathcal{U}_{t} \subset \mathcal{I}$ is the set of unemployed agents at $t$ and $\mathcal{I} \backslash \mathcal{U}_{t}$ is the set of employed agents. 


\subsection{Agents' program and resource constraints}

We consider an agent $i \in \mathcal{I}$. She can save in an asset that pays the gross interest rate $1+r_{t}$. She is prevented from borrowing too much and her savings must remain above an exogenous threshold, $-\bar{a} \leq 0$. At date 0 , the agent chooses the consumption $\left(c_{t}^{i}\right)_{t \geq 0}$, labor supply $\left(l_{t}^{i}\right)_{t \geq 0}$, and saving plans $\left(a_{t}^{i}\right)_{t \geq 0}$ that maximize her intertemporal utility, subject to a budget constraint and the previous borrowing limit. Formally, for a given initial wealth $a_{-1}^{i}$, her program is: ${ }^{9}$

$$
\begin{aligned}
& \max _{\left\{c_{t}^{i}, l_{t}^{i}, a_{t}^{i}\right\}_{t=0}^{\infty}} \mathbb{E}_{0} \sum_{t=0}^{\infty} \beta^{t} u\left(c_{t}^{i}-\chi^{-1} \frac{l_{t}^{i, 1+1 / \varphi} 1_{e_{t}^{i}=e}+\zeta_{y_{t}^{i}}^{1+1 / \varphi} 1_{e_{t}^{i}=u}}{1+1 / \varphi}\right), \\
& c_{t}^{i}+a_{t}^{i}=\left(1+r_{t}\right) a_{t-1}^{i}+\left(\left(1-\tau_{t}\right) l_{t}^{i} 1_{e_{t}^{i}=e}+\phi_{t} \bar{l}_{t}\left(y_{t}^{i}\right) 1_{e_{t}^{i}=u}\right) y_{t}^{i} w_{t}, \\
& a_{t}^{i} \geq-\bar{a}, c_{t}^{i}>0, l_{t}^{i}>0 .
\end{aligned}
$$

Objective (5) accounts for the disutility of unemployed agents associated with domestic production. The budget constraint (6) is standard and the expression $\left(\left(1-\tau_{t}\right) l_{t}^{i} 1_{e_{t}^{i}=e}+\right.$ $\left.\phi_{t} \bar{l}_{t}\left(y_{t}^{i}\right) 1_{e_{t}^{i}=u}\right) y_{t}^{i} w_{t}$ is a compact formulation for the net wage (i.e., after taxes and unemployment benefits).

We denote by $\beta^{t} \nu_{t}^{i}$ the Lagrange multiplier on the credit constraint of agent $i$. The Lagrange multiplier is obviously null when the agent is not credit constrained. Taking advantage of the GHH utility function, the first-order conditions of an employed agent's program (5)-(7) are:

$$
\begin{aligned}
u^{\prime}\left(c_{t}^{i}-\chi^{-1} \frac{\hat{l}_{t}^{i, 1+1 / \varphi}}{1+1 / \varphi}\right) & =\beta \mathbb{E}_{t}\left[\left(1+r_{t+1}\right) u^{\prime}\left(c_{t+1}^{i}-\chi^{-1} \frac{\hat{l}_{t+1}^{i, 1+1 / \varphi}}{1+1 / \varphi}\right)\right]+\nu_{t}^{i}, \\
\left(l_{t}^{i}\right)^{1 / \varphi} & =\chi\left(1-\tau_{t}\right) w_{t} y_{t}^{i},
\end{aligned}
$$

where for all $t \geq 0$ and $i \in \mathcal{I}$, we introduce the notation $\hat{l}_{t}^{i}$ :

$$
\hat{l}_{t}^{i} \equiv l_{t}^{i} 1_{e_{t}^{i}=e}+\zeta_{y_{t}^{i}} 1_{e_{t}^{i}=u} .
$$

The GHH utility function implies that the labor supply in equation (9) only depends on current productivity and the after-tax real wage, which implies: $\bar{l}_{t}\left(y_{t}^{i}\right)=l_{t}^{i}$. Unem-

\footnotetext{
${ }^{9}$ In the remainder of the paper, $1_{A}$ will denote an indicator function equal to 1 if $A$ is true and 0 otherwise. For any $t \geq 0, \mathbb{E}_{t}$ will denote an expectation operator, conditional on the information available at date $t$.
} 
ployed agents have the same Euler equation (8). They supply no labor, but they earn unemployment benefits and suffer from disutility (terms in $\zeta_{y}$ ) related to home production.

We now turn to economy-wide constraints. Financial- and labor-market clearing implies the following relationships for the supply of capital $K_{t}$ and labor $L_{t}$ (in efficient units):

$$
\int_{i} a_{t}^{i} \ell(d i)=K_{t} \text { and } L_{t}=\int_{i \in \mathcal{I} \backslash \mathcal{U}_{t}} y_{t}^{i} l_{t}^{i} \ell(d i) \text {. }
$$

The clearing of the goods market implies that total consumption and the new capital stock equals total supply, itself the sum of output net of depreciation and past capital:

$$
\int_{i} c_{t}^{i} \ell(d i)+K_{t}=Y_{t}+K_{t-1}
$$

Using labor market transition probabilities, we deduce that the law of motion for the employed and unemployed agent populations, denoted respectively by $S_{t, e}$ and $S_{t, u}$, is:

$$
S_{t, u}=1-S_{t, e}=\Pi_{t, e u} S_{t-1, e}+\Pi_{t, u u} S_{t-1, u} .
$$

The constant share of agents $S_{y}$ with productivity $y$ verifies: $S_{y}=\sum_{y \in \mathcal{Y}} S_{y^{\prime}} \Pi_{y^{\prime} y}$.

Using individual labor Euler conditions (9), the UI budget constraint (4) can be written as: $\phi_{t} \int_{i \in \mathcal{U}_{t}}\left(y_{t}^{i}\right)^{1+\varphi} \ell(d i)=\tau_{t} \int_{i \in \mathcal{I} \backslash \mathcal{U}_{t}}\left(y_{t}^{i}\right)^{1+\varphi} \ell(d i)$. We observe that the budget balance only depends on the current idiosyncratic state. With equation (13), this can therefore be simplified into $\phi_{t} \sum_{y \in \mathcal{Y}} S_{t, u} S_{y} y^{1+\varphi}=\tau_{t} \sum_{y \in \mathcal{Y}} S_{t, e} S_{y} y^{1+\varphi}$, or:

$$
\phi_{t} S_{t, u}=\tau_{t} S_{t, e}
$$

We can now formulate our equilibrium definition.

Definition 1 (Sequential equilibrium) A competitive equilibrium is a collection of individual variables $\left(c_{t}^{i}, l_{t}^{i}, a_{t}^{i}, \nu_{t}^{i}\right)_{t \geq 0, i \in \mathcal{I}}$, of aggregate quantities $\left(K_{t}, L_{t}, Y_{t}\right)_{t \geq 0}$, of price processes $\left(w_{t}, r_{t}\right)_{t \geq 0}$, and of UI policy $\left(\tau_{t}, \phi_{t}\right)_{t \geq 0}$ such that, for an initial wealth distribution $\left(a_{-1}^{i}\right)_{i \in \mathcal{I}}$, and for initial values of capital stock $K_{-1}=\int_{i} a_{-1}^{i} \ell(d i)$, and of the aggregate shock $z_{-1}$, we have:

1. given prices, individual strategies $\left(c_{t}^{i}, l_{t}^{i}, a_{t}^{i}, \nu_{t}^{i}\right)_{t \geq 0, i \in \mathcal{I}}$ solve the agent's optimization program in equations (5)-(7);

2. financial, labor, and goods markets clear: for any $t \geq 0$, equations (11) and (12) 
hold;

3. the UI budget is balanced: equation (14) holds for all $t \geq 0$;

4. factor prices $\left(w_{t}, r_{t}\right)_{t \geq 0}$ are consistent with the firm's program (3).

\section{Ramsey program}

\subsection{Formulation of the Ramsey program}

The Ramsey problem involves determining the unemployment insurance policy (which consists here of the replacement rate $\phi_{t}$ and the labor tax rate $\tau_{t}$ ) that corresponds to the "best" competitive equilibrium, according to a utilitarian welfare criterion. Aggregate welfare is simply measured by the sum $\sum_{t=0}^{\infty} \beta^{t} \int_{i} U\left(c_{t}^{i}, l_{t}^{i}\right) \ell(d i)$. The Ramsey problem can thus be written as - with $\hat{l}_{t}^{i}$ defined in (10):

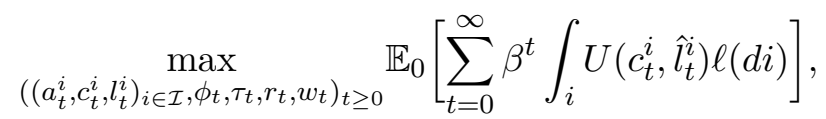

subject to: (i) individual budget constraints (6), (ii) the Euler equations (8) and (9) for consumption and labor, respectively, (iii) the UI scheme budget balance (14), (iv) the market clearing constraints (11), and finally (v) the factor prices definitions (3).

The Ramsey program of equation (15) features a concave objective but non-affine equality constraints (due to the presence of Euler equations). This implies that the first-order conditions (FOCs) of the Ramsey program may not be necessary nor sufficient to characterize the optimal policy. This limitation is shared with most of the literature on Ramsey programs, including papers featuring a representative-agent. Following the literature, we assume that the solution is interior and that the FOCs of the planner are necessary. Finally, note that the structure of our problem does not require to use a recursive formulation, which avoids further complexity (see Cole and Kubler, 2012 and Pavoni et al., 2018, among others).

A reformulation of the Ramsey problem. We simplify the formulation of the Ramsey problem exposed in equation (15), using the factorization of the Lagrangian employed by Marcet and Marimon (2019). However, two specific difficulties arise when 
applying this approach in our environment. The first difficulty is the consistency of the method with incomplete-market models. In fact, we show in Section B.1 of the online appendix that our setup adds no complexity to the formulation of the planner's objective. The second difficulty is the application of Marcet and Marimon (2019) to models with occasionally binding credit constraints. To show that the first-order conditions of the Lagrangian approach are valid, we derive an additional theoretical result in Proposition 6 of the online appendix (Section E.2), where we prove that the first-order conditions of our Ramsey problem can be understood as the limit of those of a Ramsey problem featuring penalty functions (whose concavity become infinitely high). Since penalty functions substitute for credit constraints, this result shows that Marcet and Marimon (2019)'s result still applies with occasionally binding credit constraints. ${ }^{10}$

We denote by $\beta^{t} \lambda_{t}^{i}$ the Lagrange multiplier of the consumption Euler equation (8) for agent $i$ at date $t$. Observing that the multiplier $\lambda_{t}^{i}$ is null when the credit constraint is binding for agent $i$. This implies that the product $\lambda_{t}^{i} \nu_{t}^{i}$ (for all $t$ and $i$ ) is thus always null. Including the Euler equation constraint in the planner's objective (15) yields the following objective, denoted by $J$ :

$$
J=\mathbb{E}_{0} \sum_{t=0}^{\infty} \beta^{t} \int_{i}\left(U\left(c_{t}^{i}, \hat{l}_{t}^{i}\right)-\left(\lambda_{t}^{i} U_{c}\left(c_{t}^{i}, \hat{l}_{t}^{i}\right)-\lambda_{t}^{i} \mathbb{E}_{t}\left[\left(1+r_{t+1}\right) U_{c}\left(c_{t+1}^{i}, \hat{l}_{t+1}^{i}\right)\right]\right)\right) \ell(d i) .
$$

The previous equation can be reformulated, setting $\lambda_{-1}=0$ and using the tower rule:

$$
J=\mathbb{E}_{0} \sum_{t=0}^{\infty} \beta^{t} \int_{i}\left(U\left(c_{t}^{i}, \hat{l}_{t}^{i}\right)-\left(\lambda_{t}^{i}-\lambda_{t-1}^{i}\left(1+r_{t}\right)\right) U_{c}\left(c_{t}^{i}, \hat{l}_{t}^{i}\right)\right) \ell(d i) .
$$

The Ramsey program then consists in maximizing $J$ in (16) over $\left(\left(a_{t}^{i}, c_{t}^{i}, l_{t}^{i}\right)_{i}, \phi_{t}, \tau_{t}\right)_{t \geq 0}$ subject to the relevant constraints: equations (6), (14), and (29). Using (3) to substitute for $r_{t}$ and $w_{t}$, the Lagrangian can be seen as depending only on $\left(a_{t}^{i}\right)_{i}$ and $\phi_{t}$.

The Lagrange multipliers $\lambda_{t}^{i}$ are key to understanding the planner's program. If agent's $i$ private incentives to save at date $t$ are socially optimal, then her Euler equation is not a constraint and the Lagrange multiplier is $\lambda_{t}^{i}=0$. Depending on how the planner perceives the saving distortions, these coefficients can be either positive or negative. A positive (resp. negative) Lagrange multiplier for agent $i$ reflects that the planner perceives that agent $i$ saves too little (resp. too much). We provide an example in Section E.1 of the

\footnotetext{
${ }^{10}$ We thank Albert Marcet for this suggestion.
} 
online appendix to clarify this aspect.

\subsection{Ramsey conditions and economic interpretation}

Using proper substitution, the Ramsey program (16) can be written as a maximization problem with only two sets of choice variables: the labor tax $\left(\tau_{t}\right)_{t}$ and saving choices $\left(a_{t}^{i}\right)_{t, i}$. The current section derives the planner's first-order conditions and discusses the economic trade-offs that determine the time-varying replacement rate. The derivations of these conditions can be found in Section B.1 of the online appendix.

To ease the economic interpretation of the first-order conditions, we define: ${ }^{11}$

$$
\psi_{t}^{i}=U_{c}\left(c_{t}^{i}, \hat{l}_{t}^{i}\right)-\left(\lambda_{t}^{i}-\left(1+r_{t}\right) \lambda_{t-1}^{i}\right) U_{c c}\left(c_{t}^{i}, \hat{l}_{t}^{i}\right)
$$

which will be called the marginal social valuation of liquidity for agent $i$, because it is the marginal gain for the planner of transferring resources to agent $i$ at date $t$. If agent $i$ receives one additional unit of goods today, this additional unit will have a private value proportional to $U_{c}$. The planner also has to account for the effect on the saving incentives, i.e., on the Euler equations. This additional unit therefore affects the agent's saving incentive from period $t-1$ to period $t$ and from period $t$ to period $t+1$. This effect is captured by the second term, which is proportional to $U_{c c}$.

The saving decision $a_{t}^{i}$ in history $s^{N}$ at date $t$ affects the welfare of all agents due to general equilibrium effects on capital and prices. The first-order condition of the Ramsey program related to saving choices summarizes all of these effects. It can be written as

\footnotetext{
${ }^{11}$ Unlike Chien et al. (2011) or Marcet and Marimon (2019), we do not use cumulative Lagrange multipliers to analyze the dynamics. Instead, we use the period multipliers to derive the planner's firstorder conditions. These conditions are easier to interpret and the simulation of the model relies on a smaller number of variables. See these two references for a discussion of the existence of these multipliers in such economies.
} 
follows for an unconstrained agent $i:^{12}$

$$
\begin{aligned}
& \psi_{t}^{i}=\underbrace{\beta \mathbb{E}_{t}\left[\left(1+r_{t+1}\right) \psi_{t+1}^{i}\right]}_{\text {liquidity smoothing }}+\underbrace{\beta \frac{\alpha K_{t}^{-1}}{1+\alpha \varphi} \mathbb{E}_{t}[\int_{i} \psi_{t+1}^{i} \overbrace{\left.\left(1-\tau_{t+1}\right) y_{t+1}^{i} w_{t+1} l_{t+1}^{i} 1_{e_{t+1}^{i}=e} \ell(d i)\right]}^{\text {net wage }}}_{\text {wage effect for employed }} \\
& +\underbrace{\beta \frac{\alpha K_{t}^{-1}}{1+\alpha \varphi} \mathbb{E}_{t}[\int_{i} \psi_{t+1}^{i}(1+\varphi) \overbrace{\phi_{t+1} y_{t+1}^{i} w_{t+1} l_{t+1, e}^{i} 1_{e_{t+1}^{i}=u}}^{\text {=unemp. benefits }} \ell(d i)]}_{\text {wage effect (unemp. ben.) for unemployed }} \\
& -\underbrace{\beta \frac{\alpha K_{t}^{-1}}{1+\alpha \varphi} \mathbb{E}_{t}\left[\frac{w_{t+1} L_{t+1}}{K_{t}} \int_{i}\left(\lambda_{t}^{i} U_{c}\left(c_{t+1}^{i}, \hat{l}_{t+1}^{i}\right)+\psi_{t+1}^{i} a_{t}^{i}\right) \ell(d i)\right]}_{\text {interest rate effect on smoothing and wealth }}\} .
\end{aligned}
$$

Equation (18) features the first-order condition on the liquidity allocation (i.e., saving choices) for unconstrained agents. Although it appears complicated, the equation has a straightforward interpretation. Four effects are at play. The first is a direct effect that measures the expected future value of liquidity tomorrow. In other words, this component states that liquidity value should be smoothed over time. This first part is very similar to a standard consumption Euler equation, except that it reflects "social" marginal utilities $\psi_{t}^{i}$, rather than "private" marginal utilities $U_{c}^{i}$. We refer to this first term as "liquidity smoothing". The three other components alter the pure smoothing effect and reflect the fact that the planner also takes into account the consequences of liquidity allocation on prices. More precisely, the second and third components correspond to the marginal effect of additional saving on the wage rate. This affects employed agents (second component) and unemployed (third component) agents, because UI benefits are proportional to the labor income of employed agents with the same productivity. Finally, the fourth and last component reflects the distortions through the interest rate on saving incentives.

The second first-order condition, relating to the labor tax, can be written as follows:

$$
\begin{aligned}
& \underbrace{\frac{S_{t, e}}{S_{t, u}}\left(\frac{1}{\varphi}+1-\frac{1-\alpha}{1-\tau_{t}}\right) \int_{i} \psi_{t}^{i} \frac{l_{t}^{i} y_{t}^{i}}{L_{t}} 1_{e_{t}^{i}=u} \ell(d i)}_{\text {gain of unemployment benefits for unemployed }}= \\
& \underbrace{\frac{1}{\varphi} \int_{i} \psi_{t}^{i} \frac{l_{t}^{i} y_{t}^{i}}{L_{t}} 1_{e_{t}^{i}=e^{\ell}} \ell(d i)}_{\text {cost of the tax for employed }}+\underbrace{\frac{\alpha}{\left(1-\tau_{t}\right) K_{t-1}} \int_{i}\left(\lambda_{t-1}^{i} U_{c}\left(c_{t+1}^{i}, \hat{l}_{t+1}^{i}\right)+\psi_{t+1}^{i} a_{t-1}^{i}\right)}_{\text {effect on prices, smoothing, and redistribution }} .
\end{aligned}
$$

Equation (19) determines the optimal labor tax rate by setting the marginal costs of a

\footnotetext{
${ }^{12}$ Proofs for first-order conditions can be found in Section B.1 of the online appendix.
} 
higher tax rate equal to the marginal benefits. On the left-hand side of equation (19), the marginal benefit comprises the marginal gain of tax (and UI benefit) for unemployed agents. On the right-hand side of (19), marginal costs comprise two effects. The first effect accounts for the impact of the labor tax on employed agents, taking into account the negative net effect on the labor supply (inversely proportional to the Frisch elasticity $\varphi$ ). The second one reflects the tax distortion on the interest rate and thus on saving incentives. Note that equation (19) embeds, in a compact form, the general equilibrium effect on wages, which are captured by both the Frisch elasticity of the labor supply, $\varphi$, and the concavity of the production function, $\alpha$.

\section{The aggregation and truncation theory}

This section presents our methodology. It is organized in two parts, which correspond to fixed and optimal policies, respectively. In each of the two parts, the first subsection presents the general principles that govern the methodology, either in the presence of fixed UI policy or of optimal UI policy. The second subsection then presents the steady state, while the third section discusses the introduction of aggregate shocks. Finally the fourth and last subsection presents a convergence result of our method at the steady state.

\subsection{Economy with exogenous UI policy}

\subsubsection{Aggregation of an incomplete-market model}

Our methodology consists of expressing the model in terms of groups of agents sharing the same idiosyncratic history over the last $N>0$ periods, which will be called a truncated history, rather than in terms of individual agents. The time-length $N>0$, called the truncation length, is an exogenous parameter of the method. The history over the last $N$ periods can be identified by a vector $s^{N} \in \mathcal{S}^{N}$. Considering an agent $i$ with (full) idiosyncratic history $s^{i, t}$ at date $t \geq N$, her truncated history can be represented as:

$$
s^{i, t}=\{\ldots, s_{-N-2}^{t}, s_{-N-1}^{t}, s_{-N}^{t}, \underbrace{s_{-N+1}^{t}, \ldots, s_{-1}^{t}, s_{0}^{t}}_{=s^{N}}\},
$$


where $s_{-k}^{t}$ is her idiosyncratic status (at date $t$ ) $k$ periods in the past. The resulting model will be called an aggregated model, while for the sake of clarity, we will denote the original model, the model expressed in terms of individuals of Section 2. The construction of the aggregated model proceeds in four intermediate steps.

First, we compute the measure of agents with truncated history $s^{N}$. An agent having a truncated history $\hat{s}^{N}$ at $t-1$ will have a different truncated history $s^{N}$ at $t$ depending on the realization of the idiosyncratic risk at date $t$. The probability of transitioning from $\hat{s}^{N}$ at $t-1$ to $s^{N}$ at $t$ is denoted by $\Pi_{t, \hat{s}^{N} s^{N}}\left(\right.$ with $\left.\sum_{s^{N} \in \mathcal{S}^{N}} \Pi_{t, \hat{s}^{N} s^{N}}=1\right)$ and can be inferred from the transition probabilities for unemployment and productivity:

$$
\Pi_{t, \hat{s}^{N} s^{N}}=1_{s^{N} \succeq \hat{s}^{N}} \Pi_{t, \hat{e}_{0}^{N}, e_{0}^{N}} \Pi_{\hat{y}_{0}^{N} y_{0}^{N}} \geq 0
$$

where $s_{0}^{N}=\left(e_{0}^{N}, y_{0}^{N}\right)$ and $\hat{s}_{0}^{N}=\left(\hat{e}_{0}^{N}, \hat{y}_{0}^{N}\right)$ are the current idiosyncratic states for $s^{N}$ and $\hat{s}^{N}$, respectively. We can deduce from these transition probabilities the share of agents in the population endowed with history $s^{N}$ at date $t$, which is denoted by $S_{t, s^{N}}$ :

$$
S_{t, s^{N}}=\sum_{\hat{s}^{N} \in \mathcal{S}^{N}} S_{t-1, \hat{s}^{N}} \Pi_{t, \hat{s}^{N} s^{N}}
$$

where the initial shares $\left(S_{-1, s^{N}}\right)_{s^{N} \in \mathcal{S}^{N}}$, with $\sum_{s^{N} \in \mathcal{S}^{N}} S_{-1, s^{N}}=1$, are given.

Second, the aggregated model involves computing consumption, saving, and labor choices for groups of agents with the same truncated history $s^{N}$. In the original model with the aggregate shocks of Section 2, the equilibrium allocation can be characterized by a set of policy rules (for saving, consumption, and labor supply) and Lagrange multipliers (for the borrowing limit), defined over all idiosyncratic and aggregate histories:

$$
a_{t}\left(s^{t}, z^{t}\right), c_{t}\left(s^{t}, z^{t}\right), l_{t}\left(s^{t}, z^{t}\right), \nu_{t}\left(s^{t}, z^{t}\right), t \geq 0, s^{t} \in \mathcal{S}^{t}, z^{t} \in \mathcal{Z}^{t}
$$

These quantities have to be computed for the aggregated model. Consider one of these variables, denoted by $X$. We will assign to a truncated history $s^{N}$ the quantity $X_{t, s^{N}}$, which is defined as the following average:

$$
X_{t, s^{N}}=\frac{1}{S_{t, s^{N}}} \sum_{s^{t} \in \mathcal{S}^{t} \mid\left(s_{t-N+1}^{t}, \ldots, s_{t}^{t}\right)=s^{N}} X_{t}\left(s^{t}, z^{t}\right) \mu_{t}\left(s^{t}\right),
$$

where we recall that $\mu_{t}\left(s^{t}\right)$ is the measure of agents with history $s^{t}$. With the definition 
(23), $c_{t, s^{N}}, a_{t, s^{N}}, l_{t, s^{N}}$, and $\nu_{t, s^{N}}$ are respectively the average consumption, the end-ofperiod saving, the labor supply and the credit-constraint Lagrange multiplier among agents having at date $t$ the truncated history $s^{N}$.

Third, we have to compute aggregate beginning-of-period wealth. Because agents switch histories from one period to another, the per capita beginning-of-period wealth for history $s^{N}$ at date $t$ is derived from period- $(t-1)$ end-of-period wealth and from the transition across histories. Indeed, it consists of the wealth of all agents having history $s^{N}$ in period $t$ and any other possible history in $t-1$. Formally, the beginning-of-period wealth $\tilde{a}_{t, s^{N}}$ for truncated history $s^{N}$ is:

$$
\tilde{a}_{t, s^{N}}=\sum_{\hat{s}^{N} \in \mathcal{S}^{N}} \frac{S_{t-1, \hat{s}^{N}}}{S_{t, s^{N}}} \Pi_{t, \hat{s}^{N}, s^{N}} a_{t-1, \hat{s}^{N}} .
$$

Fourth, the method involves the aggregation of Euler equations. As the marginal utility is not linear in consumption in the general case, the marginal utility of consumption aggregation is different from the aggregation of marginal utilities. If we denote the latter aggregation by $u_{t, s^{N}}^{\prime}$ for truncated history $s^{N}$ at date $t$, we formally have: $u^{\prime}\left(c_{t, s^{N}}\right) \neq u_{t, s^{N}}^{\prime}$, unless there is no consumption heterogeneity within truncated histories $s^{N}$. However, we can compute the set of parameters $\left(\xi_{t, s^{N}}\right)_{t, s^{N}}$ that guarantees that Euler equations hold with aggregate consumption levels. As a consequence, the $\xi$ s can be seen as the relevant summary of within-truncated-history heterogeneity for the dynamics of the aggregated model and will be henceforth called residual heterogeneity parameters. Indeed, we show in the numerical investigation of Section 5 that the $\xi$ s efficiently capture this within-history heterogeneity in a parsimonious way, even with a short truncation, such as $N=2 .{ }^{13}$

The $\xi$ s parameters only apply to consumption Euler equations and not to the labor Euler equations. This is a result from the GHH utility function, which implies that the labor supply Euler equation is linear in current productivity (see equation (9)). Hence, there is no truncation wedge when aggregating the labor supply equation. However, it would be present with a more general utility function, and correcting coefficients would be needed for both Euler equations. Though slightly more involved, it is noteworthy that this would not impair the construction of the aggregated model. See Section E.3 of the

\footnotetext{
${ }^{13}$ These parameters are related to the aggregation procedure presented in Werning (2015). The difference is that Werning (2015) captures the heterogeneity through a change in the discount factor, whereas we capture with these $\xi$ s. This is only important to derive Ramsey allocations.
} 
online appendix for further details.

The four previous steps can now be used to compute the aggregated model starting from the equations (6)-(9) characterizing the equilibrium in the original model. The aggregation yields the following set of equations:

$$
\begin{aligned}
c_{t, s^{N}}+a_{t, s^{N}} & \leq\left(1+r_{t}\right) \tilde{a}_{t, s^{N}} \\
& +\left(\left(1-\tau_{t}\right) 1_{e_{0}^{N}=e} l_{t, s^{N}}+\phi_{t} 1_{e_{0}^{N}=u} l_{t, s^{N}, e}\right) y_{0}^{N} w_{t} \\
\xi_{t, s^{N}} U_{c}\left(c_{t, s^{N}}, \hat{l}_{t, s^{N}}\right) & =\beta \mathbb{E}_{t}\left(1+r_{t+1}\right) \sum_{\tilde{s}^{N} \in \mathcal{S}^{N}} \Pi_{t+1, s^{N}, \tilde{s}^{N}} \xi_{t+1, \tilde{s}^{N}} U_{c}\left(c_{t+1, \tilde{s}^{N}}, \hat{l}_{t+1, \tilde{s}^{N}}\right)+\nu_{t, s^{N}} \\
l_{t, s^{N}}^{\frac{1}{\varphi}} & =\chi\left(1-\tau_{t}\right) w_{t} y_{0}^{N} \\
\hat{l}_{t, s^{N}}^{i} & =l_{t, s^{N}} 1_{e_{0}^{N}=e}+\zeta_{y_{0}^{N}} 1_{e_{0}^{N}=u} .
\end{aligned}
$$

The budget constraint (25) is the aggregation of individual budget constraints (6) using equations (23) and (24). The same holds for the Euler labor equation (27) that comes from individual Euler equation (9). Finally, the aggregated Euler equation (25) for consumption relies on the parameters $\left(\xi_{t, s^{N}}\right)$ that enable the aggregation of individual Euler equations (8). The system (25)-(28) is an exact aggregation of the original model with aggregate shocks in terms of truncated idiosyncratic histories. It characterizes the dynamics of the aggregated variables $c_{t, s^{N}}, a_{t, s^{N}}, l_{t, s^{N}}$, and $\nu_{t, s^{N}}$ without involving any approximation.

Finally, market clearing conditions can also be expressed in terms of aggregated variables. For capital and labor we have:

$$
K_{t}=\sum_{s^{N} \in \mathcal{S}^{N}} S_{t, s^{N}} a_{t, s^{N}}, L_{t}=\sum_{s^{N} \in \mathcal{S}^{N}} S_{t, s^{N}} y_{s^{N}} l_{t, s^{N}}
$$

The equilibrium in the aggregated model is characterized by the set of equations (25)-(29). The aggregation mechanism involves no simplifying assumption and, for any truncation length $N>0$, it is always possible to compute the allocation of an aggregated model based on the allocation of an original model. However, we aim at doing the opposite: taking advantage of the finite-state space properties of the aggregated model to simulate it and gain knowledge about the original model. We now explain how to make the aggregated model operational. 


\subsubsection{Economy with exogenous UI policy and no aggregate shock}

The first step to make the aggregated model operational involves computing the $\xi$ s at the steady state. Consider an original Bewley model associated to an exogenous UI policy $(\phi, \tau)$. The outcome of this original Bewley model is denoted by $\left(r, w,\left(a_{s^{\infty}}, c_{s^{\infty}}, l_{s^{\infty}}, \nu_{s^{\infty}}\right)_{s^{\infty} \in \mathcal{S}^{\infty}}\right)$, where $(r, w)$ are prices and $\left(a_{s^{\infty}}, c_{s^{\infty}}, l_{s^{\infty}}, \nu_{s^{\infty}}\right)_{s^{\infty} \in \mathcal{S}^{\infty}}$ is the allocation denoted using the sequential representation. We formally define the probability space associated to the set $\mathcal{S}^{\infty}$ of infinite idiosyncratic histories in Section B.3.1 of the online appendix. The following proposition presents the computation of the $\xi s$.

Proposition 1 (Constructing the $\xi \mathbf{s}$ ) Consider a truncation length $N>0$, and an original Bewley model, $\left(r, w,\left(a_{s^{\infty}}, c_{s^{\infty}}, l_{s^{\infty}}, \nu_{s^{\infty}}\right)_{s^{\infty} \in \mathcal{S}^{\infty}}\right)$ for a UI policy $(\phi, \tau)$. The steadystate value of the residual heterogeneity parameters $\left(\xi_{s^{N}}\right)_{s^{N} \in \mathcal{S}^{N}}$ can be computed such that the following conditions hold:

1. the tuple $\left(S_{s^{N}}, a_{s^{N}}, c_{s^{N}}, l_{s^{N}}, \nu_{s^{N}}\right)$, consisting of the steady-state truncation history sizes, savings, consumption levels, labor supplies, and credit-constraint Lagrange multipliers in the aggregated model, is computed using the allocation $\left(a_{s^{\infty}}, c_{s} \infty, l_{s} \infty\right.$, $\left.\nu_{s^{\infty}}\right)_{s^{\infty} \in \mathcal{S}^{\infty}}$ of the original Bewley model and using the idiosyncratic transition probabilities $\left(\Pi_{y y^{\prime}}\right)$ and $\left(\Pi_{e e^{\prime}}\right)$ with equations (21), (22), and (23);

2. the set of credit constraints histories, $\mathcal{C}_{N}$, is determined based on the aggregated credit-constraint Lagrange multipliers: $\mathcal{C}_{N}=\left\{s^{N} \in \mathcal{S}^{N}: \nu_{s^{N}} \geq \bar{\nu}_{N}\right\}$, where $\bar{\nu}_{N}$ is an exogenously set threshold;

3. the Euler equations (26) for consumption hold at the steady state in the aggregated model.

The proof is provided in Section C.2.1 of the online appendix, and it can be summarized as follows. At the steady state, given an exogenous UI policy, we can solve the original model and compute the policy functions and the stationary wealth distribution. From these elements, we can compute with equation (23) the aggregated quantities. Note that transition probabilities for truncated histories are deduced from idiosyncratic transition probabilities $\left(\Pi_{y y^{\prime}}\right)$ and $\left(\Pi_{t, e e^{\prime}}\right)$ with equation $(21)$, while the population of agents with the given truncated history is given by (22). This completes Point 1 of Proposition 1. 
The set of credit-constrained truncated histories, denoted by $\mathcal{C}_{N}$, is determined using credit-constraint Lagrange multipliers for truncated histories, $\nu_{s^{N}}$. Credit-constrained truncated histories are those with the highest Lagrange multipliers $\nu_{s^{N}}$. Formally, all truncated histories featuring a Lagrange multiplier $\nu_{s^{N}}$ above a threshold $\bar{\nu}_{N}$ will be credit-constrained. The threshold is chosen such that the mass of credit-constrained agents in both the original and the aggregated models is similar. This sets Point 2 of Proposition 1.

Once we have computed the consumption, labor supply, and Lagrange multipliers for truncated histories (i.e., the triplet $\left.\left(c_{s^{N}}, l_{s^{N}}, \nu_{s^{N}}\right)\right)$, the $\xi \mathrm{s}$ can be computed by inverting Euler equations (26) in the aggregated model. This computation actually pins down to simple linear algebra. We provide in equation (83) of Section C.2.1 in the online appendix a closed-form expression for the $\xi$ s. Point 3 therefore holds by construction of the $\xi \mathrm{s}$.

\subsubsection{Economy with exogenous UI policy and aggregate shocks}

To make our aggregation procedure in the presence of aggregate shocks operational, we need to impose the following assumptions.

Assumption A We assume that in the presence of aggregate shocks:

1. the residual heterogeneity parameters $\left(\xi_{s^{N}}\right)_{s^{N}}$ remain constant and equal to their steady-state values;

2. the set of credit-constrained histories, $\mathcal{C}_{N}$, is time-invariant and determined at the steady state.

The first item of Assumption A means that the model features within-history heterogeneity, but that this heterogeneity is not time-varying, as the preference parameters $\left(\xi_{s^{N}}\right)_{s^{N}}$, determined at the steady state, remain constant in the presence of aggregate shocks. We thus assume the model dynamics is computed while considering that withintruncated-history heterogeneity is not time-varying. This assumption is in the same vein as the assumption that the within-bin heterogeneity is uniform in the histogram approach of Reiter (2009). 
The second item of Assumption A states that if a history $s^{N} \in \mathcal{S}^{N}$ is credit-constrained at the steady state, it also remains credit-constrained in the dynamic version of the model. This restriction is imposed by perturbation methods and is not specific to our construction. ${ }^{14}$ Despite this assumption, the number of credit-constrained households can be time-varying, since the measure of agents having a given truncated history is time-varying.

Assumption A enables us to formally characterize the so-called truncated model. It is defined by the set of equations (25)-(28), together with: (i) the additional assumptions that $\xi_{t, s^{N}}=\xi_{s^{N}}$ for all $t$; and (ii) the fact that Euler equations (25) only hold for the non-credit-constrained histories determined at the steady state. The definition can be stated as follows.

Definition 2 (Truncated equilibrium) A truncated equilibrium is a collection of individual variables $\left(c_{t, s^{N}}, l_{t, s^{N}}, a_{t, s^{N}}, \nu_{t, s^{N}}\right)_{t \geq N-1}^{s^{N} \in \mathcal{S}^{N}}$, of aggregate quantities $\left(K_{t}, L_{t}, Y_{t}\right)_{t \geq N-1}$, of price processes $\left(w_{t}, r_{t}\right)_{t \geq N-1}$, and of UI policy $\left(\tau_{t}, \phi_{t}\right)_{t \geq N-1}$, such that, for an initial wealth distribution $\left(a_{N-2, s^{N}}\right)_{s^{N} \in \mathcal{S}^{N}}$, and for initial values of capital stock $K_{N-2}=$ $\sum_{s^{N} \in \mathcal{S}^{N}} a_{N-2, s^{N}}$, and of the aggregate shock $z_{N-2}$, we have:

1. $\left(\xi_{s^{N}}\right)_{s^{N} \in \mathcal{S}^{N}}$ are the steady-state preference parameters of the aggregated model;

2. individual strategies $\left(c_{t, s^{N}}, l_{t, s^{N}}, a_{t, s^{N}}, \nu_{t, s^{N}}\right)_{t \geq N-1, s^{N} \in \mathcal{S}^{N}}$ solve the agent's optimization program in equations (25)-(10);

3. financial, labor, and goods markets clear: for any $t \geq N-1$, equations (29) hold;

4. the UI budget is balanced: equation (4) holds for all $t \geq N-1$;

5. factor prices $\left(w_{t}, r_{t}\right)_{t \geq N-1}$ are consistent with the firm's program (3).

We now explain how we can use Proposition 1 and Assumption A to simulate the truncated model in the presence of aggregate shocks.

Algorithm 1 Consider a truncation length $N>0$ and a UI policy $(\phi, \tau)$. The algorithm for simulating the truncated model with aggregate shocks can be stated as follows.

\footnotetext{
${ }^{14}$ In particular, it could be relaxed by using history-specific penalty functions to model (possibly large) aggregate shocks. We leave this development for future work.
} 
1. Solve for the original Bewley model given UI policy $(\phi, \tau)$. This gives individual allocations, aggregate quantities and prices.

2. Construct the aggregated model at the steady state following Proposition 1.

(a) Compute aggregate quantities $\left(S_{s^{N}}, c_{s^{N}}, l_{s^{N}}, a_{s^{N}}, \nu_{s^{N}}\right)_{s^{N}}$, using individual allocations and equation (23).

(b) Characterize the set of credit-constrained truncated histories $\mathcal{C}_{N}$.

(c) Compute $\left(\xi_{s^{N}}\right)_{s^{N}}$ by inverting the Euler equations (26) - see the closed-form expression (83) in Section C.2.1 of the online appendix.

3. Construct the truncated model (defined by equations (25)-(29)) using Assumption A.

4. The truncated model can then be simulated in the presence of aggregate shocks using perturbation methods.

The perturbation methods of Step 4 can be conducted using a standard software such as Dynare. The steady state around which the perturbation is conducted is computed as the aggregation of the underlying original Bewley model. Remark also that, by construction, the original and truncated models feature the same steady-state aggregate quantities (such as GDP, capital, total labor supply, etc.) and the same prices.

Our truncation method makes it possible to rely on off-the-shelf and well-established computational software to solve for heterogeneous-agent models in the presence of aggregate shocks. The key advantage of our method is that, once the $\xi \mathrm{s}$ have been computed at the steady state, the truncated model features limited heterogeneity and therefore lends itself to a computation through perturbation methods, explained in Algorithm 1 (see Section C.1 of the online appendix for the list of equations). ${ }^{15}$

\subsubsection{A steady-state convergence result}

We provide a convergence result for the truncation method, when the history length $N$ becomes increasingly long. We need to state a number of additional assumptions so as to

\footnotetext{
${ }^{15}$ The truncated model can also be shown to be constructed from an explicit partial-insurance mechanism, where individual agents can only insure against idiosyncratic shocks occurring $N+1$ periods ago. This alternative construction is presented in Section A of the online appendix using an island metaphor (see Lucas, 1975, 1990). One of the interests of this alternative construction is to prove that the dynamic of the truncated model is well-defined and can be written in a recursive form.
} 
guarantee the existence of the steady-state equilibrium for the original model. We here follow Açikgöz (2018), which applies in our GHH setup for a given UI policy.

Assumption B We make the following set of assumptions:

1. the credit-constraint is set to zero: $\underline{a}=0$;

2. steady-state wage and interest rates verify: $w>0$ and $0<\beta(1+r)<1$;

3. steady-state labor tax verifies: $\left(\left(1+\frac{1}{\varphi}\right) \frac{S_{e}}{S_{u}}+1\right)^{-1}<\tau<1$.

4. utility function verifies:

$$
\liminf _{c \rightarrow \infty}-\frac{u^{\prime \prime}(c)}{u^{\prime}(c)}=0
$$

5. the steady-state transition matrices $\left(\Pi_{y y^{\prime}}\right)$ for productivity and $\left(\Pi_{e e^{\prime}}\right)$ for unemployment are irreducible and verify $\Pi_{\underline{y}, \underline{y}}>0, \Pi_{e e}>0$ and $\Pi_{u u}>0$, where $\underline{y}$ is the lowest productivity level.

Let us comment on the different points of Assumption B. Point 1 sets the credit constraint $\underline{a}$ to zero, as in Açikgöz (2018). Note that such a choice is always possible, up to renormalization of the income process. Point 2 is similar to Assumption 2 in Açikgöz (2018), and it avoids discussing irrelevant cases. Point 3 is specific to our framework and has two objectives. First, it ensures that the income of employed or unemployed agents cannot be nonpositive and thus simply extends $w>0$ in our case. Second, through the condition $\left(\left(1+\frac{1}{\varphi}\right) \frac{S_{e}}{S_{u}}+1\right)^{-1}<\tau$, it implies that the "aggregate consumption" of unemployed agents, equal to $c-\chi^{-1} \frac{\zeta_{y}^{1+1 / \varphi}}{1+1 / \varphi}$, is positive at the steady state. The "aggregate consumption" of employed agents can be shown to be also positive whenever $\tau<1$. Together with the properties of the utility function, it guarantees that the agent's program in the original Bewley economy is well-defined and can be solved by continuous policy functions (defined on the product space of beginning-of-period asset holdings and idiosyncratic risk). Points 1 to 4 imply that the agent's state space can be chosen to be compact. Point 4 , which is similar to Assumption 3 of Açikgöz (2018), holds for the standard CRRA utility function (independently of the choice of the elasticity of substitution) that is most often used in quantitative applications - and that we use in Section 5. Point 5, with previous points, is sufficient to prove the existence of a unique stationary distribution - and also that agents' 
aggregate asset demand diverges to infinity when $\beta(1+r) \rightarrow 1$. Assumption B ultimately implies the existence of a stationary recursive equilibrium for a given UI policy.

We can now state our result regarding the convergence of allocations in the aggregated model at the steady state, as well as the convergence of the $\xi$ s parameters. The proof can be found in Section B.3 of the online appendix.

Proposition 2 (Convergence of allocations) Consider an original Bewley model, whose prices and allocations are denoted by $\left(r, w,\left(a_{s^{\infty}}, c_{s^{\infty}}, l_{s^{\infty}}, \nu_{s^{\infty}}\right)_{s^{\infty} \in \mathcal{S}^{\infty}}\right)$ for a given UI policy $(\phi, \tau)$. For any truncation length $N>0$, let $\left(r, w,\left(c_{s^{N}}, a_{s^{N}}, l_{s^{N}}, \nu_{s^{N}}, \xi_{s^{N}}\right)_{s^{N} \in \mathcal{S}^{N}}\right)$ be the prices and allocations of the aggregated model constructed using Proposition 1. We have the following results:

1. $\left(r, w,\left(c_{s^{N}}, l_{s^{N}}, a_{s^{N}}, \nu_{s^{N}}\right)_{s^{N} \in \mathcal{S}^{N}}\right) \longrightarrow_{N}\left(r, w,\left(a_{s^{\infty}}, c_{s^{\infty}}, l_{s^{\infty}}, \nu_{s^{\infty}}\right)_{s^{\infty} \in \mathcal{S}^{\infty}}\right)$, almost surely;

2. $\left(\xi_{s^{N}}\right)_{s^{N} \in \mathcal{S}^{N}} \longrightarrow_{N}(1)_{s^{\infty} \in \mathcal{S}^{\infty}}$, almost surely.

Proposition 2 states that when the truncation length increases, the allocation of the aggregated economy converges to the allocation of the underlying original Bewley model, while the $\xi_{\mathrm{s}}$ converge to one. In the proposition, we used the fact that prices are the same in the original Bewley model and in the aggregate one (for all truncation lengths $N)$. The intuition for the result of Proposition 2 is as follows. As $N$ increases, the agents' shared history becomes longer and the first period with a potentially different idiosyncratic status becomes more distant. This means that as $N$ increases, the agents assigned to a given truncated history become more "similar" to each other and the allocation of the aggregated model converges to the allocation of the original Bewley model. Furthermore, since the heterogeneity within each truncated history vanishes, the $\xi$ s have an increasingly smaller role to play and converge to 1 for large $N$.

\subsection{Economy with optimal policy}

\subsubsection{Ramsey program}

We use the truncated model to compute optimal Ramsey policies in a heterogeneous-agent model with aggregate shocks. There are two main gains of computing Ramsey allocations in the truncated model rather than aggregating first-order conditions of the original Ramsey 
program. First, we can prove the convergence results of Ramsey allocations in the truncated model toward the true allocation, when the truncation length becomes increasingly long. Second, this strategy ensures the numerical stability of dynamic simulations, as we solve for Ramsey policies in a well-defined model (see Section B.2 of the online appendix).

Following Section 3, the truncated Ramsey problem can be written as:

$$
\max _{\left(\left(a_{t, s^{N}}, c_{t, s^{N}}, l_{t, s^{N}}, \hat{l}_{t, s^{N}}\right)_{s \in \mathcal{S}^{N}}, \phi_{t}, \tau_{t}, r_{t}, w_{t}\right)_{t \geq 0}} \mathbb{E}_{0}\left[\sum_{t=0}^{\infty} \beta^{t} \sum_{s^{N} \in \mathcal{S}^{N}} S_{t, s^{N}} \xi_{s^{N}} U\left(c_{t, s^{N}}, \hat{l}_{t, s^{N}}\right)\right],
$$

subject to: (i) the budget constraints (25), (ii) the Euler equations (26)-(27), (iii) the market clearing constraints (29), and (iv) the factor prices (3), (v) the UI budget balance (14).

As in the original program, we define the social value of liquidity $\Psi_{t, s^{N}}$ as:

$$
\Psi_{t, s^{N}}=\xi_{s^{N}} U_{c}\left(c_{t, s^{N}}, \hat{l}_{t, s^{N}}\right)-\left(\lambda_{t, s^{N}}-\left(1+r_{t}\right) \tilde{\lambda}_{t, s^{N}}\right) \xi_{s^{N}} U_{c c}\left(c_{t, s^{N}}, \hat{l}_{t, s^{N}}\right)
$$

where $\tilde{\lambda}_{t, s^{N}}$ is the aggregation of previous-period Lagrange multipliers $\lambda_{t-1, \tilde{s}^{N}}$ and is defined similarly to $\tilde{a}_{t, s^{N}}$ in equation (24):

$$
\tilde{\lambda}_{t, s^{N}}=\sum_{\tilde{s}^{N} \in \mathcal{S}^{N}} \frac{S_{t-1, \tilde{s}^{N}}}{S_{t, s^{N}}} \Pi_{t, \tilde{s}^{N} s^{N}} \lambda_{t-1, \tilde{s}^{N}}
$$

The parameter $\Psi_{t, s^{N}}$ is the exact parallel for histories of $\psi_{t}^{i}$ defined in (17) for individuals. Similarly to the original case, two first-order conditions need to be computed. With respect to savings, we obtain for unconstrained histories $s^{N} \in \mathcal{S}^{N} \backslash \mathcal{C}_{N}$ :

$$
\begin{gathered}
\times\left\{\mathbb{E}_{t}\left[\sum_{s^{N} \in \mathcal{S}^{N}} \Psi_{t+1, \tilde{s}^{N}} S_{t+1, \tilde{s}^{N}} w_{t+1}\left(\left(1-\tau_{t+1}\right) l_{t+1, \tilde{s}^{N}} 1_{\tilde{e}_{0}^{N}=e}+(1+\varphi) \phi_{t+1} l_{t+1, \tilde{s}^{N}, e} 1_{\tilde{e}_{0}^{N}=u}\right) \tilde{y}_{0}^{N}\right]\right. \\
\left.-\sum_{\tilde{s}^{N} \in \mathcal{S}^{N}} \mathbb{E}_{t}\left[\frac{w_{t+1} L_{t+1}}{K_{t}} S_{t+1, \tilde{s}^{N}}\left(\tilde{\lambda}_{t+1, \tilde{s}^{N}} \xi_{\tilde{s}^{N}} U_{c}\left(c_{t+1, s^{N}}, \hat{l}_{t+1, s^{N}}\right)+\Psi_{t+1, \tilde{s}^{N}} \tilde{a}_{t+1, \tilde{s}^{N}}\right)\right]\right\}
\end{gathered}
$$

while gather first-order relative to the labor tax can be written as follows:

$$
\frac{\alpha}{\left(1-\tau_{t}\right) K_{t-1}} \sum_{\tilde{s}^{N} \in \mathcal{S}^{N}} S_{t, \tilde{s}^{N}}\left(\tilde{\lambda}_{t, \tilde{s}^{N}} \xi_{\tilde{s}^{N}} U_{c, t, s^{N}}+\Psi_{t, \tilde{s}^{N}} \tilde{a}_{t, \tilde{s}^{N}}\right)+\frac{1}{\varphi_{\tilde{s}^{N} \in \mathcal{S}^{N}}} S_{t, \tilde{s}^{N}} \Psi_{t, \tilde{s}^{N}} \frac{l_{t, \tilde{s}^{N}}}{L_{t}} \tilde{y}_{0}^{N} 1_{\tilde{e}_{0}^{N}=e} .
$$


It can be observed that first-order conditions (33) and (34) of the truncated Ramsey program are very similar to (18) and (19) for the original Ramsey program.

\subsubsection{Economy with optimal policy and no aggregate shock}

In the Ramsey program of Section 4.2.1, the planner takes the $\xi \mathrm{s}$ as given and solves, at the steady state, for the optimal corresponding allocation $\left(c_{s^{N}}, l_{s^{N}}, a_{s^{N}}\right)_{s^{N} \in \mathcal{S}^{N}}$ and the optimal UI policy $(\phi, \tau)$. This first step is easy to implement and yields an allocation that is consistent with the truncated representation. In the following algorithm, we impose an additional consistency constraint. This constraint requires the $\xi$ s (capturing residual heterogeneity) to correspond to the allocation of the original Bewley model given the optimal UI policy. More precisely, once the optimal UI policy $(\phi, \tau)$ has been computed, we can solve for the original Bewley model corresponding to this policy, and derive the residual heterogeneity parameters $\tilde{\xi} s$ implied by this Bewley allocation. While these $\tilde{\xi} s$ may differ from the $\xi$ s used to compute the optimal policy, our second consistency requirement imposes these parameters to be the same: $\xi_{\mathrm{s}}=\tilde{\xi}_{\mathrm{s}}$. This constraint on the $\xi$ s should be seen as a refinement of our method to take advantage of the maximum information from the original Bewley model. The following algorithm ensures that the consistency requirement over the $\xi$ s holds by looking for a fixed point over the $\xi$ s until the $\xi$ s used to compute the optimal UI policy are the same as the $\xi$ s constructed using the optimal UI policy and Proposition 1.

Algorithm 2 The algorithm for computing the steady-state Ramsey allocation can be stated as follows.

1. Choose a truncation length $N$.

2. Set an initial value for the UI policy $(\phi, \tau)$.

3. Solve for the allocation of the original Bewley model given the policy $(\phi, \tau)$.

4. Construct the corresponding aggregated model as follows:

(a) Compute aggregated allocation $\left(c_{s^{N}}, l_{s^{N}}, a_{s^{N}}\right)_{s^{N}}$ using the allocation of the original Bewley model and equation (23). 
(b) Inverse Euler equations (26) to compute $\left(\xi_{s^{N}}\right)_{s^{N}}$. See the closed-form expression (83) in Section C.2.1 for a direct computation of the online appendix.

(c) Use equation (33) at the steady state to compute $\left(\Psi_{s^{N}}\right)_{s^{N}}$ and Lagrange multipliers $\left(\lambda_{s^{N}}\right)_{s^{N}}$. See the closed-form expressions (84) and (85) in Section C.2.2 of the online appendix for a direct computation.

5. Compute both sides of optimality condition (19). If equality does not hold (up to a precision criterion), update the UI policy, and iterate starting at Step 3.

6. Finally, increase $N$ and repeat Steps 2-5 until the optimal UI policy does not change.

This algorithm delivers a policy $(\phi, \tau)$ such that: (i) the aggregated allocation $\left(c_{s^{N}}, l_{s^{N}}, a_{s^{N}}\right)_{s^{N}}$ and the $\xi s$ are both derived from an allocation $\left(a_{s^{\infty}}, c_{s^{\infty}}, l_{s^{\infty}}, \nu_{s^{\infty}}\right)_{s^{\infty} \in \mathcal{S}^{\infty}}$ of the original Bewley model given the policy $(\phi, \tau)$; (ii) the policy $(\phi, \tau)$ is optimal in the truncated model defined by $\left(c_{s^{N}}, l_{s^{N}}, a_{s^{N}}\right)_{s^{N}}$ and the $\xi s$. In other words, we find the optimal policy in the truncated model with the $\xi s$ that are consistent with the original Bewley model (given the optimal policy).

Algorithm 2 has two main advantages. First, the aggregated model is, by construction, consistent with the corresponding original Bewley model. In particular, aggregate quantities (capital, consumption, labor) and prices are exactly the same in the steady-state Ramsey model and in the corresponding original Bewley model. Second, our algorithm requires the Bewley model to exist for each UI policy (because of Step 3). The Ramsey planner must therefore select an existing competitive equilibrium and perturbation methods - used to compute the model with aggregate shocks - cannot be run around non-existing steady-state equilibria (which could be the case if we did not impose aggregated allocation $\left(c_{s^{N}}, l_{s^{N}}, a_{s^{N}}\right)_{s^{N}}$ to be the aggregation of an original Bewley allocation $\left(a_{s^{\infty}}, c_{s^{\infty}}, l_{s^{\infty}}\right)_{\left.s^{\infty} \in \mathcal{S}^{\infty}\right) .}$

\subsubsection{Economy with optimal policy and aggregate shocks}

Once the steady state of the model has been computed, the dynamics of the Ramsey model - provided in Section C.1 of the online appendix - can be solved by perturbation techniques, as there are a finite number of equations. This implies that the (truncated) 
wealth distribution and the distribution of Lagrange multipliers are used as state variables. As shown in the numerical analysis of Section 5, the planner's instruments depend on these two distributions. As in the case of exogenous policy, the simulation can rely on existing software (e.g., Dynare).

Algorithm 3 The algorithm for solving the Ramsey program in the presence of aggregate shocks can be stated as follows.

1. Use Algorithm 2 to compute the steady-state Ramsey allocation and set an acceptable truncation length.

2. Set the system characterizing the Ramsey solution: equations (25)-(29) for the competitive equilibrium and equations (33) and (34) for Ramsey FOCs.

3. Simulate the previous system using perturbation methods around the steady-state allocation of Step 1.

4. Check that IRFs and second-order moments are not affected by changing $N$.

\subsubsection{A steady-state convergence result}

We provide a second convergence result for the truncation method, still for a steady-state economy. For the sake of simplicity, we will denote an original (steady-state) Ramsey equilibrium by $\left(\phi, \tau, r, w,\left(a_{s^{\infty}}, c_{s^{\infty}}, l_{s^{\infty}}, \nu_{s^{\infty}}\right)_{s^{\infty} \in \mathcal{S}^{\infty}}\right)$ where $\phi$ is the replacement rate, $\tau$, the associated labor tax, $(r, w)$ are prices, and $\left(a_{s^{\infty}}, c_{s^{\infty}}, l_{s^{\infty}}, \nu_{s^{\infty}}\right)_{s^{\infty} \in \mathcal{S} \infty}$, the allocation of the original Bewley program corresponding to UI policy $(\phi, \tau)$. The replacement rate $\phi$ and the labor $\operatorname{tax} \tau$ are connected though the UI policy budget equation (14). Similarly, we denote by $\left(\phi_{N}, \tau_{N}, r_{N}, w_{N},\left(c_{s^{N}}, l_{s^{N}}, a_{s^{N}}, \nu_{s^{N}}, \xi_{s^{N}}\right)_{s^{N} \in \mathcal{S}^{N}}\right)$ the Ramsey equilibrium for an aggregated Ramsey model with truncation length $N>0$, where the UI policy and prices can in general depend on the truncation length. We assume that Assumption B also holds here.

The following result characterizes the convergence of Ramsey allocations in the aggregated model at the steady state, when the truncation length increases. Each Ramsey allocation (for a given truncation length) can be thought of as being computed with Algorithm 3. Indeed, assuming full convergence of this algorithm will imply that Assumptions 
1 to 3 of Proposition 3 hold (or are straightforward to check). The proof can be found in Section B.4 of the online appendix.

Proposition 3 (Convergence of allocations) We consider a sequence of Ramsey equilibria $\left(\phi_{N}, \tau_{N}, r_{N}, w_{N},\left(a_{s^{N}}, c_{s^{N}}, l_{s^{N}}, \nu_{s^{N}}, \xi_{s^{N}}\right)_{s^{N} \in \mathcal{S}^{N}}\right)_{N \geq 0}$, such that:

1. for all $N$, there exists an original Bewley allocation $\left(a_{s^{\infty}, N}, c_{s^{\infty}, N}, l_{s^{\infty}, N}, \nu_{s^{\infty}, N}\right)_{s^{\infty} \in \mathcal{S}^{\infty}}$ such that the aggregation following equation (23) of $\left(c_{s^{\infty}, N}, l_{s^{\infty}, N}, a_{s^{\infty}, N}, \nu_{s^{\infty}, N}\right)_{s^{\infty} \in \mathcal{S}^{\infty}}$ yields the aggregated allocation $\left(a_{s^{N}}, c_{s^{N}}, l_{s^{N}}, \nu_{s^{N}}\right)_{s^{N} \in \mathcal{S}^{N}}$;

2. for all $N$, the first-order equations characterizing the aggregated Ramsey equilibrium, (25)-(29) and (33)-(34), hold for $\left(\phi_{N}, \tau_{N}, r_{N}, w_{N},\left(a_{s^{N}}, c_{s^{N}}, l_{s^{N}}, \nu_{s^{N}}, \xi_{s^{N}}\right)_{s^{N} \in \mathcal{S}^{N}}\right)$;

3. there exist $\left(\left(1+\frac{1}{\varphi}\right) \frac{S_{e}}{S_{u}}+1\right)^{-1}<\underline{\tau}<\bar{\tau}<1$, such that for all $N: \underline{\tau} \leq \tau_{N} \leq \bar{\tau}$;

4. there exists a unique solution denoted $\left(\phi^{*}, \tau^{*}, r^{*}, w^{*},\left(a_{s^{\infty}}^{*}, c_{s^{\infty}}^{*}, l_{s^{\infty}}^{*}, \nu_{s^{\infty}}^{*}\right)_{s^{\infty} \in \mathcal{S}^{\infty}}\right)$, solving the original Ramsey program of Section 3 (characterized by equations (6)-(14) and (18)-(19)).

Then, we have the following convergence results:

1. the UI policy $\left(\phi_{N}, \tau_{N}\right)$ converges with $N$ to $\left(\phi^{*}, \tau^{*}\right)$;

2. prices $\left(r_{N}, w_{N}\right)$ converge with $N$ to $\left(r^{*}, w^{*}\right)$;

3. allocations $\left(a_{s^{N}}, c_{s^{N}}, l_{s^{N}}, \nu_{s^{N}}\right)_{s^{N} \in \mathcal{S}^{N}}$ converge almost surely with $N$ to the allocation $\left(a_{s^{\infty}}^{*}, c_{s^{\infty}}^{*}, l_{s^{\infty}}^{*}, \nu_{s^{\infty}}^{*}\right)_{s^{\infty} \in \mathcal{S}^{\infty}}$;

4. $\left(\xi_{s^{N}}\right)_{s^{N} \in \mathcal{S}^{N}} \longrightarrow{ }_{N}(1)_{s^{\infty} \in \mathcal{S}^{\infty}}$, almost surely.

Proposition 3 states that this sequence of aggregated Ramsey allocations converges toward the unique original Ramsey allocation $\left(\phi^{*}, \tau^{*}, r^{*}, w^{*},\left(a_{s^{\infty}}^{*}, c_{s^{\infty}}^{*}, l_{s^{\infty}}^{*}, \nu_{s^{\infty}}^{*}\right)_{s^{\infty} \in \mathcal{S}^{\infty}}\right)$. The $\xi$ s are also shown to converge to 1 . This convergence result completes the convergence result for the original Bewley allocation of Proposition 2.

Two points are worth noting. First, Proposition 3 assumes that the UI tax lies in a compact set. This avoids a situation where $\tau_{N} \rightarrow 1$ or $\tau_{N} \rightarrow\left(\left(1+\frac{1}{\varphi}\right) \frac{S_{e}}{S_{u}}+1\right)^{-1}$, implying that the income or the consumption of some agents could be null at the limit. In that 
case, Points 2 and 3 of Assumption B would not hold any more. Second, we require the existence of a unique solution to the original Ramsey program of Section 3. In the absence of such a condition, we can only obtain the convergence for a subsequence. This weaker result, related to the possibility of multiple equilibria, is stated in Proposition 5 of Section B.4 of the online appendix.

\section{Dynamics of the optimal replacement rate}

We now turn to the quantitative analysis. We provide the calibration in Section 5.1. We then provide steady-state results in Section 5.2. The model dynamics with a fixed replacement rate is presented in Section 5.3. The optimal policy at the steady state is discussed in Section 5.4, and the optimal policy with aggregate shocks, which is the purpose of the paper, is presented in Section 5.5. The comparison of the truncation method with other methods, together with additional accuracy checks, are provided in Section 6 .

\subsection{The calibration}

\subsubsection{Preferences}

The period is a quarter. The discount factor is $\beta=0.99$. The period utility function is $\log \left(c-\chi^{-1} \frac{l^{1+1 / \varphi}}{1+1 / \varphi}\right)$. The Frisch elasticity of labor supply is set to $\varphi=0.5$, which is the value recommended by Chetty et al. (2011) for the intensive margin in heterogeneous agent models. The scaling parameter is set to $\chi=0.04$, which implies normalizing the aggregate labor supply, defined in (11), to $1 / 3$.

Unemployed workers cannot choose their labor supply. Their utility function is $\log \left(c-\chi^{-1} \frac{\zeta_{y}^{1+1 / \varphi}}{1+1 / \varphi}\right)$, also with $\chi=0.04$ and $\varphi=0.5$. We recall that $\zeta_{y}$ is the exogenous labor supply for home production for a worker with productivity $y$. For agents to be worse-off when unemployed than employed, $\zeta_{y}$ is set to the steady-state labor supply of a worker with productivity $y$.

\subsubsection{Technology and TFP shock}

The production function is Cobb-Douglas: $Y=Z K^{\alpha} L^{1-\alpha}$. The capital share is set to $\alpha=36 \%$ and the depreciation rate is $\delta=2.5 \%$, as in Krueger et al. (2018) among others. 
The TFP process is a standard process, with $Z_{t}=\exp \left(z_{t}\right)$ and $z_{t}=\rho_{z} z_{t-1}+\varepsilon_{t}^{z}$, where $\varepsilon_{t}^{z} \stackrel{\text { iid }}{\sim} \mathcal{N}\left(0, \sigma_{z}^{2}\right)$. We use the standard values $\rho_{z}=0.95$ and $\sigma_{z}=0.31 \%$ to obtain a deviation of the TFP shock $z_{t}$ equal to $1 \%$ at the quarterly frequency (see e.g., Den Haan, 2010).

\subsubsection{Idiosyncratic risk}

Unemployment risk. For the unemployment risk, we follow Shimer (2003) and assume that the job-separation rate is constant over the business cycle, while the job-finding rate is time-varying and procyclical. We set $\Pi_{e u}^{S S}=4.87 \%$ for the average job-separation rate and $\Pi_{u e}^{S S}=78.6 \%$ for the average job-finding rate. The standard deviation of the job-finding rate is set to $6 \%$, based on US estimates (see Abeille-Becker and Clerc, 2013, or Challe and Ragot, 2016). As the standard deviation of $z_{t}$ is $1 \%$, we assume that the job-finding rate is defined as $\Pi_{t, u e}=\Pi_{u e}^{S S}+\sigma_{u e} z_{t}$, with $\sigma_{u e}=6$.

\begin{tabular}{cll}
\hline Parameter & \multicolumn{1}{c}{ Description } & Value \\
\hline$\beta$ & Discount factor & 0.99 \\
$\alpha$ & Capital share & 0.36 \\
$\delta$ & Depreciation rate & 0.025 \\
$\Pi_{u e}^{S S}$ & Average job-finding rate & $78.6 \%$ \\
$\bar{a}$ & Credit limit & 0.0 \\
$\Pi_{e u}^{S S}$ & Average job-separation rate & $4.87 \%$ \\
$S_{u}^{S S}$ & Steady-state unemployment rate & $5.83 \%$ \\
$\rho_{z}$ & Autocorrelation TFP & 0.95 \\
$\sigma_{z}$ & Standard deviation TFP shock & $0.31 \%$ \\
$\sigma_{u e}$ & Cov. job find. rate with TFP & 6.0 \\
$\rho_{y}$ & Autocorrelation idio. income & 0.992 \\
$\sigma_{y}$ & Standard dev. idio. income & $6.60 \%$ \\
$\chi$ & Scaling param. labor supply & 0.04 \\
$\varphi$ & Frisch elasticity labor supply & 0.5 \\
\hline
\end{tabular}

Table 1: Parameter values in the baseline calibration. See text for descriptions and targets.

Idiosyncratic productivity risk. Idiosyncratic productivity risk is a key ingredient for the model to generate a realistic earning and wealth distribution. We calibrate a productivity process $\log y_{t}=\rho_{y} \log y_{t-1}+\varepsilon_{t}^{y}$, with $\varepsilon_{t}^{y} \stackrel{\text { iid }}{\sim} \mathcal{N}\left(0, \sigma_{y}^{2}\right)$. As we are considering a model with endogenous labor supply, there is a difference between the earning process and the productivity process. With the GHH utility function, the labor supply is $l_{y}=$ 
$(w(1-\tau) \chi y)^{\varphi}$. The log of labor income $y l_{y}$ is thus related to $(1+\varphi) \log y$ and accordingly depends on the value chosen for the Frisch elasticity. We calibrate the process $y$ such that the persistence and variance of the labor income $y l_{y}$ match the estimated values of Krueger et al. (2018). They estimate a process with a persistent and transitory shock on productivity. Following Boppart et al. (2018), we use persistent shocks and consider transitory shocks as measurement errors. Using a Frisch elasticity of 0.5 , we compute a quarterly persistence of $\rho_{y}=0.9923$ and a standard deviation of $\sigma_{y}=6.60 \%$, which generate, for the log of earnings, an annual persistence of 0.9695 and a variance of $\frac{3.84 \%}{1-0.9695^{2}} .16$ The Rouwenhorst (1995) procedure is used to discretize the productivity process into 7 idiosyncratic states with a constant transition matrix. As agents can be either employed or unemployed, each agent can be in one of the $14=7 \times 2$ idiosyncratic states. Table 1 provides a summary of the model parameters.

\subsection{Fixed UI policy at the steady state}

We simulate a Bewley model with a constant and exogenous replacement rate $\phi$. The computational details are provided in Section D.1 of the online appendix. In Table 2, we report the wealth distribution generated by the model and compare it to the empirical distribution. We compute a number of standard statistics - listed in the first column including the quartiles, the Gini coefficient, and the 90-95 and 95-100 intercentiles.

The empirical wealth distribution, reported in the second and third columns of Table 2, comes from two sources, the PSID for the year 2006 and the SCF for the year 2007. The fourth and fifth columns report the wealth distribution generated by our model with two different values for the exogenous replacement rate $\phi$, set either to $50 \%$ (column 4 ) or to $42 \%$ (column 5 ). The former value of $\phi=50 \%$ corresponds to the standard value used in the literature for the US, as in Krueger et al. (2018) among others. The latter value of $\phi=42 \%$ corresponds to the optimal steady-state replacement rate that we compute below (see Section 5.4), and is here provided to observe the effect of a change in the replacement rate on the steady-state wealth distribution.

\footnotetext{
${ }^{16}$ We follow the procedure of Footnote 19 in Krueger et al. (2018). The quarterly persistence $\rho_{y}$ is such that $\rho_{y}^{4}=0.9695$ equals the annual persistence. The variance of the log of labor income $y l_{y}$ (at the quarterly frequency) is the same as the variance at the annual frequency, so: $(1+\varphi) \frac{\sigma_{y}^{2}}{1-\rho_{y}^{2}}=\frac{0.0384}{1-0.9695^{2}}$.
} 
Overall, the distribution of wealth generated by the model is quite similar for the two replacement rate values and is close to the data. In particular, the model does a good job in matching the wealth distribution with a high Gini of 0.70 . The concentration of wealth at the top of the distribution is higher in the data than in the model. It is known that additional model features must be introduced to match the high wealth inequality in the US, such as heterogeneous discount rates, as in Krusell and Smith (1998), or entrepreneurship, as in Quadrini (1999).

\begin{tabular}{ccccc}
\hline & \multicolumn{2}{c}{ Data } & \multicolumn{2}{c}{ Models } \\
\hline Wealth statistics & PSID, 06 & SCF, 07 & $\phi=50 \%$ & $\phi=42 \%$ \\
\hline Q1 (minimum) & -0.9 & -0.2 & 0.2 & 0.3 \\
Q2 & 0.8 & 1.2 & 1.4 & 1.8 \\
Q3 (median) & 4.4 & 4.6 & 6.2 & 6.4 \\
Q4 & 13.0 & 11.9 & 19.5 & 18.7 \\
Q5 (maximum) & 82.7 & 82.5 & 71.6 & 68.7 \\
90-95 intercentile & 13.7 & 11.1 & 16.9 & 16.9 \\
95-100 intercentile & 36.5 & 36.4 & 32.9 & 32.8 \\
Gini & 0.77 & 0.78 & 0.70 & 0.69 \\
\hline
\end{tabular}

Table 2: Wealth distribution in the data and in the model.

Steady-state effect of a change in the replacement rate. To better understand the effect of the replacement rate, Table 3 compares some steady-state statistics for two economies, featuring the replacement rate of either $42 \%$ or $50 \%$.

\begin{tabular}{ccccc}
\hline & $\phi$ & $K$ & $C$ & $c^{u} / c^{e}$ \\
\hline Economy (1) & $42.0 \%$ & 13.1428 & 0.8382 & 0.9823 \\
Economy (2) & $50.0 \%$ & 13.1304 & 0.8380 & 0.9847 \\
\hline Variation (\%) & $8.0 \%^{\dagger}$ & $-0.1 \%$ & $-0.02 \%$ & $-0.24 \%$ \\
\hline
\end{tabular}

Note: See the text for definitions of the variables. $\dagger$ indicates an absolute variation, while the variation by default is a relative variation.

Table 3: Implications of a variation in the steady-state replacement rate

The first two rows correspond to the two economies, while the third row corresponds to the relative change for the variable of interest, reported in the columns. It can be observed that an increase in the replacement rate of $8 \%$ decreases the capital stock, $K$, 
and aggregate consumption, $C$, by a small amount. Due to the higher replacement rate, unemployed agents are better insured by the UI scheme and agents thus express a smaller demand for self-insurance. They therefore save less, which diminishes total savings and capital. This also decreases the consumption inequality $c^{u} / c^{e}$ between unemployed and employed workers. The higher replacement rate also means a higher labor tax that deters labor supply and agents' labor earnings, which has a negative impact on savings and consumption.

\subsection{Fixed UI policy with aggregate shocks}

We now compute the dynamics of the model with a fixed replacement rate, set to $\phi=42 \%$, and with aggregate shocks affecting both TFP and the job-finding rate. The dynamics of the model is solved using the truncation procedure with a truncation length $N=2$. We use these steady-state allocations to compute the $\xi$ s, which ensure that the truncated model has the same truncated wealth distribution as the steady-state Bewley model. Importantly, this low value of $N$ is enough to replicate the dynamics of the model, thanks to the $\xi$ s. The model captures relevant heterogeneity in productivity levels and transitions, as discussed in Section 4. We gather in Section 6 the discussion of the accuracy of the method. For the sake of clarity, we summarize the equations of the model in Section C.1 of the online appendix.

We compare the behaviors of the incomplete-market economy (IM) and the completemarket one (CM) for a fixed replacement rate, set exogenously to $42 \%$. The CM model features a unique agent and one Euler equation. All parameters are otherwise the same. Results correspond to Economies (1) and (2) of Table 4, respectively. Economies (3) and (4) are discussed in Section 5.5 and are provided in the same table to ease the comparison. Table 4 reports, for each economy, the mean and the normalized standard deviation (i.e., the standard deviation divided by the mean) of the main aggregate variables: output $Y$, aggregate consumption $C$, total labor $L$, and the replacement rate $\phi$. The autocorrelations and correlations for consumption and output are also given. Each economy is simulated over 10,000 periods.

In the incomplete-market economy (Economy (1)), the capital stock is higher than in the complete market one (Economy (2)) since the agents form some precautionary 


\begin{tabular}{llcccc}
\hline Replacement rate & \multicolumn{2}{c}{ Exogenous rate } & \multicolumn{2}{c}{ Optimal rule } \\
\hline Methods & & IM & CM & IM & CM \\
\hline Economies & & $(1)$ & $(2)$ & $(3)$ & $(4)$ \\
\hline$Y$ & Mean & 1.17 & 1.08 & 1.17 & 1.08 \\
& Std/mean (\%) & 2.18 & 2.10 & 2.64 & 2.81 \\
$C$ & Mean & 0.84 & 0.80 & 0.84 & 0.80 \\
& Std/mean (\%) & 1.76 & 1.73 & 2.26 & 2.35 \\
$L$ & Mean & 0.30 & 0.30 & 0.30 & 0.30 \\
& Std/mean (\%) & 1.03 & 0.70 & 1.67 & 1.47 \\
$K$ & Mean & 13.14 & 11.09 & 13.14 & 11.09 \\
& Std/mean (\%) & 2.09 & 2.18 & 2.29 & 2.93 \\
$\phi$ & Mean & 42.0 & 42.0 & 42.0 & 42.0 \\
& Std/mean (\%) & 0.0 & 0.0 & 23.0 & 23.0 \\
$\operatorname{corr}\left(C, C_{-1}\right)$ & $(\%)$ & 99.23 & 99.66 & 98.74 & 99.67 \\
$\operatorname{corr}\left(Y, Y_{-1}\right)$ & & 97.64 & 98.19 & 97.41 & 98.23 \\
$\operatorname{corr}(C, Y)$ & & 95.87 & 94.59 & 97.19 & 95.26 \\
$\operatorname{corr}(Y, \Phi)$ & & 0.0 & 0.0 & -97.18 & -96.43 \\
\hline
\end{tabular}

Table 4: Moments of the simulated models for different specifications and different computational techniques.

savings against the idiosyncratic risk. The correlation between consumption and output being higher in incomplete-market economies, the incomplete-market economy is also more volatile that the complete-market one, which is a standard outcome of those models.

\subsection{Optimal UI policy at the steady state}

The optimal steady-state replacement rate is computed using the algorithm described in Section 4.2.2. This algorithm yields an optimal steady-state replacement rate of $\phi=42 \%$, which we used in the simulations of Section 5.3. This optimal replacement rate is obtained for $N=2$, which implies that we simulate $14^{2}=196$ histories. We have checked that we obtain the same optimal replacement rate, $\phi=42 \%$, for $N=3$, with 2,744 histories. In this last case, the computations are slower.

The trade-offs faced by the planner have already been presented in the discussion of Table 3. An increase in the replacement rate reduces inequality and capital accumulation. Increasing the replacement rate is a distorting tool to provide insurance against the unemployment risk. It also disincentivizes savings, as it decreases labor supply and 
consumption.

These replacement rates have been computed using the algorithm described in Section 4.2.2. In particular, the calculations involved the $\xi s$ that are consistent with the original Bewley allocation. In order to investigate the sensitivity of our algorithm to the consistency constraint on the $\xi \mathrm{s}$, we have also computed the steady optimal replacement rate while imposing $\xi s=1$. We find that the optimal replacement rate is then equal to $40 \%$, instead of $42 \%$ with the consistent $\xi \mathrm{s}$. The optimal policy is in our case quite robust to a variation in the $\xi$ s. For the simulations below, we use the $\xi s$ that are consistent with the underlying Bewley model, as the dynamics appears to be more sensitive than the steady state to the choice of the $\xi s$ (see Section 6.1).

\subsection{Optimal UI policy with aggregate shocks}

We now compute the dynamics of the model using perturbation methods, about which more details are provided in Section $\mathrm{C}$ of the online appendix. The dynamic properties of the replacement rate, solved in the truncated economy, are reported in Economy (3) of Table $4 .{ }^{17}$ The average replacement rate is $42 \%$ and its standard deviation is $23 \%$. The comparison between Economies (1) (with a fixed replacement rate of 42\%) and (3) shows the effect of a time-varying replacement rate. It can be seen that the replacement rate is countercyclical, since $\operatorname{corr}(Y, \phi)=-0.93<0$. It increases in recessions and decreases in booms. As the replacement rate is countercyclical, labor supply, aggregate consumption, and output are more procyclical in Economy (3) than in Economy (1). ${ }^{18}$

We investigate the dynamics of the optimal replacement rate in the truncated economy to plug it into the complete-market economy. We simulate the model for 10,000 periods and regress the replacement rate $\phi_{t}$ on several moments of aggregate variables. We find that the following (and rather complex) rule has a very high $R^{2}=0.99999$ :

(35) $\phi_{t}=\left(1-a_{1}^{\phi}-a_{2}^{\phi}\right) \phi^{s s}+a_{1}^{\phi} \phi_{t-1}+a_{2}^{\phi} \phi_{t-2}+a_{0}^{\varepsilon} \varepsilon_{t}^{z}+a_{1}^{\varepsilon} \varepsilon_{t-1}^{z}+a_{2}^{\varepsilon} \varepsilon_{t-2}^{z}+a^{K}\left(K_{t-1}-\bar{K}\right)+\varepsilon_{t}^{\phi}$,

\footnotetext{
${ }^{17}$ The simulation of the economy with the optimal replacement rate implies 7 variables, $(c, a, \tilde{a}, \lambda, \tilde{\lambda}, l, S)$, for the $14^{2}=196$ histories. As a consequence, there are roughly 1500 equations in our perturbation procedure. Using Dynare, it takes around 2 minutes to simulate the model with a standard laptop.

${ }^{18}$ In the literature, results about the cyclicality of the replacement rate are mixed. Mitman and Rabinovich (2015) find a procyclical replacement rate, whereas Landais et al. (2018a,b) find a countercyclical one. These papers study economies without capital, but with a much more detailed model of the labor market. We instead investigate the implication of an endogenous capital stock, but with a simpler labor market structure.
} 
with $\left(\phi^{s s}, a_{1}^{\phi}, a_{2}^{\phi}, a_{0}^{\varepsilon}, a_{1}^{\varepsilon}, a_{2}^{\varepsilon}, a^{K}\right)=(0.420,-23.907,0.011,0.602,0.482,0.371,-0.117) .{ }^{19} \mathrm{We}$ can now plug this estimated rule into the complete-market economy to simulate it. The results correspond to Economy (4) in Table 4.

One can check that a countercyclical replacement rate increases the volatility in a complete-market economy compared to the case where the replacement rate is constant (Economies (2) vs. (4)). However, the incomplete-market economy is now less volatile than the complete-market one (Economies (3) vs. (4)), as there is more insurance for the unemployment risk in recessions, which reduces time-varying precautionary saving. The planner indeed wants to avoid a sharp decrease in the consumption of agents with the highest marginal utilities, which would strongly reduce aggregate welfare in recessions.

\section{Method comparisons, accuracy of the truncation method, and additional tests}

\subsection{Method comparison}

We here assess the accuracy of the truncation method used in the previous section. We compare the truncated method with two other methods. The first is the method developed by Rios-Rull (1999), Reiter (2009), and Young (2010) among others, which we call the Reiter method for brevity. This method uses a histogram representation and perturbation methods to solve for the model. ${ }^{20}$ The method is known to provide accurate results, when compared to the global method of Krusell and Smith (1998), as shown in Boppart et al. (2018) or in Auclert et al. (2019). We also compare the results of the truncation approach to the recent method of Boppart et al. (2018) - hereafter BKM - for TFP shocks.

Second-order moments. We simulate the same economy as in Section 5.3, with the same parameters. To follow the comparison method of Boppart et al. (2018), we focus on TFP shocks only $\left(\sigma_{u e}=0\right)$. The results are reported in Table 5 (Economies (1) to (6)).

\footnotetext{
${ }^{19}$ We also estimated a simpler rule. We regress the replacement rate $\phi_{t}$ on the technology shock $z$ and on the first, second, and third-order moments of the wealth distribution, to see whether moments of the wealth distribution can be sufficient statistics. We find a lower $R^{2}$ of 0.73 . It appears that a rich time structure is necessary to reproduce the dynamics of the optimal replacement rate.

${ }^{20}$ The Reiter method can be used with bases other than histograms, such as in Winberry (2018) or Bayer et al. (2019).
} 


\begin{tabular}{lcccccc}
\hline Methods & RA & BKM & Reiter & $\begin{array}{c}\text { Trunc. } \\
(N=2)\end{array}$ & $\begin{array}{c}\text { Trunc. } \\
(N=3)\end{array}$ & $\begin{array}{c}\text { Trunc. } \\
(\xi=1)\end{array}$ \\
\hline Economies & \multicolumn{1}{c}{$(1)$} & \multicolumn{1}{c}{$(2)$} & \multicolumn{1}{c}{$(3)$} & \multicolumn{1}{c}{$(4)$} & \multicolumn{1}{c}{$(5)$} & $(6)$ \\
\hline$Y \quad$ Mean & 1.08 & 1.17 & 1.17 & 1.17 & 1.17 & 1.17 \\
$\quad$ Std/mean (\%) & 2.10 & 1.75 & 1.77 & 1.78 & 1.78 & 1.74 \\
$C \quad$ Mean & 0.80 & 0.84 & 0.84 & 0.84 & 0.84 & 0.84 \\
$\quad$ Std/mean (\%) & 1.73 & 1.45 & 1.45 & 1.45 & 1.45 & 1.46 \\
$L \quad$ Mean & 0.30 & 0.30 & 0.30 & 0.30 & 0.30 & 0.30 \\
$\quad$ Std/mean (\%) & 0.70 & 0.58 & 0.59 & 0.59 & 0.59 & 0.58 \\
$K \quad$ Mean & 11.09 & 13.14 & 13.14 & 13.14 & 13.14 & 13.14 \\
$\quad$ Std/mean (\%) & 2.18 & 1.61 & 1.62 & 1.68 & 1.68 & 1.54 \\
$\operatorname{corr}\left(C, C_{-1}\right)(\%)$ & 99.66 & 99.13 & 99.13 & 99.14 & 99.14 & 99.07 \\
$\operatorname{corr}(Y, Y-1)$ & 98.19 & 97.48 & 97.49 & 97.54 & 97.54 & 97.43 \\
$\operatorname{corr}(C, Y)$ & 94.59 & 96.17 & 96.21 & 96.29 & 96.23 & 96.24 \\
\hline
\end{tabular}

Table 5: Moments of the simulated model for different computational techniques

The first column describes the computed statistics (using simulations with 10,000 periods). Subsequent columns correspond to the representative-agent (RA) economy (Economy (1)), the Boppart et al. (2018) methodology (Economy (2)), the Reiter method (Economy (3)), the truncation method with the baseline methodology with $N=2$ (Economy (4)), the truncation method with $N=3$ (Economy (5)), and the truncation method with $N=2$ where we impose $\xi_{\mathrm{s}}=1$ (Economy (6)). In this last experiment, the Euler equations do not hold with equality and feature residuals, which are history-specific. This experiment allows us to quantify the role of the $\xi$ s in reproducing the dynamics.

First, the three methods (Reiter, BKM, truncation in Economies (2)-(4)) yield very similar results - and are very different from the RA economy (Economy (1)). For instance, the normalized standard deviation of GDP is between $1.75 \%$ and $1.78 \%$ for the three methods, whereas it is $2.10 \%$ in the RA case. The three methods indeed rely on a first-order approximation of the dynamics after aggregates shocks. This shows that the $\xi s$ efficiently capture the within-history heterogeneity and that a time-varying between-history is enough to reproduce well the dynamics. Second, solving the model with $N=2$ or $N=3$ does not significantly change the results (the changes are smaller than the precision criterion we use), as the $\xi s$ are computed in each case to match the wealth distribution. Third, imposing $\xi=1$ reduces the accuracy of the truncation method, but the impact is modest, which is reassuring regarding the robustness of the truncation method. 
Comparisons of IRFs. Figure 1 plots the IRFs for the main variables of interest after a TFP shock of $1 \%$. These IRFs are the ones generated by the Reiter (Economy (3)) and the baseline truncated economy with $N=2$ (Economy (4)) in Table 5.
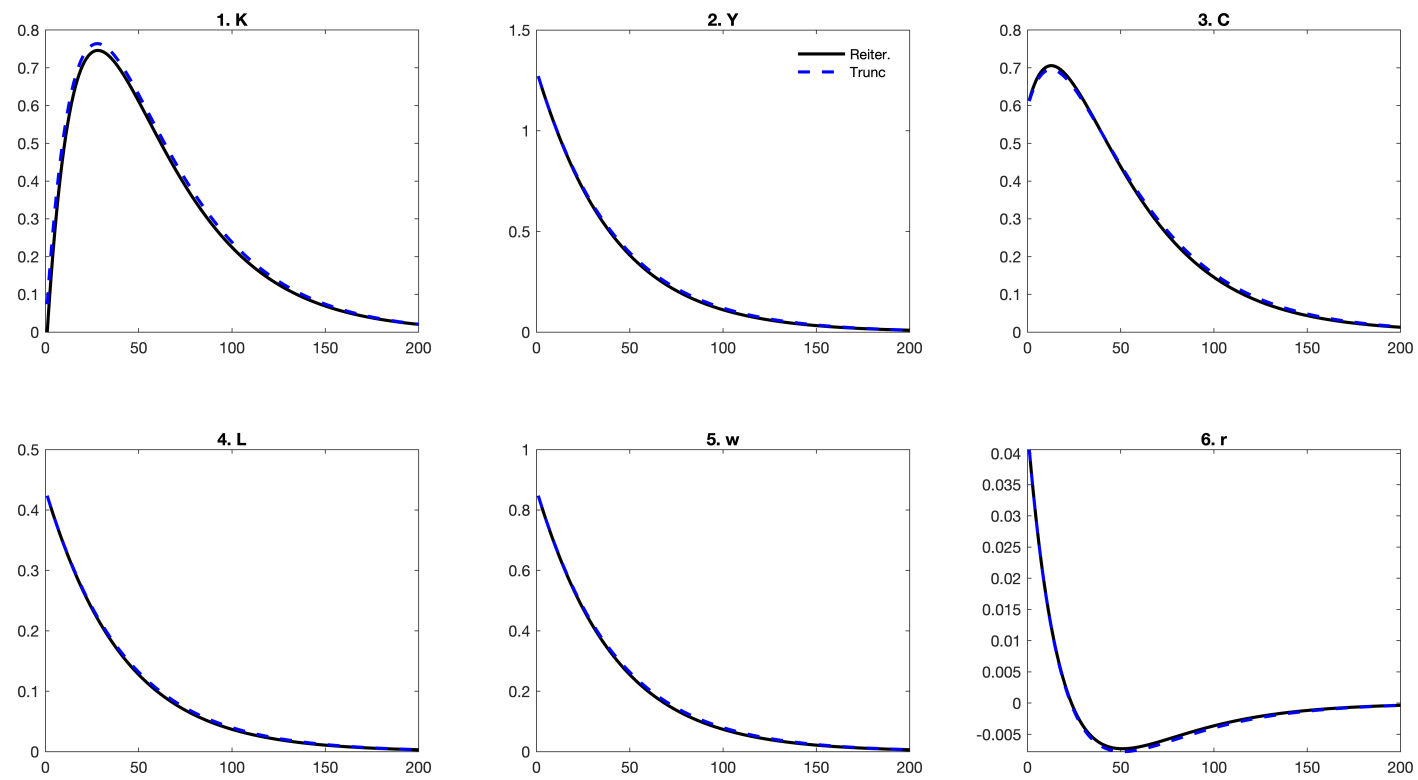

Figure 1: Comparisons of IRFs for the main variables after a technology shock of $1 \%$. The black line is the Reiter method. The blue dashed line is the truncation method.

The two sets of IRFs are extremely similar, confirming the results of Table 5, stating that the Reiter and truncation methods yield very close quantitative outcomes. We also provide additional statistics, such as correlations, in Section D.2 of the online appendix.

\subsection{Alternative rules}

To check for the optimality of the time-varying rule of equation (35), we also simulate the model using the Reiter method and implement two variations of the rule and quantify their impact on aggregate welfare. The aggregate welfare is computed using an intertemporal utilitarian welfare criterion. We report the increase in consumption $\Delta^{c}$ for all agents in all periods (agents have thus a period utility $\left.u\left(\left(1+\Delta^{c}\right) c_{t}^{i}-\chi^{-1} \frac{\left(l_{t}^{i}\right)^{1+1 / \varphi}}{1+1 / \varphi}\right)\right)$ for the aggregate welfare to be identical in economies with modified rules and in the benchmark economy (with the rule (35)). The results are collected in Table 6. Economy (1) corresponds to the benchmark economy, while Economies (2) and (3) correspond to rule variations.

The first variation is a procyclical replacement rate, where we change the signs of $a_{0}^{\varepsilon}$, $a_{1}^{\varepsilon}, a_{2}^{\varepsilon}$, and $a^{K}$. This is Economy (2) in Table 6 . We find that aggregate welfare decreases 


\begin{tabular}{llcccc}
\hline Economies & & & $(1)$ & $(2)$ & $(3)$ \\
\hline & & & Benchmark & Procyclical & High variance \\
\hline$Y$ & Mean & 1.17 & 1.17 & 1.17 & 1.17 \\
& Std/mean (\%) & 2.64 & 2.71 & 2.13 & 2.98 \\
$C$ & Mean & 0.84 & 0.84 & 0.84 & 0.84 \\
& Std/mean (\%) & 2.26 & 2.27 & 1.24 & 2.51 \\
$L$ & Mean & 0.30 & 0.30 & 0.30 & 0.30 \\
& Std/mean (\%) & 1.67 & 1.70 & 0.32 & 2.02 \\
$K$ & Mean & 13.14 & 13.14 & 13.14 & 13.14 \\
& Std/mean (\%) & 2.29 & 2.45 & 1.52 & 2.66 \\
$\phi$ & Mean & 42.0 & 42.0 & 42.0 & 42.0 \\
& Std/mean (\%) & 23.0 & 23.0 & 26.0 & 34.0 \\
$\operatorname{corr}\left(C, C_{-1}\right)$ & $(\%)$ & 98.74 & 99.18 & 98.72 & 99.19 \\
$\operatorname{corr}\left(Y, Y_{-1}\right)$ & & 97.41 & 97.52 & 97.66 & 97.50 \\
$\operatorname{corr}(C, Y)$ & & 97.19 & 95.30 & 97.29 & 94.84 \\
$\operatorname{corr}(Y, \Phi)$ & & -97.18 & -96.65 & 92.05 & -96.30 \\
Equiv. cons. & & & - & 1.72 & 1.75 \\
increase $\Delta^{c}$ & & & & & \\
\hline
\end{tabular}

Table 6: Impact of different rules for the replacement rate.

with this modified rule: a number of agents now have a lower consumption level and a higher marginal utility in recessions. The second variation is a rule featuring the same cyclicality as the original rule, but a higher variance - such that the standard deviation of $\phi_{t}$ becomes $34 \%$ instead of $23 \%$. This variation corresponds to Economy (3) in Table 6. Welfare is again decreasing because the replacement rate falls considerably in good times. This indeed leads to a decrease in the consumption of unemployed agents.

Both rules imply a decrease in welfare compared to the benchmark economy, around $1.7 \%$ of consumption equivalent. We can thus be confident in the rule's optimality - and thereby in the outcomes of the Ramsey program solved with the truncation method. These modified rules also allow to better understand the role of the optimal rule in the business cycle, which attempts to stabilize the consumption of low-utility agents.

\subsection{Convergence properties of the $\xi \mathrm{s}$}

For a given $N$, we compute $\left(\xi_{s^{N}}\right)_{s^{N} \in \mathcal{S}^{N}}$ as explained in Section 4 and then deduce the normalized standard deviation $\operatorname{std}(\xi)$ across histories. The values $\operatorname{std}(\xi)=0.27$ for $N=2$ and it diminishes to $\operatorname{std}(\xi)=0.22$ for $N=3$. The normalized standard deviation $\operatorname{std}(\xi)$ 
decreases with $N$, but at a slow rate. ${ }^{21}$ As the number of idiosyncratic states is high, equal to 14 , the number of histories grows rapidly with $N$, which computationally limits the maximal truncation length. As explained above, our choice of $N$ is not based on the minimization of the normalized standard deviations of $\xi$.

\subsection{Accuracy tests}

Finally, we perform two other standard accuracy tests. We start with Euler Equation error tests (see Den Haan and Marcet, 1994, Aruoba et al. 2006, and Den Haan, 2010) on both the steady-state model and the model with aggregate shocks. They consist in computing the absolute errors (on a base-10 log scale) implied by the exact Euler equations using the simulated allocations. The results (including mean, standard deviation, and distribution of errors) are reported in Table 7 . A value of -3 for this error, which is approximately the value found in the three cases, means a $\$ 1$ mistake for $\$ 1,000$ of consumption. This is generally considered as being an acceptable error, as discussed in Faraglia et al. (2019) for instance.

\begin{tabular}{crrc}
\hline & Static & \multicolumn{2}{c}{ Dynamic } \\
& Bewley & Exo. $\phi$ & Endo. $\phi$ \\
\hline Average & -3.91 & -4.08 & -3.95 \\
Std. dev. & 0.63 & 0.57 & 0.70 \\
\hline$[-2, \infty)$ & 0.00 & 0.00 & 0.00 \\
{$[-3,-2)$} & 1.47 & 3.01 & 5.11 \\
{$[-4,-3)$} & 96.57 & 32.14 & 53.93 \\
{$[-5,-4)$} & 1.78 & 59.72 & 33.73 \\
{$[-6,-5)$} & 0.06 & 4.59 & 5.94 \\
$(-\infty,-6)$ & 0.10 & 0.53 & 1.29 \\
\hline
\end{tabular}

Table 7: Euler equation errors

For the steady-state model with $\phi=42 \%$, the results can be found in the second column of Table 7, labeled "Bewley". The average error amounts to -3.91 , which lies within the admissible range. The results for the model with aggregate shocks are reported in the third and fourth columns of Table 7. The third column, labelled "Exo. $\phi$ ", corresponds to

\footnotetext{
${ }^{21}$ More generally, simulating different economies, we find that these normalized standard deviations decrease faster when the persistence of the idiosyncratic shocks is low, not necessarily when the number of idiosyncratic states is small.
} 
an exogenous replacement rate $\phi$, set to $42 \%$. The mean absolute log error is -4.08 , which is also completely acceptable. The fourth column, labelled "Exo. $\phi$ ", reports the result for an endogenously optimal replacement rate $\phi$. The average error is equal to -3.95 , which is slightly higher than for the exogenous $\phi$ but completely remains in the acceptable range. This accuracy test shows that the perturbation approach we use, which ignores some potential non-linearities, has a limited quantitative impact.

Our second set of tests concerns the assumption that credit-constrained histories remain constant in the dynamics (second point of Assumption A). The test is run as follows. We simulate the model - with both exogenous and endogenous replacement rates - over 10, 000 periods. We then check that the saving decision of each unconstrained history remains above the credit limit in the simulations. Conversely, we also check that credit-constrained histories remain constrained in the dynamics by checking the sign of the Euler inequality. We find that savings remain positive for all unconstrained histories and that all Euler inequalities have the correct sign for constrained histories. Finally, as noted by Den Haan (2010), these accuracy tests are not sufficient to characterize the overall goodness-of-fit of a simulation method. We consider the results of Table 4 as the main evidence of the truncation method's relevance.

\section{Conclusion}

This paper presents a truncation representation of incomplete insurance market models with aggregate shocks. We construct a finite-dimensional state-space representation, which can be simulated with aggregate shocks, and for which optimal Ramsey policies can be derived. We apply the theory to characterize optimal time-varying unemployment benefits when the economy is hit by aggregate shocks. The model simulation uses perturbation methods, which considerably eases implementation. Such methods, however, rely on small aggregate shocks around a well-defined steady state. They are less relevant for models with large macroeconomic shocks, for which additional developments are needed, using penalty functions or global methods for instance.

The theory could obviously be used for a number of other applications. The underlying model could be generalized to examine relevant frictions on the goods, labor, or financial 
markets, such as limited participation on financial markets or nominal frictions. In addition, the planner could use other tools to reduce distortions, such as a whole set of fiscal or monetary policy instruments. We are currently working on the design of optimal fiscal-monetary policies in these environments (see LeGrand et al., 2021). The simplicity of the implementation could contribute to a more systematic integration of redistributive effects in the design of economic policies. 


\section{References}

Abeille-Becker, C. And P. ClerC (2013): "The Cyclical Behavior of the Unemployment, Job Finding and Separation Rates," Revue Économique, 64, 519-526, (in French).

Ábrahám, A., J. Brogueira de Sousa, R. Marimon, and L. Mayr (2019): "On the Design of a European Unemployment Insurance System," Working Paper, European University Institute.

AçIKGÖz, O. (2018): "On the Existence and Uniqueness of Stationary Equilibrium in Bewley Economies with Production," Journal of Economic Theory, 173, 18-55.

Açikgöz, O., M. Hagedorn, H. Holter, And Y. Wang (2018): "The Optimum Quantity of Capital and Debt," Working Paper, University of Oslo.

Acharya, S., E. Challe, and K. Dogra (2020): "Optimal Monetary Policy According to HANK," Federal Reserve Bank of New York Staff Reports.

Adjemian, S., H. Bastani, M. Juillard, F. Karamé, J. Maih, F. Mihoubi, G. Perendia, J. Pfeifer, M. Ratto, and S. Villemot (2011): "Dynare: Reference Manual, Version 4.6," Dynare Working Paper 1, CEPREMAP.

Ahn, S., G. Kaplan, B. Moll, T. Winberry, and C. Wolf (2017): "When Inequality Matters for Macro and Macro Matters for Inequality," in NBER Macroeconomics Annual 2017, volume 32, ed. by M. Eichenbaum and J. A. Parker, University of Chicago Press, $1-75$.

Aiyagari, S. R. (1994): "Uninsured Idiosyncratic Risk and Aggregate Saving," Quarterly Journal of Economics, 109, 659-684.

(1995): "Optimal Capital Income Taxation with Incomplete Markets, Borrowing Constraints, and Constant Discounting," Journal of Political Economy, 103, 1158-1175.

Aiyagari, S. R. And E. R. McGrattan (1998): "The Optimum Quantity of Debt," Journal of Monetary Economics, 42, 447-469.

Aruoba, B., J. Fernandez-Villaverde, and J. Rubio-Ramirez (2006): "Comparing Solution Methods for Dynamic Equilibrium Economies," Journal of Economic Dynamics and Control, 30, 2477-2508.

Auclert, A., B. Bardóczy, M. Rognlie, and L. Straub (2019): "Using the SequenceSpace Jacobian to Solve and Estimate Heterogeneous-Agent Models," Working Paper, Stanford University.

Bayer, C., R. Luetticke, L. Pham-Dao, and V. Tuaden (2019): "Precautionary Savings, Illiquid Assets, and the Aggregate Consequences of Shocks to Household Income Risk," Econometrica, 87, 255-290.

Bewley, T. F. (1983): "A Difficulty with the Optimum Quantity of Money," Econometrica, 51, 1485-1504.

Bhandari, A., D. Evans, M. Golosov, and T. J. Sargent (2020): "Inequality, Business Cycles, and Monetary-Fiscal Policy," Working Paper, New York University.

BilbiIe, F. (2008): "Limited Asset Markets Participation, Monetary Policy and (Inverted) Aggregate Demand Logic," Journal of Economic Theory, 140, 162-96. 
Bilbie, F. And X. Ragot (2020): "Optimal Monetary Policy and Liquidity with Heterogeneous Households," Review of Economic Dynamics, forthcoming.

Billingsley, P. P. (2012): Probability and Measure, Wiley-Blackwell, Anniversary Edition.

Boppart, T., P. Krusell, and K. Mitman (2018): "Exploiting Mit Shocks in Heterogeneous-Agent Economies: The Impulse Response as a Numerical Derivative," Journal of Economic Dynamics and Control, 89, 68-92.

Carrol, C. D. (2006): "The Method of Endogenous Grid Points for Solving Dynamic Stochastic Optimization Problems," Economic Letters, 91, 312-320.

Challe, E. (2020): "Uninsured Unemployment Risk and Optimal Monetary Policy in a Zero-Liquidity Economy," American Economic Journal: Macroeconomics, 12, 241-283.

Challe, E. And X. Ragot (2016): "Precautionary Saving over the Business Cycle," Economic Journal, 126, 135-164.

Chang, B., Y. Chang, And S.-B. Kim (2018): "Pareto Weights in Practice: Income Inequality and Tax Reform," Review of Economic Dynamics, 28, 181-204.

Chetty, R. (2009): "Sufficient Statistics for Welfare Analysis: A Bridge between Structural and Reduced-Form Methods," Annual Review of Economics, 1, 451-488.

Chetty, R. And A. Finkelstein (2013): "Social Insurance: Connecting Theory to Data," in Handbook of Public Economics, ed. by E. Saez, M. Feldstein, R. Chetty, and A. J. Auerbach, vol. 5, chap. 3, 111-193.

Chetty, R., A. Guren, D. Manoli, And A. Weber (2011): "Are Micro and Macro Labor Supply Elasticities Consistent? A Review of Evidence on the Intensive and Extensive Margins," American Economic Review, 101, 471-475.

Chien, Y., H. Cole, And H. Lustig (2011): "A Multiplier Approach to Understanding the Macro Implications of Household Finance," Review of Economic Studies, 78, 199-234.

(2012): "Is the Volatility of the Market Price of Risk Due to Intermittent Portfolio Rebalancing?" American Economic Review, 102, 2859-2896.

Cole, H. L. And F. Kubler (2012): "Recursive Contracts, Lotteries and Weakly Concave Pareto Sets," Review of Economic Dynamics, 15, 479-500.

Constantinides, G. M. And D. Duffie (1996): "Asset Pricing with Heterogeneous Consumers," Journal of Political Economy, 104, 219-240.

DÁvila, J., J. H. Hong, P. Krusell, And J.-V. Ríos-Rull (2012): "Constrained Efficiency in the Neoclassical Growth Model With Uninsurable Idiosyncratic Shocks," Econometrica, 80, 2431-2467.

Den HaAn, W. J. (2010): "Assessing the Accuracy of the Aggregate Law of Motion in Models with Heterogeneous Agents," Journal of Economic Dynamics and Control, 34, 79-99.

Den Haan, W. J. And A. Marcet (1994): "Accuracy in Simulations," Review of Economic Studies, 61, 3-17.

Dyrda, S. And M. Pedroni (2018): "Optimal Fiscal Policy in a Model with Uninsurable Idiosyncratic Shocks," Working Paper, University of Toronto. 
Faraglia, E., A. Marcet, O. Rigas, And A. Scott (2019): "Government Debt Management: The Long and the Short of It," Review of Economic Studies, 86, 2554-2604.

Green, E. (1994): "Individual-Level Randomness in a Nonatomic Population," Working Paper, University of Minnesota.

Heathсоте, J. (2005): "Fiscal Policy with Heterogeneous Agents and Incomplete Markets," Review of Economic Studies, 72, 161-188.

Heathcote, J., K. Storesletten, and G. L. Violante (2017): "Optimal Tax Progressivity: An Analytical Framework," Quarterly Journal of Economics, 132, 16931754 .

Hopenhayn, H. A. And E. C. Prescott (1992): "Stochastic Monotonicity and Stationary Distributions for Dynamic Economies," Econometrica, 60, 1387-1406.

Huggett, M. (1993): "The Risk Free Rate in Heterogeneous-Agent Incomplete-Insurance Economies," Journal of Economic Dynamics and Control, 17, 953-969.

ImrohoroĞLU, A. (1989): "Cost of Business Cycles with Indivisibilities and Liquidity Constraints," Journal of Political Economy, 97, 1364-1383.

Kolsrud, J., C. Landais, P. Nilsson, and J. Spinnewijn (2018): "The Optimal Timing of Unemployment Benefits: Theory and Evidence from Sweden," American Economic Review, 108, 985-1033.

Krueger, D. And A. Ludwig (2016): "On the Optimal Provision of Social Insurance: Progressive Taxation Versus Education Subsidies in General Equilibrium," Journal of Monetary Economics, 77, 72-98.

Krueger, D., K. Mittman, And F. Perri (2018): "On the Distribution of the Welfare Losses of Large Recessions," in Advances in Economics and Econometrics: Volume 2, Eleventh World Congress of the Econometric Society, ed. by B. Honoré, A. Pakes, M. Piazzesi, and L. Samuleson, Cambridge University Press, 143-184.

Krusell, P., T. Mukoyama, and A. Sahin (2010): "Labour-Market Matching with Precautionary Savings and Aggregate Fluctuations," Review of Economic Studies, 77, $1477-1507$.

Krusell, P., T. Mukoyama, and A. A. J. Smith (2011): "Asset Prices in a Huggett Economy," Journal of Economic Theory, 146, 812-844.

Krusell, P. And A. A. J. Smith (1998): "Income and Wealth Heterogeneity in the Macroeconomy," Journal of Political Economy, 106, 867-896.

Landais, C., P. Michaillat, And E. Saez (2018a): "A Macroeconomic Approach to Optimal Unemployment Insurance: Applications," American Economic Journal: Economic Policy, 10, 182-216.

(2018b): "A Macroeconomic Approach to Optimal Unemployment Insurance: Theory," American Economic Journal: Economic Policy, 10, 152-181.

LeGrand, F., A. Martin-Baillon, and X. Ragot (2021): "Should Monetary Policy Care About Redistribution? Optimal Fiscal and Monetary Policy with Heterogeneous Agents," Working Paper, SciencesPo.

Lucas, R. E. J. (1975): "An Equilibrium Model of the Business Cycle," Journal of Political Economy, 83, 1113-1144. 
(1990): "Liquidity and Interest Rates," Journal of Economic Theory, 50, 237-254.

Luenberger, D. G. And Y. Ye (2016): Linear and Nonlinear Programming, Springer, 3rd ed.

Marcet, A. And R. Marimon (2019): "Recursive Contracts," Econometrica, 87, 15891631.

McKay, A. And R. Reis (2020): "Optimal Automatic Stabilizers," Review of Economic Studies, Forthcoming.

Mitman, K. and S. Rabinovich (2015): "Optimal Unemployment Insurance in an Equilibrium Business-Cycle Model," Journal of Monetary Economics, 71, 99-118.

NuÑO, G. AND B. Moll (2018): "Social Optima in Economies with Heterogeneous Agents," Review of Economic Dynamics, 48, 150-180.

Pavoni, N., C. Sleet, And M. Messner (2018): "The Dual Approach to Recursive Optimization: Theory and Examples," Econometrica, 86, 133-172.

QUADRINI, V. (1999): "The Importance of Entrepreneurship for Wealth Concentration and Mobility," Review of Income and Wealth, 45, 1-19.

Ravn, M. O. And V. Sterk (2017): "Job Uncertainty and Deep Recessions," Journal of Monetary Economics, 90, 125-141.

Reiter, M. (2009): "Solving Heterogeneous-Agent Models by Projection and Perturbation," Journal of Economic Dynamics and Control, 33, 649-665.

Rios-Rull, V. (1999): "Computation of Equilibria in Heterogenous Agent Models: An Introduction," Oxford University Press.

Rouwenhorst, G. K. (1995): "Asset Pricing Implications of Equilibrium Business Cycle Models," in Structural Models of Wage and Employment Dynamics, ed. by T. Cooley, Princeton: Princeton University Press, 201-213.

Shimer, R. (2003): "The Cyclical Behavior of Equilibrium Unemployment and Vacancies," American Economic Review, 95, 25-49.

Stokey, N. L., R. E. J. Lucas, And E. C. Prescott (1989): Recursive Methods in Economic Dynamics, Harvard University Press.

Werning, I. (2015): "Incomplete Markets and Aggregate Demand," NBER Working Paper 21448, National Bureau of Economic Research.

Winberry, T. (2018): "A Method for Solving and Estimating Heterogeneous Agent Macro Models," Quantitative Economics, 9, 1123-1151.

Young, E. (2010): "Solving the Incomplete Markets Model with Aggregate Uncertainty using the Krusell-Smith Algorithm and Non-Stochastic Simulations," Journal of Economic Dynamics and Control, 34, 36-41. 


\section{Appendix}

This Appendix is split into five parts. Section A presents a micro-foundation theory of the truncation method. Section B contains the proofs of the paper. Section C presents the details of the truncation method. Section D contains a number of robustness checks and additional results about the implementation of our numerical implementation. Finally, Section E presents supplemental theoretical results.

\section{A Decentralizing the truncation method}

In this section, we explain how the truncation method of Section 4 can be seen as the outcome of a decentralized mechanism. We show that the truncated allocation is the market outcome of an island economy (Section A.1) - see Lucas, 1975, 1990, or Heathcote et al., 2017 for a more recent reference. We also prove that the island economy can be decentralized by a well-chosen fiscal system of lump-sum transfers (A.2). We denote the truncation length by $N \geq 0$.

\section{A.1 The island metaphor}

Island description. There are $S^{N}$ different islands, where $S$ is the cardinal of $\mathcal{S}$. Agents with the same idiosyncratic history for the last $N$ periods are located on the same island. Any island is represented by a vector $s^{N}=\left(s_{-N+1}^{N}, \ldots, s_{0}^{N}\right) \in \mathcal{S}^{N}$ summarizing the last $N$-period idiosyncratic history of all island inhabitants. At the beginning of each period, agents face a new idiosyncratic shock. Agents with history $\hat{s}^{N}$ in the previous period are endowed with the new history $s^{N}$ in the current period, and have to move to the corresponding island. The history $s^{N}$ will be said to be a continuation of $\hat{s}^{N}$, and will be denoted by $s^{N} \succeq \hat{s}^{N}$. The probability of transitioning from island $\hat{s}^{N}$ at $t-1$ to island $s^{N}$ at date $t$ is denoted by $\Pi_{t, \hat{s}^{N}, s^{N}}$, defined in $(21)$. The island sizes $\left(S_{t, s^{N}}\right)_{s^{N} \in \mathcal{S}^{N}}$ can be deduced from these probabilities and are defined in recursion (22).

The specification $N=0$ (one island) corresponds to the standard representative-agent model. Symmetrically, the case $N=\infty$ corresponds to a standard incomplete-market economy with aggregate shocks, as in Krusell and Smith (1998). 
The quasi-planner. The quasi-planner maximizes the welfare of agents, attributing an identical weight to all agents and behaving as a price-taker. ${ }^{22}$ The quasi-planner can freely transfer resources among agents on the same island, but cannot do so across islands. All agents belonging to the same island are treated identically and therefore receive the same allocation, as is consistent with welfare maximization. For island $s^{N}$, the quasi-planner will choose the per capita consumption level $c_{t, s^{N}}$, the labor supply $l_{t, s^{N}}$, and the savings $a_{t, s^{N}}$.

Wealth pooling and heterogeneity reduction. At the beginning of each period $t$, agents learn about their current idiosyncratic shock and move from island $\hat{s}^{N}$ to island $s^{N}$. Agents take their wealth - equal to $a_{t-1, \hat{s}^{N}}-$ with them when they move. On island $s^{N}$, the wealth of all agents coming from island $\hat{s}^{N}$ (equal to $S_{t-1, \hat{s}^{N}} \Pi_{t, \hat{s}^{N}, s^{N}} a_{t-1, \hat{s}^{N}}$ ) - and for all islands $\hat{s}^{N}$ - is pooled together and then equally divided among the $S_{t, s^{N}}$ agents of island $s^{N}$. Therefore, at the beginning of period $t$, each agent of $s^{N}$ holds wealth $\tilde{a}_{t, s^{N}}$, defined in equation (24). We denote by $\left(a_{-1, s^{N}}\right)_{s^{N} \in \mathcal{S}^{N}}$ the initial wealth endowment.

Agents face island-specific preference shifters, denoted by $\xi_{s^{N}}$, which multiply their utility function. The quasi-planner's program can be expressed as:

$$
\begin{gathered}
\max _{\left(c_{t, s^{N}}, l_{t, s^{N}}, a_{t, s^{N}}, \tilde{a}_{t, s^{N}}\right)_{t \geq 0, s^{N} \in \mathcal{S}^{N}}} \mathbb{E}_{0} \sum_{t=0}^{\infty} \beta^{t} \sum_{s^{N} \in \mathcal{S}^{N}} S_{t, s^{N}} \xi_{s^{N}} U\left(c_{t, s^{N}}, \hat{l}_{t, s^{N}}\right), \\
a_{t, s^{N}}+c_{t, s^{N}}=\left(\left(1-\tau_{t}\right) l_{t, s^{N}} 1_{e_{0}^{N}=e}+\phi_{t} l_{t, s^{N}, e^{N}} 1_{e_{0}^{N}=u}\right) y_{0}^{N} w_{t}+\left(1+r_{t}\right) \tilde{a}_{t, s^{N}}\left(s^{N} \in \mathcal{S}^{N}\right), \\
c_{t, s^{N}}, l_{t, s^{N}} \geq 0, a_{t, s^{N}} \geq-\bar{a}\left(s^{N} \in \mathcal{S}^{N}\right)
\end{gathered}
$$

and subject to the law of motion for $\left(S_{t, s^{N}}\right)_{t \geq 0}^{s^{N} \in \mathcal{S}^{N}}$, and to the definition of $\left(\tilde{a}_{t, s^{N}}\right)_{t \geq 0}^{s^{N} \in \mathcal{S}^{N}}$. Note that initial island sizes $\left(S_{-1, s^{N}}\right)_{s^{N} \in \mathcal{S}^{N}}$ and initial wealth $\left(a_{-1, s^{N}}\right)_{s^{N} \in \mathcal{S}^{N}}$ are given. ${ }^{23}$

As the objective function is increasing and concave, constraints are linear (i.e., the admissible set is convex), and the existence of the equilibrium can be proved using standard techniques (see Stokey et al., 1989, Chap. 15 and 16). We therefore omit this proof in the interest of conciseness.

\footnotetext{
${ }^{22}$ As the quasi-planner does not internalize the effect of its choices on prices, the allocation is not constrained-efficient, and the distortions identified by Dávila et al. (2012) are present in the equilibrium allocation.

${ }^{23}$ In equation (37), $l_{t, s^{N}, e}$ is the labor supply of an employed agent with productivity $y_{0}^{N}$, which determines the UI benefits of unemployed agents of history $s^{N}$. Furthermore, as in (10), $\hat{l}_{t, s^{N}}=l_{t, s^{N}} 1_{e_{0}^{N}=e}+\zeta_{y_{0}^{N}} 1_{e_{0}^{N}=u}$.
} 
The first-order conditions of the quasi-planner's program are the same as those derived in the main text for the truncated economy (same notation):

(39) $\xi_{s^{N}} U_{c}\left(c_{t, s^{N}}, \hat{l}_{t, s^{N}}\right)=\beta \mathbb{E}_{t}\left[\sum_{\tilde{s}^{N} \succeq s^{N}} \Pi_{t+1, s^{N}, \tilde{s}^{N}} \xi_{\tilde{s}^{N}} U_{c}\left(c_{t+1, \tilde{s}^{N}}, \hat{l}_{t+1, \tilde{s}^{N}}\right)\left(1+r_{t+1}\right)\right]+\nu_{t, s^{N}}$,

(41) $\nu_{t, s^{N}}\left(a_{t, s^{N}}+\bar{a}\right)=0$ and $\nu_{t, s^{N}} \geq 0$.

Market clearing and equilibrium. The clearing for labor and capital markets implies equations (29). We can now state our sequential equilibrium definition, which is similar to the definition of the truncated equilibrium in the main text (Definition 2).

Definition 3 (Sequential equilibrium) A sequential truncated competitive equilibrium is a collection of individual allocations $\left(c_{t, s^{N}}, l_{t, s^{N}}, a_{t, s^{N}}, \tilde{a}_{t, s^{N}}\right)_{t \geq 0}^{s^{N} \in \mathcal{S}^{N}}$, of island population sizes $\left(S_{t, s^{N}}\right)_{t \geq 0}^{s^{N} \in \mathcal{S}^{N}}$, of aggregate quantities $\left(L_{t}, K_{t}, Y_{t}\right)_{t \geq 0}$, of price processes $\left(w_{t}, r_{t}\right)_{t \geq 0}$, and of UI policy $\left(\tau_{t}, \phi_{t}\right)_{t \geq 0}$, such that, for an initial distribution of island population and wealth $\left(S_{-1, s^{N}}, a_{-1, s^{N}}\right)_{s^{N} \in \mathcal{S}^{N}}$, and for initial values of capital stock $K_{-1}$ and of the initial aggregate shock $z_{-1}$, we have:

1. given prices, individual strategies $\left(c_{t, s^{N}}, l_{t, s^{N}}, a_{t, s^{N}}, \tilde{a}_{t, s^{N}}\right)_{t \geq 0}^{s^{N} \in \mathcal{S}^{N}}$ solve the agents' optimization program in equations (36)-(38);

2. island sizes and beginning-of-period individual wealth $\left(S_{t, s^{N}}, \tilde{a}_{t, s^{N}}\right)_{t \geq 0}^{s^{N} \in \mathcal{S}^{N}}$ are consistent with the laws of motion (22) and (24);

3. capital and labor markets clear at all dates: for any $t \geq 0$, equations (29) hold;

4. the UI budget is balanced at all dates: equation (14) holds for all $t \geq 0$;

5. factor prices $\left(w_{t}, r_{t}\right)_{t \geq 0}$ are consistent with the firm's program (3).

The sequential equilibrium has a simple structure defined at each date by $5 S^{N}+5$ variables and $5 S^{N}+5$ equations for a given UI policy $\left(\tau_{t}, \phi_{t}\right)_{t \geq 0}$. The equilibrium features a finite number of different allocations, characterized by the $N$-period history of agents.

\section{A.2 A decentralization mechanism}

We now prove that the finite-state equilibrium of Definition 3 can be decentralized through fiscal transfers, which are shown to measure the degree of idiosyncratic risk sharing achieved 
by asset pooling in the island economy. The economy is now similar to that in Section $2-$ in particular, agents are expected-utility maximizers - except for two differences. First, agents are endowed with so-called preference shifters. Second, at each date, each agent receives a lump-sum transfer $\Gamma_{N+1}$, which is contingent on her individual history $s^{N+1}$ over the previous $N+1$ periods. This fiscal system will be key in mimicking the pooling operation of Section A.1. Using standard recursive notation, the agents' program can be written as: ${ }^{24}$

$$
\begin{gathered}
V\left(a, s^{N+1}, X\right)=\max _{a^{\prime}, c, l} \xi_{s^{N}} U(c, \hat{l})+\beta \mathbb{E}\left[\sum_{\left(s^{N+1}\right)^{\prime}} \Pi_{s^{N+1},\left(s^{N+1}\right)^{\prime}}^{\prime} V\left(a^{\prime},\left(s^{N+1}\right)^{\prime}, X^{\prime}\right)\right] \\
a^{\prime}+c-(1+r(X)) a=\left((1-\tau) l 1_{e_{0}^{N}=e}+\phi l_{y, e} 1_{e_{0}^{N}=u}\right) w(X)+\Gamma_{N+1}\left(s^{N+1}, X\right) \\
\hat{l}=l 1_{e_{0}^{N}=e}+\zeta_{y_{0}^{N}} 1_{e_{0}^{N}=u} \\
c, l \geq 0, a^{\prime} \geq-\bar{a}
\end{gathered}
$$

where $l_{y, e}$ denotes the labor supply of an employed agent with productivity $y$, and where the state vector $X$ encompasses all variables necessary to forecast prices, including aggregate shocks. Compared to the economies studied by Huggett (1993) and Aiyagari (1994), the individual history $s^{N+1}$ is a state variable, as it determines the transfer $\Gamma_{N+1}\left(s^{N+1}, X\right)$.

We now state our result, which explains that we can find a particular set of transfers denoted by $\left(\Gamma_{N+1}^{*}\left(s^{N+1}, X\right)\right)_{s^{N+1} \in \mathcal{S}^{N+1}}$ - such that the decentralized economy allocations match those of the island economy.

Proposition 4 (Finite state space) A set of balanced transfers exists, that are denoted by $\left(\Gamma_{N+1}^{*}\left(s^{N+1}, X\right)\right)_{s^{N+1} \in \mathcal{S}^{N+1}}$, such that any optimal allocation of the island program (36)-(38) is also a solution to the decentralized program (42)-(45).

Proposition 4, proved below, states that the island program presented in Section A.1 can be decentralized by the balanced lump-sum transfers $\left(\Gamma_{N+1}^{*}\left(s^{N+1}, X\right)\right)_{s^{N+1} \in \mathcal{S}^{N+1}}$ (shortened to $\Gamma_{N+1}^{*}$ henceforth). This transfer is formally provided in equation (47).

The transfers $\Gamma_{N+1}^{*}$ mimic the wealth pooling of the island economy (equation (24)), when agents transfer from one island to another. It consists of two steps: (i) putting together the beginning-of-period wealth of all agents with the same idiosyncratic history

\footnotetext{
${ }^{24}$ As standard, we denote the current savings choice by $a^{\prime} ; a$ is thus the beginning-of-period wealth.
} 
for the last $N$ periods, independently of their idiosyncratic status $N+1$ periods ago, and (ii) redistributing consistently the same amount to agents with the same idiosyncratic history for $N$ periods, such that there are only $S^{N}$ possible wealth levels. The transfers $\left(\Gamma_{N+1}^{*}\right)$ operate a strict redistribution among agents and thus sum up to zero.

\section{Proof of Proposition 4}

We use a guess-and-verify strategy. The transfer is constructed such that all agents with the same $N$-period history have the same after-transfer wealth. The measure of agents with history $s^{N}$ follows the same law of motion as (22) in the main text and is equal to $S_{s^{N}}$. If agents with the same history $\left(\hat{s}^{N}, s\right), s \in \mathcal{S}$ have the same beginning-of-period wealth $a_{\hat{s}^{N}}$, the after-transfer wealth, denoted by $\hat{a}_{s^{N}}$, of agents with history $s^{N} \succeq \hat{s}^{N}$ is:

$$
\hat{a}_{s^{N}}^{\prime}=\sum_{\tilde{s}^{N} \in \mathcal{S}^{N}} \frac{S_{\tilde{s}^{N}}}{S_{s^{N}}} \Pi_{\tilde{s}^{N}, s^{N}} a_{\tilde{s}^{N}}^{\prime}
$$

such that agents with the same history hold the same wealth. By construction, $\hat{a}_{s^{N}}$ follows dynamics similar to the "after-pooling" wealth $\tilde{a}_{t, s^{N}}$ in the island economy of equation (24). The transfer scheme denoted by $\left(\Gamma_{N+1}^{*}\left(s^{N+1}, X\right){ }_{s^{N+1} \in \mathcal{S}^{N+1}}\right.$ that enables all agents with the same history to have the same wealth is:

$$
\Gamma_{N+1}^{*}\left(s^{N+1}, X\right)=(1+r)\left(\hat{a}_{s^{N}}-a_{\hat{s}^{N}}\right),
$$

where we use $s^{N+1}=\left(\hat{s}^{N}, s\right)=\left(s_{N}, s^{N}\right)$ (in the former notation, $s^{N+1}$ is seen as the history $\hat{s}^{N} \in \mathcal{S}^{N}$ with the successor state $s \in \mathcal{S}$, while in the latter notation, $s^{N+1}$ is seen as the state $s_{N} \in \mathcal{S}$ followed by history $s^{N} \in \mathcal{S}^{N}$ ). The transfer $\Gamma_{N+1}^{*}$ defined in (47) replaces the beginning-of-period wealth $(1+r) a_{\hat{s}^{N}}$ with the average wealth $(1+r) \hat{a}_{s^{N}}$, which only depends on the last $N$-period history. Since there is a continuum with mass $S_{\tilde{s}^{N}}$ of agents with history $\tilde{s}^{N}$, in which each agent is atomistic, all agents take the transfer $\Gamma_{N+1}^{*}$ as given.

Finally, it is easy to check that the transfer scheme is balanced in each period. Using the definition (46) of $\hat{a}_{s^{N}}$, we obtain for $s^{N}=\left(s_{N-1}^{N}, \ldots, s_{1}^{N}, s_{0}^{N}\right) \in \mathcal{S}^{N}, S_{s^{N}} \hat{a}_{s^{N}}=$ $\sum_{\hat{s}^{N} \in \mathcal{S}^{N}} S_{\hat{s}^{N}} \prod_{\hat{s}^{N}, s^{N}} a_{\hat{s}^{N}}=\sum_{\hat{s} \in \mathcal{S}} S_{\left(\hat{s}, s_{N-1}^{N}, \ldots, s_{1}^{N}\right)} M_{s_{1}^{N}, s_{0}^{N}} a_{\left(\hat{s}, s_{N-1}^{N}, \ldots, s_{1}^{N}\right)}$. Therefore, we deduce that: $\sum_{\tilde{s} \in \mathcal{S}} S_{\left(\tilde{s}, s^{N}\right)} \Gamma_{N+1}^{*}\left(\tilde{s}, s^{N}\right)=(1+r)\left[\sum_{\tilde{s} \in \mathcal{S}} S_{\left(\tilde{s}, s^{N}\right)}\left(\hat{a}_{s^{N}}-a_{\left(\tilde{s}, s_{N-1}^{N}, \ldots, s_{1}^{N}\right)}\right)\right]=0$, where 
the last equality comes from the definition of $\hat{a}_{s^{N}}$ in equation (46).

\section{B Proofs}

\section{B.1 The original Ramsey program}

FOC with respect to saving choices $a_{t}^{i}$. Using equations (11), we can show for aggregate quantities that $\frac{\partial K_{t-1}}{\partial a_{t}^{i}}=\frac{\partial L_{t}}{\partial a_{t}^{i}}=0$, and:

$$
\frac{\partial K_{t}}{\partial a_{t}^{i}}=1, \frac{\partial L_{t+1}}{\partial a_{t}^{i}}=\frac{\varphi L_{t+1} \frac{F_{K L, t+1}}{F_{L, t+1}}}{1-\varphi L_{t+1} \frac{F_{L L, t+1}}{F_{L, t+1}}}, \frac{\partial w_{t+1}}{\partial a_{t}^{i}}=\frac{F_{K L, t+1}}{1-\varphi L_{t+1} \frac{F_{L L, t+1}}{F_{L, t+1}}} .
$$

After some manipulations, and using $F(K, L)=K^{\alpha} L^{1-\alpha}-\delta K$, we obtain for $c_{t}^{i}$ and $l_{t}^{i}$ :

$$
\begin{aligned}
\frac{\partial l_{t}^{i}}{\partial a_{t}^{j}} & =0, \frac{\partial l_{t+1}^{i}}{\partial a_{t}^{j}}=\varphi \frac{\alpha K_{t}^{-1}}{1+\alpha \varphi} l_{t+1}^{i} 1_{e_{t}^{i}=e}, \frac{\partial c_{t}^{i}}{\partial a_{t}^{j}}=-1_{j=i}, \\
\frac{\partial c_{t+1}^{i}}{\partial a_{t}^{j}} & =\left(1+r_{t+1}\right) 1_{i=j}+\frac{F_{K K, t+1}}{1+\alpha \varphi} a_{t-1}^{i} \\
\quad+ & \left(\left(1-\tau_{t+1}\right) 1_{e_{t+1}^{i}=e}+\frac{S_{t+1, e}}{S_{t+1, u}} \tau_{t+1} 1_{e_{t+1}^{i}=u}\right) l_{t+1, e}^{i} y_{t+1}^{i}(1+\varphi) \frac{\alpha w_{t} K_{t}^{-1}}{1+\alpha \varphi} .
\end{aligned}
$$

The derivative of the Lagrangian with respect to $a_{t}^{j}$ implies, using (17):

$$
\begin{aligned}
& \psi_{t}^{j}=\beta \mathbb{E}_{t}\left[\int_{i} \psi_{t+1}^{i}\left(\frac{\partial c_{t+1}^{i}}{\partial a_{t}^{i}}-\chi^{-1} l_{t+1}^{i, \frac{1}{\varphi}} \frac{\partial l_{t+1}^{i}}{\partial a_{t}^{i}}\right) \ell(d i)\right] \\
& +\beta \mathbb{E}_{t}\left[\int_{i}\left(F_{K K, t+1}+F_{K L, t+1} \frac{\varphi L_{t+1} \frac{F_{K L, t+1}}{F_{L, t+1}}}{1-\varphi L_{t+1} \frac{F_{L L, t+1}}{F_{L, t+1}}}\right) \lambda_{t}^{i} U_{c, t+1}^{i} \ell(d i)\right] .
\end{aligned}
$$

With $\frac{F_{K L}^{2}-F_{K K} F_{L L}}{F_{L}}=0$ and the expression of $\frac{\partial c_{t+1}^{i}}{\partial a_{t}^{i}}-\chi^{-1} l_{t+1}^{i, \frac{1}{\varphi}} \frac{\partial l_{t+1}^{i}}{\partial a_{t}^{i}}$, we obtain FOC (18).

FOC with respect to replacement rate $\phi_{t}$. Rather than computing the derivative with respect to $\phi_{t}$, we do so with respect to $\tau_{t}$. For aggregate quantities, we obtain quite directly: $\frac{\partial L_{t}}{\partial \tau_{t}}=-\frac{\varphi}{1+\alpha \varphi} \frac{L_{t}}{1-\tau_{t}}, \frac{\partial K_{t-1}}{\partial \tau_{t}}=0$. The computation for individual choices is lengthier, and yields:

$$
\begin{aligned}
\frac{\partial l_{t}^{i}}{\partial \tau_{t}} & =-\frac{\varphi}{1-\tau_{t}} l_{t}^{i} 1_{e_{t}^{i}=e}, \\
\frac{\partial c_{t}^{i}}{\partial \tau_{t}} & =-\frac{\alpha \varphi \frac{w_{t}}{1-\tau_{t}} \frac{L_{t}}{K_{t-1}}}{1+\alpha \varphi} a_{t-1}^{i}+\frac{S_{t, e}}{S_{t, u}} \frac{l_{t}^{i}}{1-\tau_{t}} y_{t}^{i} w_{t} 1_{e_{t}^{i}=u}\left(1-\tau_{t} \frac{1+\varphi}{1+\alpha \varphi}\right)-1_{e_{t}^{i}=e} l_{t}^{i} y_{t}^{i} w_{t} \frac{1+\varphi}{1+\alpha \varphi} .
\end{aligned}
$$


Using the Lagrangian expression, we obtain after substituting for $\psi_{t}^{i}$ and partial derivatives:

$$
\begin{aligned}
0 & =\int_{i} \psi_{t}^{i}\left(\frac{S_{t, e}}{S_{t, u}} \frac{l_{t}^{i}}{1-\tau_{t}} y_{t}^{i} w_{t} \frac{1-\tau_{t}-\varphi(1-\alpha)}{1+\alpha \varphi} 1_{e_{t}^{i}=u}-1_{e_{t}^{i}=e} l_{t}^{i} y_{t}^{i} w_{t} \frac{1}{1+\alpha \varphi}\right) \ell(d i) \\
& -\frac{\alpha \varphi}{1+\alpha \varphi} \frac{w_{t}}{1-\tau_{t}} \frac{L_{t}}{K_{t-1}} \int_{i}\left(\lambda_{t-1}^{i} U_{c, t}^{i}+\psi_{t}^{i} a_{t-1}^{i}\right) \ell(d i)
\end{aligned}
$$

which yields equation (19) after some rearrangements.

\section{B.2 The Ramsey program in the truncated economy}

The computations are similar to those in the original Ramsey program (Section B.1).

\section{B.2.1 Rewriting the Ramsey program}

In the remainder, we use the notation $U_{c, t, s^{N}}=u^{\prime}\left(c_{t, s^{N}}-\chi^{-1} \frac{\hat{l}_{t, s^{N}}^{1+1 / \varphi}}{1+1 / \varphi}\right)$, and similarly for $U_{c c, t, s^{N}}, U_{c l, t, s^{N}}$, and $U_{l l, t, s^{N}}$. The planner's program can be written as:

$$
\begin{gathered}
\max _{\left(\left(a_{t, s^{N}}, c_{t, s^{N}}, l_{t, s^{N}}\right)_{s^{N}}, \phi_{t}, \tau_{t}, r_{t}, w_{t}\right)} \mathbb{E}_{t \geq 0}\left[\sum_{t=0}^{\infty} \beta^{t} \sum_{s^{N} \in \mathcal{S}^{N}} S_{t, s^{N}} \xi_{t, s^{N}} U\left(c_{t, s^{N}}, \hat{l}_{t, s^{N}}\right)\right] \\
\xi_{t, s^{N}} U_{c, t, s^{N}}-\nu_{t, s^{N}}=\beta \mathbb{E}_{t}\left[\left(1+r_{t+1}\right) \sum_{\tilde{s}^{N} \in \mathcal{S}^{N}} \Pi_{t+1, s^{N} \tilde{s}^{N}} \xi_{t+1, \tilde{s}^{N}} U_{c, t+1, s^{N}}\right]
\end{gathered}
$$

and subject to (25), (29), (14), and (3). Let $\beta^{t} S_{t, s^{N}} \lambda_{t, s^{N}}$ be the Lagrange multiplier on the Euler equation. With $\nu_{t, s^{N}} \lambda_{t, s^{N}}=0$, the planner's objective, denoted by $J$, becomes:

(51) $J=\mathbb{E}_{0} \sum_{t, s^{N}} \beta^{t}\left(S_{t, s^{N}} \xi_{t, s^{N}} U\left(c_{t, s^{N}}, \hat{l}_{t, s^{N}}\right)-\left(\lambda_{t, s^{N}}-\left(1+r_{t}\right) \tilde{\lambda}_{t, s^{N}}\right) \xi_{t, s^{N}} U_{c}\left(c_{t, s^{N}}, \hat{l}_{t, s^{N}}\right)\right)$.

The Ramsey program consists in maximizing $J$ in $(51)$ over $\left(\left(a_{t, s^{N}}, c_{t, s^{N}}, l_{t, s^{N}}\right)_{s^{N}}, \phi_{t}, \tau_{t}\right)_{t \geq 0}$ subject to the relevant constraints. As in the original case, the Lagrangian can be seen as depending only on saving choices $\left(a_{t, s^{N}}\right)$ and the replacement rate $\phi_{t}$.

FOC with respect to saving choices $a_{t, s^{N}} \quad$ Using equations (29), we can show for aggregate quantities that:

$$
\frac{\partial K_{t}}{\partial a_{t, s^{N}}}=S_{t, s^{N}}, \frac{\partial K_{t-1}}{\partial a_{t, s^{N}}}=0, \frac{\partial L_{t+1}}{\partial a_{t, s^{N}}}=\frac{\varphi L_{t+1} \frac{F_{K L, t+1}}{F_{L, t+1}}}{1-\varphi L_{t+1} \frac{F_{L L, t+1}}{F_{L, t+1}}} S_{t, s^{N}}, \frac{\partial L_{t}}{\partial a_{t, s^{N}}}=0 .
$$


After some manipulations, we obtain for individual choices (labor and consumption):

$$
\begin{gathered}
\frac{\partial l_{t, \tilde{s}^{N}}}{\partial a_{t, s^{N}}}=0, \frac{\partial l_{t+1, \tilde{s}^{N}}}{\partial a_{t, s^{N}}}=\varphi \frac{\frac{F_{K L, t+1}}{F_{L, t+1}}}{1-\varphi L_{t+1} \frac{F_{L L, t+1}}{F_{L, t+1}}} S_{t, s^{N}} l_{\tilde{s}^{N}, e, t+1} 1_{e_{\tilde{s}^{N}}=e}, \frac{\partial c_{t, \tilde{s}^{N}}}{\partial a_{t, s^{N}}}=-1_{s^{N}=\tilde{s}^{N}}, \\
\frac{\partial c_{t+1, \tilde{s}^{N}}}{\partial a_{t, s^{N}}}=\left(1+F_{K, t+1}\right) \Pi_{t+1, s^{N} \tilde{s}^{N}}+\frac{F_{K K, t+1}+\varphi L_{t+1} \frac{F_{K L, t+1}^{2}-F_{K K, t+1} F_{L L, t+1}}{F_{L, t+1}}}{1-\varphi L_{t+1} \frac{F_{L L, t+1}}{F_{L, t+1}}} S_{t, s^{N}} \tilde{a}_{t, s^{N}} \\
+\left(\left(1-\tau_{t+1}\right) 1_{\tilde{s}_{\tilde{s}^{N}}=e}+\frac{S_{t+1, e}}{S_{t+1, u}} \tau_{t+1} 1_{e_{\tilde{s}^{N}}=u}\right) l_{\tilde{s}^{N}, e, t+1} \tilde{y}(1+\varphi) \frac{F_{K L, t+1}}{1-\varphi L_{t+1} \frac{F_{L L, t+1}}{F_{L, t+1}}} S_{t, s^{N}} .
\end{gathered}
$$

We deduce for $\frac{\partial C_{t+1, \tilde{s}^{N}}}{\partial a_{t, s^{N}}}=\frac{\partial c_{t+1, \tilde{s}^{N}}}{\partial a_{t, s^{N}}}-\chi^{-1} l_{t+1, \tilde{s}^{N}}^{\frac{1}{\varphi}} \frac{\partial l_{t+1, \tilde{s}^{N}}}{\partial a_{t, s^{N}}}$ :

$$
\begin{aligned}
& \frac{\partial C_{t+1, \tilde{s}^{N}}}{\partial a_{t, s^{N}}}=\left(1+r_{t+1}\right) \Pi_{t+1, s^{N} \tilde{s}^{N}}+\frac{F_{K K, t+1}+\varphi L_{t+1} \frac{F_{K L, t+1}^{2}-F_{K K, t+1} F_{L L, t+1}}{F_{L, t+1}}}{1-\varphi L_{t+1} \frac{F_{L L, t+1}}{F_{L, t+1}}} S_{t, s^{N}} \tilde{a}_{t, s^{N}} \\
& \quad+\left(\left(1-\tau_{t+1}\right) 1_{\tilde{s}^{N}=e}+\frac{S_{t+1, e}}{S_{t+1, u}} \tau_{t+1} 1_{\tilde{s}^{N}=u}\right) l_{\tilde{s}^{N}, e, t+1} \tilde{y}(1+\varphi) \frac{F_{K L, t+1}}{1-\varphi L_{t+1} \frac{F_{L L, t+1}}{F_{L, t+1}}} S_{t, s^{N}} \\
& \quad-\left(1-\tau_{t+1}\right) y_{\tilde{s}^{N}} \varphi \frac{F_{K L, t+1}}{1-\varphi L_{t+1} \frac{F_{L L, t+1}}{F_{L, t+1}}} S_{t, s^{N}} l_{\tilde{s}^{N}, e, t+1}
\end{aligned}
$$

Note that using $F(K, L)=K^{\alpha} L^{1-\alpha}-\delta K$, we have: $\frac{F_{K L}^{2}-F_{K K} F_{L L}}{F_{L}}=0 ; \quad L \frac{F_{L L}}{F_{L}}=$ $-\alpha ; F_{K L}=F_{L} \frac{\alpha}{K_{t}}$ and $F_{K K}=-\alpha F_{L} \frac{L}{K^{2}}$. Simplifying the partial derivatives of $F$ and using (52), the derivative of the Lagrangian with respect to $a_{t, s^{N}}$ implies equation (33).

FOC with respect to $\phi_{t}$ Rather than computing the derivative with respect to $\phi_{t}$, we do so with respect to $\tau_{t}$. For aggregate quantities, we obtain quite directly: $\frac{\partial L_{t}}{\partial \tau_{t}}=-\frac{\varphi \frac{L_{t}}{1-\tau_{t}}}{1-\varphi L_{t} \frac{F_{L L, t}}{F_{L, t}}}$, $\frac{\partial K_{t-1}}{\partial \tau_{t}}=0$. The computation for individual choices is lengthier, and yields:

$$
\begin{aligned}
& \frac{\partial l_{t, \tilde{s}^{N}}}{\partial \tau_{t}}=-\frac{\frac{\varphi}{1-\tau_{t}} l_{t, \tilde{s}^{N}}}{1-\varphi L_{t} \frac{F_{L L, t}}{F_{L, t}}} 1_{\tilde{\tilde{s}}^{N}=e}, \\
& \frac{\partial c_{t, \tilde{s}^{N}}}{\partial \tau_{t}}=-\frac{\varphi \frac{L_{t}}{1-\tau_{t}}}{1-\varphi L_{t} \frac{F_{L L, t}}{F_{L, t}}} F_{K L, t} \tilde{a}_{t, \tilde{s}^{N}}+\left(1-\tau_{t}\right)^{\varphi-1} \frac{S_{t, e}}{S_{t, u}} \chi^{\varphi} \tilde{y}^{\varphi+1} F_{L, t}^{1+\varphi} 1_{e_{\tilde{s}^{N}}=u} \\
&-\left(\left(1-\tau_{t}\right) 1_{e_{\tilde{s}} N}=e\right. \\
&
\end{aligned}
$$

Using the partial derivatives of $F$ and previous computations, we obtain equation (34). 


\section{B.3 Proof of Proposition 2}

Although the proof is technical, the idea can be summarized quite simply. The sequence of aggregated allocations (23) as a function of truncation length can be seen as a sequence of conditional expectations with a well-chosen filtration. The martingale convergence theorem then enables us to conclude.

\section{B.3.1 Proof of the first part of Proposition 2}

We consider a truncation length $N>0$, and a steady-state allocation, denoted by $\left(a_{s^{\infty}}, c_{s^{\infty}}, l_{s^{\infty}}, \nu_{s^{\infty}}\right)_{s^{\infty} \in \mathcal{S}^{\infty}}$, of an original Bewley model given a UI policy $(\phi, \tau)$. We construct the aggregated allocation $\left(c_{s^{N}}, l_{s^{N}}, a_{s^{N}}, \nu_{s^{N}}\right)_{s^{N} \in \mathcal{S}^{N}}$ using equation (23) at the steady state and we aim to show that when $N \rightarrow \infty$, we have $\left(c_{s^{N}}, l_{s^{N}}, a_{s^{N}}, \nu_{s^{N}}\right)_{s^{N} \in \mathcal{S}^{N}} \longrightarrow_{N}$ $\left(a_{s^{\infty}}, c_{s^{\infty}}, l_{s^{\infty}}, \nu_{s^{\infty}}\right)_{s^{\infty} \in \mathcal{S}^{\infty}}$, almost surely. The proof is split in several steps: (i) we introduce some formal notation and construct the probability space related to the set of infinite histories $\mathcal{S}^{\infty}$; (ii) using this formalization, we write the aggregated allocation as conditional expectation realizations; (iii) we apply the Levy's upward theorem to prove convergence. For the sake of clarity, the proof is organized in lemmas.

Formal notation and construction of the probability space. In each period, the outcomes of the idiosyncratic state lie in the finite set $\mathcal{S}$. We denote by $\left(\Pi_{s s^{\prime}}\right)$ the transition matrix for idiosyncratic histories (that be deduced from the transition matrix for productivity and for unemployment). The transition matrix being irreducible and finite as a consequence of Assumption B, it admits a unique stationary distribution $\left(\pi_{s}\right)$.

A steady-state infinite history can be seen as a one-sided infinite sequence of elements of $\mathcal{S}$. The space of idiosyncratic histories is thus $\mathcal{S}^{\infty}$ and a typical steady-state history will be denoted:

$$
s^{\infty}=\left(\ldots, s_{-N+1}\left(s^{\infty}\right), s_{-N+2}\left(s^{\infty}\right), \ldots, s_{-1}\left(s^{\infty}\right), s_{0}\left(s^{\infty}\right)\right),
$$

where $s_{-k}\left(s^{\infty}\right) \in \mathcal{S}$ for all $s^{\infty} \in \mathcal{S}^{\infty}$ and $k \geq 0$. Each $s_{-k}: \mathcal{S}^{\infty} \rightarrow \mathcal{S}$ is a coordinate function indicating the idiosyncratic state $k$ periods ago.

We focus on the so-called cylinder sets. A cylinder set $C_{N}(A)$ is defined as, for $N \geq 1$ 
and $A \subset \mathcal{S}^{N}$ :

$$
C_{N}(A) \equiv\left\{s^{\infty} \in \mathcal{S}^{\infty}:\left(s_{-N+1}\left(s^{\infty}\right), \ldots, s_{-1}\left(s^{\infty}\right), s_{0}\left(s^{\infty}\right)\right) \in A\right\}
$$

In words, $C_{N}(A)$ is the subset of histories whose truncation of length $N$ belongs to $A$.

We denote by $\mathcal{C}_{0}$ the set of all cylinder sets. We have our first lemma.

Lemma 1 The set $\mathcal{C}_{0}$ of all cylinder sets is an algebra.

Proof. $\emptyset=C_{1}(\emptyset) \in \mathcal{C}_{0} ; \mathcal{S}^{\infty}=C_{N}\left(\mathcal{S}^{N}\right) \in \mathcal{C}_{0}$; closed for complements since $\mathcal{S}^{\infty} \backslash C_{N}(A)=$ $C_{N}\left(S^{N} \backslash A\right)$; closed for finite unions. The last point is worth showing more carefully. Let $N \leq M, A \subset \mathcal{S}^{N}$ and $B \subset \mathcal{S}^{N}$. Note that:

$$
\begin{aligned}
C_{N}(A) & =\left\{s^{\infty} \in \mathcal{S}^{\infty}:\left(s_{-N+1}\left(s^{\infty}\right), \ldots, s_{0}\left(s^{\infty}\right)\right) \in A\right\}, \\
& =\left\{s^{\infty} \in \mathcal{S}^{\infty}:\left(s_{-M+1}\left(s^{\infty}\right), \ldots, s_{-N+1}\left(s^{\infty}\right), \ldots, s_{0}\left(s^{\infty}\right)\right) \in \mathcal{S}^{M-N} \times A\right\}, \\
& =C_{M}\left(\mathcal{S}^{M-N} \times A\right),
\end{aligned}
$$

where the first equality is the definition of $C_{N}(A)$, the second is an equivalent rewriting of the set definition (some coordinates prior to $N$ belong to $\mathcal{S}$ ), the third equality is the definition of $C_{M}\left(\mathcal{S}^{M-N} \times A\right)$. Using (54), we deduce $C_{N}(A) \cup C_{M}(B)=C_{M}\left(\mathcal{S}^{M-N} \times\right.$ $A) \cup C_{M}(B)$ and finally:

$$
C_{N}(A) \cup C_{M}(B)=C_{M}\left(\left(\mathcal{S}^{M-N} \times A\right) \cup B\right),
$$

which belongs to $\mathcal{C}_{0}$ and shows that $\mathcal{C}_{0}$ is closed for finite unions.

We now define the set function $\mu_{\infty}: \mathcal{C}_{0} \rightarrow \mathbb{R}$ from the matrix $\left(\Pi_{s s^{\prime}}\right)$ and the vector $\left(\pi_{s}\right):$

$$
\mu_{\infty}\left(C_{N}(A)\right)=\sum_{\left(s_{-N+1}, \ldots, s_{0}\right) \in A} \pi_{s_{-N+1}} \Pi_{s_{-N+1} s_{-N+2}} \ldots \Pi_{s_{-1} s_{0}} .
$$

Note that when $N=1$, we have $\mu_{\infty}\left(C_{1}\left(A_{1}\right)\right)=\sum_{s_{0} \in A_{1}} \pi_{s_{0}}$, where $A_{1} \subset \mathcal{S}$.

Lemma 2 The set function $\mu_{\infty}$ is a pre-measure on $\mathcal{C}_{0}$.

Proof. We need to verify three properties for $\mu_{\infty}$ to be a pre-measure on $\mathcal{C}_{0}$ : (i) welldefined, (ii) (countably) additive, (iii) $\mu_{\infty}\left(\mathcal{S}^{\infty}\right)=1$. Point (i) is related to the fact that cylinders may have non-unique representations, in particular $C_{N}(A)=C_{N}\left(\mathcal{S}^{M-N} \times A\right)$ 
for $M \geq N$. We need to check that $\mu_{\infty}\left(C_{N}(A)\right)=\mu_{\infty}\left(C_{N}\left(\mathcal{S}^{M-N} \times A\right)\right)$ for all $M \geq N$. For $M=N+1$, we have:

$$
\begin{aligned}
\mu_{\infty}\left(C_{M}(\mathcal{S} \times A)\right) & =\sum_{s_{-N} \in \mathcal{S},\left(s_{-N+1}, \ldots, s_{0}\right) \in A} \pi_{s_{-N}} \Pi_{s_{-N} s_{-N+1}} \Pi_{s_{-N+1} s_{-N+2}} \ldots \Pi_{s_{-1} s_{0}}, \\
& =\sum_{\left(s_{-N+1}, \ldots, s_{0}\right) \in A}\left[\sum_{s_{-N} \in \mathcal{S}} \pi_{s_{-N}} \Pi_{s_{-N} s_{-N+1}}\right] \Pi_{s_{-N+1} s_{-N+2}} \ldots \Pi_{s_{-1} s_{0}}, \\
& =\sum_{\left(s_{-N+1}, \ldots, s_{0}\right) \in A} \pi_{s_{-N+1}} \Pi_{s_{-N+1} s_{-N+2}} \ldots \Pi_{s_{-1} s_{0}}, \\
& =\mu_{\infty}\left(C_{N}(A)\right),
\end{aligned}
$$

where the first equality uses the definition of $\mu_{\infty}$ on $\mathcal{C}_{0}$, the second is a rearrangement of finite sums, the third uses the fact that $\pi$ is stationary $\left(\sum_{s \in \mathcal{S}} \pi_{s} \Pi_{s s^{\prime}}=\pi_{s^{\prime}}\right)$, and the last uses the definition of $\mu_{\infty}$ again. The proof for any $M \geq N$ can be completed by induction using the same lines. This shows point (i).

For point (ii), we consider two disjoint cylinders, $C_{N}(A)$ and $C_{M}(B)\left(M \geq N, A \subset \mathcal{S}^{N}\right.$ and $\left.B \subset \mathcal{S}^{M}\right)$. Since both cylinders are disjoint, then $\mathcal{S}^{M-N} \times A$ and $B$ must be disjoint too. We deduce that:

$$
\begin{aligned}
\mu_{\infty}\left(C_{N}(A) \cup C_{N}(B)\right)= & \mu_{\infty}\left(C_{M}\left(\left(\mathcal{S}^{M-N} \times A\right) \cup B\right)\right. \\
& =\sum_{\left(s_{-M+1}, \ldots, s_{0}\right) \in\left(\mathcal{S}^{M-N} \times A\right) \cup B} \pi_{s_{-M}} \Pi_{s_{-M} s_{-M+1}} \ldots \Pi_{s_{-1} s_{0}}, \\
& =\sum_{\left(s_{-M+1}, \ldots, s_{0}\right) \in\left(\mathcal{S}^{M-N} \times A\right)} \pi_{s_{-M} \Pi_{s_{-M} s_{-M+1}} \ldots \Pi_{s_{-1} s_{0}}} \\
& +\sum_{\left(s_{-M+1}, \ldots, s_{0}\right) \in B} \pi_{s_{-M}} \Pi_{s_{-M} s_{-M+1}} \ldots \Pi_{s_{-1} s_{0}}, \\
& =\mu_{\infty}\left(C_{M}\left(\mathcal{S}^{M-N} \times A\right)\right)+\mu_{\infty}\left(C_{N}(B)\right),
\end{aligned}
$$

where the first equality uses (55), the second the definition of $\mu_{\infty}$, the third the property that $\mathcal{S}^{M-N} \times A$ and $B$ are disjoint, the fourth the definition of $\mu_{\infty}$ again (twice). This proves that $\mu_{\infty}$ is finitely additive. Finally, for point (iii), note that, for any $N \geq 1$, we have using (56) iteratively, $\mu_{\infty}\left(C_{N}\left(\mathcal{S}^{N}\right)\right)=1$, which proves that $\mu_{\infty}\left(\mathcal{S}^{\infty}\right)=1$.

All these elements prove that $\mu_{\infty}$ is a finitely additive probability measure on the algebra $\mathcal{C}_{0}$. However, we know that any finitely additive probability measure on the cylinder 
algebra in $\mathcal{S}^{\infty}$ is countably additive (see Billingsley 2012, Theorem 2.3) and conclude that $\mu_{\infty}$ is a countably additive probability measure on $\mathcal{C}_{0}$. So, $\mu_{\infty}$ is a pre-measure on $\mathcal{C}_{0}$.

We denote by $\mathcal{F}=\sigma\left(\mathcal{C}_{0}\right)$ the cylindrical $\sigma$-algebra generated by $\mathcal{C}_{0}$.

Lemma 3 The triplet $\left(\mathcal{S}^{\infty}, \mathcal{F}, \mu_{\infty}\right)$ is a probability space.

Proof. We use the Hahn-Kolmogorov theorem (which is a simple version of the Carathéodory's extension theorem, see Billingsley 2012, Theorem 3.1), stating that: (i) any pre-measure defined on an algebra $A$ extents to a measure defined on the sigma-algebra generated by $A$; (ii) the restriction of the extended measure to $A$ coindices with the initial measure; (iii) the extended measure is unique when the initial measure is $\sigma$-finite. The theorem directly applies in our framework to the initial measure $\mu_{\infty}$ defined on the algebra $\mathcal{C}_{0}$. Since $\mu_{\infty}$ is finite $\left(\mu_{\infty}\left(\mathcal{S}^{\infty}\right)=1\right)$, hence $\sigma$-finite, the extension is unique. The unique extension of $\mu_{\infty}$ to $\mathcal{F}$ is also denoted (with a slight abuse of notation) $\mu_{\infty}$. The triplet $\left(\mathcal{S}^{\infty}, \mathcal{F}, \mu_{\infty}\right)$ is thus a probability space.

Aggregated allocation as conditional expectations. We define, for every $N \geq 1$, $\mathcal{F}_{N}=\sigma\left(\bigcup_{A \subset \mathcal{S}^{N}} C_{N}(A)\right)$ the $\sigma$-algebra generated by cylinder sets of ranks $N$.

Lemma 4 The sequence $\left(\mathcal{F}_{N}\right)_{N \geq 1}$ is a filtration of $\mathcal{F}$.

Proof. Using property (54), we deduce that $\bigcup_{A \subset \mathcal{S}^{N}} C_{N}(A)=\bigcup_{1 \leq k \leq N} \bigcup_{A \subset \mathcal{S}^{k}} C_{k}(A)$ ( $\subset$ is direct, while $\supset$ comes from (54) which implies that for $k \leq N, \bigcup_{A \subset \mathcal{S}^{k}} C_{k}(A)=$ $\left.\bigcup_{A \subset \mathcal{S}^{k}} C_{N}\left(\mathcal{S}^{N-k} \times A\right) \subset \bigcup_{A \subset \mathcal{S}^{N}} C_{N}(A)\right)$. We deduce that $\mathcal{F}_{N}=\sigma\left(\bigcup_{1 \leq k \leq N} \bigcup_{A \subset \mathcal{S}^{k}} C_{k}(A)\right)$. With this last notation, it is clear that the sequence of $\sigma$-algebras is increasing in the sense of inclusion: $\mathcal{F}_{N} \subset \mathcal{F}_{N+1}$ for any $N$ and we also have $\mathcal{F}_{N} \rightarrow_{N} \mathcal{F}_{\infty}=\mathcal{F}$ (since $\mathcal{F}$ is exactly defined as $\left.\mathcal{F}_{\infty}\right)$. So, $\left(\mathcal{F}_{N}\right)_{N \geq 1}$ is a filtration of $\mathcal{F}$.

Lemma 5 Let $N>0, s^{N} \in \mathcal{S}^{N}$, and $X$ be an integrable random variable. Define $X_{s^{N}}$ as the average value of $X$ over the cylinder set $C_{N}\left(\left\{s^{N}\right\}\right)$ :

$$
X_{s^{N}} \equiv \frac{1}{\mu_{\infty}\left(C_{N}\left(\left\{s^{N}\right\}\right)\right)} \int_{s^{\infty} \in C_{N}\left(\left\{s^{N}\right\}\right)} X\left(s^{\infty}\right) \mu_{\infty}\left(d s^{\infty}\right)
$$

We then have:

$$
X_{s^{N}}=\mathbb{E}\left[X \mid \mathcal{F}_{N}\right]_{s^{\infty}}, s^{\infty} \in C_{N}\left(\left\{s^{N}\right\}\right) .
$$


Proof. We will use the total expectation theorem. Let $\left(E_{1}, \ldots, E_{p}\right)$ be a partition of $\mathcal{S}^{\infty}$ that generates the sub $\sigma$-algebra $\mathcal{G} \subset \mathcal{F}$ (with $\mu_{\infty}\left(E_{i}\right)>0$ ), then we have for any integrable random variable $X$ :

$$
\begin{aligned}
\mathbb{E}[X \mid \mathcal{G}]_{s^{\infty}} & =\sum_{i=1}^{p} \mathbb{E}\left[X \mid E_{i}\right] 1_{s^{\infty} \in E_{i}}, \\
\text { where: } \mathbb{E}\left[X \mid E_{i}\right] & =\frac{1}{\mu_{\infty}\left(E_{i}\right)} \int_{s^{\infty} \in E_{i}} X\left(s^{\infty}\right) \mu_{\infty}\left(d s^{\infty}\right) .
\end{aligned}
$$

Let $N>0$. We apply this theorem to $\left(C_{N}\left(\left\{s^{N}\right\}\right)\right)_{s^{N} \in \mathcal{S}^{N}}$, where $C_{N}\left(\left\{s^{N}\right\}\right)$ is a thin cylinder corresponding to the histories $s^{\infty}$ whose realizations in the last $N$ periods is $s^{N}$ (see equation (53)). We have to prove that $\left(C_{N}\left(\left\{s^{N}\right\}\right)\right)_{s^{N} \in \mathcal{S}^{N}}$ is a partition of $\mathcal{S}^{\infty}$. First, if $s_{1}^{N} \neq s_{2}^{N}$, then $C_{N}\left(\left\{s_{1}^{N}\right\}\right) \cap C_{N}\left(\left\{s_{2}^{N}\right\}\right)=\emptyset$ since a given history $s^{\infty}$ cannot have two different realizations $s_{1}^{N} \neq s_{2}^{N}$ over the last $N$ periods. Second, let $s^{\infty} \in \mathcal{S}^{\infty}$. It can be written as $s^{\infty}=\left(\ldots, s_{-N}, s_{-N+1}, \ldots, s_{0}\right)$, so $s^{\infty} \in C_{N}\left(\left\{\left(s_{-N}, s_{-N+1}, \ldots, s_{0}\right)\right\}\right.$ and $\mathcal{S}^{\infty}=\bigcup_{s^{N} \in \mathcal{S}^{N}} C_{N}\left(\left\{s^{N}\right\}\right)$. So, $\left(C_{N}\left(\left\{s^{N}\right\}\right)\right)_{s^{N} \in \mathcal{S}^{N}}$ is a finite partition of $\mathcal{S}^{\infty}$. Furthermore, $\mathcal{F}_{N}=\sigma\left(\bigcup_{s^{N} \in \mathcal{S}^{N}} C_{N}\left(\left\{s^{N}\right\}\right)\right)$. We thus deduce from (60) and (61), for an integrable random variable $X$ :

$$
\begin{aligned}
\mathbb{E}\left[X \mid \mathcal{F}_{N}\right]_{s^{\infty}} & =\sum_{s^{N} \in \mathcal{S}^{N}} \mathbb{E}\left[X \mid C_{N}\left(\left\{s^{N}\right\}\right)\right] 1_{s^{\infty} \in C_{N}\left(\left\{s^{N}\right\}\right),} \\
\mathbb{E}\left[X \mid C_{N}\left(\left\{s^{N}\right\}\right)\right] & =\frac{1}{\mu_{\infty}\left(C_{N}\left(\left\{s^{N}\right\}\right)\right)} \int_{s^{\infty} \in C_{N}\left(\left\{s^{N}\right\}\right)} X\left(s^{\infty}\right) \mu_{\infty}\left(d s^{\infty}\right) .
\end{aligned}
$$

Since $\mathcal{S}^{N}$ is a finite set and since with our previous notation, $S_{s^{N}}=\mu_{\infty}\left(C_{N}\left(\left\{s^{N}\right\}\right)\right)$ (see equation (22)), we can also rewrite equation (62) as:

$$
\mathbb{E}\left[X \mid C_{N}\left(\left\{s^{N}\right\}\right)\right]=\frac{1}{S_{s^{N}}} \sum_{s^{\infty} \in \mathcal{S}^{\infty} \mid\left(s_{-N+1}^{\infty}, \ldots, s_{0}^{\infty}\right)=s^{N}} X\left(s^{\infty}\right) \mu_{\infty}\left(s^{\infty}\right),
$$

which is exactly our aggregation equation (23). Finally, we deduce:

$$
X_{s^{N}}=\mathbb{E}\left[X \mid \mathcal{F}_{N}\right]_{s^{\infty}}, s^{\infty} \in C_{N}\left(\left\{s^{N}\right\}\right) .
$$

\section{Convergence of allocation.}


Lemma 6 For any integrable random variable $X$, we have:

$$
X_{s^{N}}=\mathbb{E}\left[X \mid \mathcal{F}_{N}\right]_{s^{\infty}} \rightarrow_{N} X_{s^{\infty}}, \text { a.s. }
$$

where $X_{s^{N}}$ is defined in (63).

Proof. We conclude the proof using the Levy's upward theorem. This theorem states for any filtration $\left(\mathcal{G}_{N}\right)_{N}$ of $\mathcal{F}$, we have for any integrable random variable $Y, \mathbb{E}\left[Y \mid \mathcal{G}_{N}\right]_{s} \rightarrow_{N}$ $\mathbb{E}\left[Y \mid \mathcal{G}_{\infty}\right]_{s^{\infty}}$, almost surely and in $L^{1}$. We apply this theorem to the filtration $\left(\mathcal{F}_{N}\right)_{N}$, for which $\mathcal{F}_{\infty}=\mathcal{F}$ and we deduce, for any integrable random variable $X$ :

$$
X_{s^{N}}=\mathbb{E}\left[X \mid \mathcal{F}_{N}\right]_{s^{\infty}} \rightarrow_{N} X_{s^{\infty}}, \text { a.s. }
$$

This convergence theorem can be applied to all terms of the tuple $\left(c_{s^{N}}, l_{s^{N}}, a_{s^{N}}, \nu_{s^{N}}\right)_{s^{N}}$, which can be assumed to be integrable following Assumption B and Açikgöz (2018). Lemma 6 concludes the first part of the proof.

\section{Convergence of the $\xi \mathrm{s}$.}

Lemma 7 We have:

$$
\xi_{s^{N}} \rightarrow_{N} 1, a . s .
$$

Proof. Using the convergence result of Lemma 6, we have $\xi_{s^{N}} \rightarrow \xi_{s^{\infty}}$, almost surely. We need to show that $\xi_{s^{\infty}}=1$, a.s. Using the continuity of $U_{c}$, passing to the limit in the aggregate Euler equation for consumption (26) yields:

$$
\xi_{s^{\infty}} U_{c}\left(c_{s} \infty, l_{s} \infty\right)=\beta(1+r) \int_{\tilde{s}^{\infty} \in \mathcal{S}^{\infty}} \xi_{\tilde{s}^{\infty}} U_{c}\left(c_{\tilde{s}^{\infty}}, l_{\tilde{s}^{\infty}}\right) \mu_{\infty}\left(d \tilde{s}^{\infty} \mid s^{\infty}\right)+\nu_{s^{\infty}},
$$

where $\mu_{\infty}\left(d \tilde{s}^{\infty} \mid s^{\infty}\right)$ denotes the conditional probability of having next-period history $\tilde{s}^{\infty}=\left(\ldots, \tilde{s}_{0}\right)$ conditional on having current history $s^{\infty}=\left(\ldots, s_{0}\right)$ (which is simply $\Pi_{s_{0} \tilde{s}_{0}}$ given our notation). By difference with the individual Euler equation, we have:

$$
\left(\xi_{s^{\infty}}-1\right) U_{c}\left(c_{s} \infty, l_{s^{\infty}}\right)=\beta(1+r) \int_{\tilde{s}^{\infty} \in \mathcal{S}^{\infty}}\left(\xi_{\tilde{s}^{\infty}}-1\right) U_{c}\left(c_{\tilde{s}^{\infty}}, l_{\tilde{s}^{\infty}}\right) \mu_{\infty}\left(d \tilde{s}^{\infty} \mid s^{\infty}\right),
$$

which holds a.s., or taking the absolute value:

$$
\left|\xi_{s^{\infty}}-1\right| U_{c}\left(c_{s} \infty, l_{s} \infty\right) \leq \beta(1+r) \int_{\tilde{s}^{\infty} \in \mathcal{S}^{\infty}}\left|\xi_{\tilde{s}^{\infty}}-1\right| U_{c}\left(c_{\tilde{s}^{\infty}}, l_{\tilde{s}^{\infty}}\right) \mu_{\infty}\left(d \tilde{s}^{\infty} \mid s^{\infty}\right) .
$$


We now sum over all histories $s^{\infty} \in \mathcal{S}^{\infty}$ the previous inequality (holding a.s.) and deduce using Bayes' law:

$$
0 \leq(1-\beta(1+r)) \int_{s^{\infty} \in \mathcal{S}^{\infty}}\left|\xi_{s^{\infty}}-1\right| U_{c}\left(c_{s} \infty, l_{s} \infty\right) \mu_{\infty}\left(d s^{\infty}\right) \leq 0
$$

where the left hand-side inequality comes from $\beta(1+r)<1$ and the positivity of the integral. We finally deduce from $U_{c}>0$ that $\left|\xi_{s^{\infty}}-1\right|=0$ a.s., and $\xi_{s^{\infty}}=1$ a.s.

Lemma 7 concludes the proof of Proposition 2.

\section{B.4 Proof of Proposition 3}

The proof is conducted in two steps. We first prove the following proposition, in which we do not impose the uniqueness of the solution to the original Ramsey problem. The proof can be found in Section B.4.1. The proof of Proposition 3 can be found in Section B.4.2.

Proposition 5 (Convergence of allocations) We consider a sequence of Ramsey equilibria $\left(\phi_{N}, \tau_{N}, r_{N}, w_{N},\left(a_{s^{N}}, c_{s^{N}}, l_{s^{N}}, \nu_{s^{N}}, \xi_{s^{N}}\right)_{s^{N} \in \mathcal{S}^{N}}\right)_{N \geq 0}$, such that:

1. for all $N$, there exists an original Bewley allocation $\left(a_{s^{\infty}, N}, c_{s}^{\infty}, N, l_{s^{\infty}, N}, \nu_{s^{\infty}, N}\right)_{s^{\infty} \in \mathcal{S}^{\infty}}$ such that the aggregation following equation (23) of $\left(c_{s^{\infty}, N}, l_{s^{\infty}, N}, a_{s^{\infty}, N}, \nu_{s^{\infty}, N}\right)_{s^{\infty} \in \mathcal{S} \infty}$ yields the aggregated allocation $\left(a_{s^{N}}, c_{s^{N}}, l_{s^{N}}, \nu_{s^{N}},\right)_{s^{N} \in \mathcal{S}^{N}}$;

2. for all $N$, the first-order equations characterizing the aggregated Ramsey equilibrium, (25)-(29) and (33)-(34), hold for $\left(\phi_{N}, r_{N}, w_{N},\left(a_{s^{N}}, c_{s^{N}}, l_{s^{N}}, \nu_{s^{N}}, \xi_{s^{N}}\right)_{s^{N} \in \mathcal{S}^{N}}\right)$;

3. there exist $\left(\left(1+\frac{1}{\varphi}\right) \frac{S_{e}}{S_{u}}+1\right)^{-1}<\underline{\tau}<\bar{\tau}<1$, such that for all $N: \underline{\tau} \leq \tau_{N} \leq \bar{\tau}$.

Then, there exists a subsequence $\left(N_{k}\right)_{k}$ such that:

1. the UI policy $\left(\phi_{N_{k}}, \tau_{N_{k}}\right)$ converges with $k$ to a limit $\left(\phi^{*}, \tau^{*}\right)$;

2. prices $\left(r_{N_{k}}, w_{N_{k}}\right)$ converges with $k$ to a limit $\left(r^{*}, w^{*}\right)$;

3. the allocation $\left(a_{s^{N_{k}}}, c_{s^{N_{k}}}, l_{s^{N_{k}}}, \nu_{s^{N_{k}}}\right)_{s^{N_{k}} \in \mathcal{S}^{N_{k}}}$ converges almost surely with $k$ to a limit $\left(a_{s^{\infty}}^{*}, c_{s^{\infty}}^{*}, l_{s^{\infty}}^{*}, \nu_{s^{\infty}}^{*}\right)_{s^{\infty} \in \mathcal{S}^{\infty}} ;$

4. the allocation $\left(a_{s^{\infty}}^{*}, c_{s^{\infty}}^{*}, l_{s^{\infty}}^{*}, \nu_{s^{\infty}}^{*}\right)_{s^{\infty} \in \mathcal{S}^{\infty}}$ with the UI policy $\left(\phi^{*}, \tau^{*}\right)$ and prices $\left(r^{*}, w^{*}\right)$ is a solution of the original Ramsey program of Section 3 (characterized by equations (6)-(14) and (18)-(19)); 
5. $\left(\xi_{s^{N_{k}}}\right)_{s^{N_{k}} \in \mathcal{S}^{N_{k}}} \longrightarrow_{k}(1)_{s^{\infty} \in \mathcal{S}^{\infty}}$, almost surely.

\section{B.4.1 Proof of Proposition 5}

For a given Ramsey allocation, we make the dependence in $\phi$ explicit. The sequence of Ramsey equilibria we consider in Proposition 5 will be denoted by the sequence $\left(\phi_{N}, \tau_{N}\left(\phi_{N}\right), r_{N}\left(\phi_{N}\right), w_{N}\left(\phi_{N}\right),\left(c_{s^{N}}\left(\phi_{N}\right), l_{s^{N}}\left(\phi_{N}\right), a_{s^{N}}\left(\phi_{N}\right), \nu_{s^{N}}\left(\phi_{N}\right), \xi_{s^{N}}\left(\phi_{N}\right)\right)_{s^{N} \in \mathcal{S}^{N}}\right)_{N \geq 0}$.

This sequence verifies:

1. for all $N$, there exists an original Bewley allocation $\left(c_{s} \infty\left(\phi_{N}\right), l_{s} \infty\left(\phi_{N}\right), a_{s} \infty\left(\phi_{N}\right)\right.$, $\left.\nu_{s^{\infty}}\left(\phi_{N}\right)\right)$ corresponding to UI policy $\left(\phi_{N}, \tau_{N}\left(\phi_{N}\right)\right)$, and prices $\left(r_{N}\left(\phi_{N}\right), w_{N}\left(\phi_{N}\right)\right)$, such that the aggregation of this original allocation yields the aggregated allocation denoted by $\left(a_{s^{N}}\left(\phi_{N}\right), c_{s^{N}}\left(\phi_{N}\right), l_{s^{N}}\left(\phi_{N}\right), \nu_{s^{N}}\left(\phi_{N}\right), \xi_{s^{N}}\left(\phi_{N}\right)\right)_{s^{N} \in \mathcal{S}^{N}}$;

2. the set of equations, characterizing the aggregated Ramsey equilibrium, (25)-(29) and (33)-(34) holds;

3. there exist $\left(\left(1+\frac{1}{\varphi}\right) \frac{S_{e}}{S_{u}}+1\right)^{-1}<\underline{\tau}<\bar{\tau}<1$, such that for all $N: \underline{\tau} \leq \tau_{N} \leq \bar{\tau}$, and markets clear.

Note that point 3 implies the existence of $\underline{\phi}, \bar{\phi}>0$, such that $\phi_{N} \in[\underline{\phi}, \bar{\phi}]$ for all $N$.

Preliminary remarks and notation. First, for a given UI policy $(\phi, \tau)$, the existence of a Bewley equilibrium is well understood. In particular, Assumption B (Section 4.1.4) guarantees that the structure of the proof of Açikgöz (2018) (who also considers a GHH utility function) can be applied in our environment. As a consequence, we do not prove here the equilibrium existence for a given UI policy $(\phi, \tau)$. Second, the proof consists in showing that $\left(\phi_{N}, \tau_{N}\left(\phi_{N}\right), r_{N}\left(\phi_{N}\right), w_{N}\left(\phi_{N}\right),\left(c_{s^{N}}\left(\phi_{N}\right), l_{s^{N}}\left(\phi_{N}\right), a_{s^{N}}\left(\phi_{N}\right), \nu_{s^{N}}\left(\phi_{N}\right), \xi_{s^{N}}\left(\phi_{N}\right)\right)_{s^{N} \in \mathcal{S}^{N}}\right)_{N \geq 0}$ belongs to a compact set. To do so, we will start with proving that the sequence of equilibrium prices $\left(r_{N}\left(\phi_{N}\right)\right)_{N}$ belongs to a compact set $[\underline{\rho}, \bar{\rho}]$ where $-1<\underline{\rho} \leq \bar{\rho}<(1+\beta)^{-1}-1$. The proof involves two intermediary results (Lemmas 9 and 10), which generalize two results of Açikgöz (2018) to all $\phi \in[\underline{\phi}, \bar{\phi}]$.

Note that from factor prices (3) and the labor Euler equation (9), we deduce the 
demand for capital given the interest rate $r, K(r)$, has the following expression:

$$
\begin{aligned}
K(r) & =(1-\alpha) \kappa_{L}^{1-\alpha+\alpha \varphi}\left(\frac{r+\delta}{\alpha}\right)^{-\frac{1+\alpha \varphi}{1-\alpha}}, \\
& =(1-\alpha)^{-\frac{1}{\alpha}} \kappa_{L} w(r)^{\varphi+\frac{1}{\alpha}} \\
\text { where: } \kappa_{L} & =\chi^{\varphi}(1-\tau)^{\varphi} S_{e} \sum_{y \in \mathcal{Y}} S_{y} y^{1+\varphi}
\end{aligned}
$$

where $w(r)$ is the wage as a function of the interest rate, from the first-order conditions of the firm.

We state a preliminary lemma.

Lemma 8 The demand for capital given interest rate $r, K(r)$, verifies for all $r \in[0, \bar{r})$ :

$$
K(r) \leq K(0)<\infty
$$

Proof. Since $\alpha \in(0,1)$, we deduce from (64) that: $K(\cdot)$ decreases with $r ; 0<K(r)<\infty$ for all $r \in(-\delta, \bar{r}) ; \lim _{r \rightarrow \delta} K(r)=\infty$. In particular, we have $K(r) \leq K(0)<\infty$ for all $r \in[0, \bar{r})$.

We denote $\bar{r}=(1+\beta)^{-1}-1$. We define the quantity $X D(r, \phi)$ as the excess demand function, as a function of the real interest rate $r$ and the replacement rate $\phi$. Formally, $X D(r, \phi)=K(r)-A(r, \phi)$, where $A(r, \phi)$ the aggregate asset demand of an original Bewley model, with interest rate $r$ and UI policy $(\phi, \tau(\phi))$.

Lemma 9 There exists a continuous function $f:(0, \bar{r}) \mapsto f(r)$, such that: (i) $f$ is independent of $\phi$; (ii) $\lim _{r \rightarrow \bar{r}} f(r)=1$; and (iii) for any $L>0, r \in(0, \bar{r}), \phi \in[\phi, \bar{\phi}]$, we have $A(r, \phi) \geq L f(r)$.

Proof. Our proof builds on the proof of Açikgöz (2018, Proof of Lemma 1). Using Proposition 5 in Açikgöz (2018), we know that there exists a unique stationary distribution defined on the space asset holdings $\times$ idiosyncratic shocks. We denote it $\mu(a, s ; r, \phi)$ to highlight the dependence in $r$ and $\phi$ ( $a$ is asset holding and $s$ idiosyncratic state). We also denote by $C$ the "aggregate consumption":

$$
C(a, s ; r, \phi)=c(a, s ; r, \phi)-\chi^{-1} \frac{\hat{l}(a, s ; r, \phi)^{1+1 / \varphi}}{1+1 / \varphi}
$$


The integration of consumption Euler equations yields:

$$
\begin{gathered}
\int_{(a, s)} u^{\prime}(C(a, s ; r, \phi)) d \mu(\cdot ; r, \phi) \\
=\beta(1+r) \int_{(a, s)} \sum_{s^{\prime} \in \mathcal{S}} \Pi_{s s^{\prime}} u^{\prime}\left(C\left(g_{a}(a, s ; r, \phi), s^{\prime} ; r, \phi\right)\right) d \mu(\cdot ; r, \phi)+\int_{(a, s)} \nu(a, s ; r, \phi) d \mu(\cdot ; r, \phi),
\end{gathered}
$$

where $g_{a}(a, s ; r, \phi)$ is the saving policy function. We need to show that marginal utilities are finite. The budget constraint (6) implies at the steady-state:

$$
c=r a+\left((1-\tau) 1_{e(s)=e}+\phi 1_{e(s)=u}\right) l y(s) w
$$

where $e(s)$ and $y(s)$ are the employment and productivity statuses in state $s$. We can simplify the expression of aggregate consumption $C$ using the labor Euler equation (9):

$$
\begin{aligned}
C(a, s ; r, \phi)= & r a+\tilde{w}(s ; r, \phi) \\
\text { with: } \tilde{w}(s ; r, \phi)= & \left(\frac{1-\tau}{1+\varphi} 1_{e(s)=e}+\left(\left(\left(1+\frac{1}{\varphi}\right) \frac{S_{e}}{S_{u}}+1\right) \tau-1\right) \frac{1_{e(s)=u}}{1+1 / \varphi}\right) \\
& \times \chi^{\varphi}(1-\tau)^{\varphi} w^{\varphi+1} y(s)^{\varphi+1} .
\end{aligned}
$$

Let define the constants $\underline{\kappa}_{C}$ and $\bar{\kappa}_{C}$ as follows:

$$
\begin{aligned}
& \underline{\kappa}_{C}=\min \left(\frac{1-\bar{\tau}}{1+\varphi},\left(\left(\left(1+\frac{1}{\varphi}\right) \frac{S_{e}}{S_{u}}+1\right) \underline{\tau}-1\right) \frac{1}{1+1 / \varphi}\right) \chi^{\varphi}(1-\bar{\tau})^{\varphi}>0 \\
& \bar{\kappa}_{C}=\max \left(\frac{1-\underline{\tau}}{1+\varphi},\left(\left(\left(1+\frac{1}{\varphi}\right) \frac{S_{e}}{S_{u}}+1\right) \bar{\tau}-1\right) \frac{1}{1+1 / \varphi}\right) \chi^{\varphi}(1-\underline{\tau})^{\varphi}>0
\end{aligned}
$$

where the inequalities come from $1>\bar{\tau} \geq \tau \geq \underline{\tau}>\left(\left(1+\frac{1}{\varphi}\right) \frac{S_{e}}{S_{u}}+1\right)^{-1}$. We deduce from (67) and (69) that we have for all $a, s, \phi$ :

$$
\bar{\kappa}_{C} w^{\varphi+1} \bar{y}^{\varphi+1}+r a \geq C(a, s ; r, \phi) \geq \underline{\kappa}_{C} w^{\varphi+1} \underline{y}^{\varphi+1}>0
$$

where $\underline{y}$ and $\bar{y}$ are the worst and best productivity levels. This guarantees that marginal utilities in (66) are finite and strictly positive. Furthermore, the support of $\mu(\cdot ; r, \phi)$ is compact (Açikgöz, 2018, Proposition 4), so we can simplify the expression of (66) as follows:

$$
(1-\beta(1+r)) \int_{(a, s)} u^{\prime}(C(a, s ; r, \phi)) d \mu(\cdot ; r, \phi)=\int_{(a, s)} \nu(a, s ; r) d \mu(\cdot ; r, \phi) .
$$


Now, note that:

$$
\int_{(a, s)} \nu(a, s ; r) d \mu(\cdot ; r, \phi) \geq \nu(0, \underline{s} ; r, \phi) \mu(\{(0, \underline{s})\} ; r, \phi),
$$

where $\underline{s}$ is the "worst" idiosyncratic state (i.e., $(\underline{y}, e)$ or $(\underline{y}, u)$ depending on the value of $\tau)$. From Açikgöz (2018, Proof of Lemma 1), we know that there exists $q \in(0,1]$ such that for any $L>0$ :

$$
\begin{aligned}
\mu(\{(0, \underline{s})\} ; r, \phi) & \geq \mu_{L}(r, \phi) q, \\
\text { where: } \mu_{L}(r, \phi) & =\mu([0, L] \times \mathcal{S} ; r, \phi) .
\end{aligned}
$$

This result holds for a given $\phi$ and comes from the fact that thanks to Point 4 in Assumption B, Section 4.1 .4 , any agent can reach the state $(0, \underline{s})$ (i.e., zero asset in worst state, be it $(\underline{y}, e)$ or $(\underline{y}, u))$ in at most $m<\infty$ periods with strictly positive probability $q$ - the same $q$ as in (73)). We now turn to the term $\nu(0, \underline{s} ; r, \phi)$ in (72). Using the consumption Euler equation:

$$
\begin{aligned}
\nu(0, \underline{s} ; r, \phi) & =u^{\prime}(C(0, \underline{s} ; r, \phi))-\beta(1+r) \mathbb{E}\left[u^{\prime}\left(C^{\prime}\right) \mid(0, \underline{s})\right] \\
& \geq u^{\prime}(C(0, \underline{s} ; r, \phi))-\mathbb{E}\left[u^{\prime}\left(C^{\prime}\right) \mid(0, \underline{s})\right]
\end{aligned}
$$

using $\beta(1+r)<1$. Furthermore using (67)-(68) and the same technique as in Açikgöz (2018, Proposition 2), we can prove that:

$$
C(a, s ; r, \phi) \geq r a+\frac{r}{1+r} \tilde{w}(s ; r, \phi)+\frac{1}{1+r} \tilde{w}(\underline{s} ; r, \phi),
$$

By construction, we have $\tilde{w}\left(\left(e, y^{\prime}\right) ; r, \phi\right)>\tilde{w}((e, y) ; r, \phi)$ and $\tilde{w}\left(\left(u, y^{\prime}\right) ; r, \phi\right)>\tilde{w}((u, y) ; r, \phi)$ iff $y^{\prime}>y$. In particular, $\tilde{w}\left(\left(e(\underline{s}), y^{\prime}\right) ; r, \phi\right)>\tilde{w}((e(\underline{s}), y) ; r, \phi)$ iff $y^{\prime}>y$. With this notation, we also have: $C(0, \underline{s} ; r, \phi)=\tilde{w}(\underline{s} ; r, \phi)$. Plugging this and (75) into (74) yields with $u$ being concave:

$$
\begin{aligned}
\nu(0, \underline{s} ; r, \phi) & \geq u^{\prime}(\tilde{w}(\underline{s} ; r, \phi))-\mathbb{E}\left[u^{\prime}\left(r a^{\prime}+\frac{r}{1+r} \tilde{w}\left(s^{\prime} ; r, \phi\right)+\frac{1}{1+r} \tilde{w}(\underline{s} ; r, \phi)\right) \mid(0, \underline{s})\right], \\
& \geq u^{\prime}(\tilde{w}(\underline{s} ; r, \phi))-\mathbb{E}\left[u^{\prime}\left(\frac{r}{1+r} \tilde{w}\left(s^{\prime} ; r, \phi\right)+\frac{1}{1+r} \tilde{w}(\underline{s} ; r, \phi)\right) \mid \underline{s}\right] \\
& \geq u^{\prime}\left(\tilde{w}\left(\underline{s} ; r, \phi_{m}\right)\right)-\mathbb{E}\left[u^{\prime}\left(\frac{r}{1+r} \tilde{w}\left(s^{\prime} ; r, \phi_{m}\right)+\frac{1}{1+r} \tilde{w}\left(\underline{s} ; r, \phi_{m}\right)\right) \mid \underline{s}\right] .
\end{aligned}
$$

The second inequality holds for all $\phi$ and comes from $a^{\prime} \geq 0, u$ concave and the independence 
in asset holdings. The third inequality is independent of $\phi$ and uses continuity of $\tilde{w}$ and $u^{\prime}$ over the compact set $[\underline{\phi}, \bar{\phi}]$ to guarantee the existence of such a $\phi_{m} \in[\underline{\phi}, \bar{\phi}]$. Finally, it can be observed that the term $u^{\prime}\left(\frac{r}{1+r} \tilde{w}\left(s^{\prime} ; r, \phi_{m}\right)+\frac{1}{1+r} \tilde{w}\left(\underline{s} ; r, \phi_{m}\right)\right)$ is equal to $u^{\prime}\left(\tilde{w}\left(\underline{s} ; r, \phi_{m}\right)\right)$ iff $s^{\prime}=\underline{s}$ and strictly smaller than $u^{\prime}\left(\tilde{w}\left(\underline{s} ; r, \phi_{m}\right)\right)$ for all $s^{\prime}=\left(e(\underline{s}), y^{\prime}\right)$ such that $y^{\prime}>\underline{y}$. So using Point 3 in Assumption B, we know that there exists $\underline{\nu}$, independent of $\phi$, such that.

$$
\nu(0, \underline{s} ; r, \phi) \geq \underline{\nu}>0
$$

which means that the agent with no asset and in the worst state $\underline{s}$ is always creditconstrained.

Putting together equations (71), (72), (73), and (76) finally yields:

$$
\begin{aligned}
\mu_{L}(r, \phi) q \underline{\nu} & \leq(1-\beta(1+r)) \int_{(a, s)} u^{\prime}(C(a, s ; r, \phi)) d \mu(\cdot ; r, \phi), \\
& \leq(1-\beta(1+r)) u^{\prime}(\tilde{w}(\underline{s} ; r, \phi)), \\
& \leq(1-\beta(1+r)) u^{\prime}\left(\tilde{w}\left(\underline{s} ; r, \tilde{\phi}_{m}\right)\right),
\end{aligned}
$$

where the first inequality uses (75), $a \geq 0$, and $\underline{s}$ being the worst state, and the second inequality uses the continuity of $\phi \mapsto \tilde{w}(\underline{s} ; r, \phi)$ on $[\underline{\phi}, \bar{\phi}]$ to ensure the existence of $\tilde{\phi}_{m} \in[\underline{\phi}, \bar{\phi}]$ (actually, we have $\tilde{\phi}_{m}=\underline{\phi}$ or $\tilde{\phi}_{m}=\bar{\phi}$, depending on whether the worst state features employment or unemployment, i.e. whether $e(\underline{s})=e$ or $e(\underline{s})=u$ ). This implies that:

$$
0 \leq \mu_{L}(r, \phi) \leq(1-\beta(1+r)) u^{\prime}\left(\tilde{w}\left(\underline{s} ; r, \tilde{\phi}_{m}\right)\right)
$$

where the upper bound is independent of $\phi$. Now, using Markov inequality we have:

$$
1-\mu_{L}(r, \phi)=P(a>L ; r, \phi) \leq \frac{1}{L} A(r, \phi),
$$

which implies with (77) that for all $L>0, r \in(0, \bar{r})$, and $\phi \in[\underline{\phi}, \bar{\phi}]$ :

$$
A(r, \phi) \geq L f(r)
$$

where: $f(r)=1-(1-\beta(1+r)) u^{\prime}\left(\tilde{w}\left(\underline{s} ; r, \tilde{\phi}_{m}\right)\right)$.

Note that $f(\cdot)$ is independent of $\phi$, continuous, and verifies $\lim _{r \rightarrow \bar{r}} f(r)=1$.

A direct corollary of Lemma 9 (and of inequality (78)) is that $\lim _{r \rightarrow \bar{r}} A(r, \phi)=\infty$ for 
all $\phi \in[\underline{\phi}, \bar{\phi}]$.

Lemma 10 The correspondence $\phi \in[\underline{\phi}, \bar{\phi}] \mapsto \arg \sup _{r \in[\underline{\rho}, \bar{\rho}]}\left\{-X D(r, \phi)^{2}\right\}$ (associating $\phi$ to an equilibrium interest rate) is upper-hemicontinuous and compact-valued.

Proof. We proceed in three steps. For each step, we prove an intermediary result that is stated in italic. For the sake of conciseness, we do not introduce formal lemma.

1. We prove that there exists $\bar{\rho} \in[0, \bar{r})$, such that for all $\phi \in[\underline{\phi}, \bar{\phi}], X D(\bar{\rho}, \phi)<0$. Inequality (78) with $L=2 K(0)+1$ implies that there exists $\bar{\rho} \in[0, \bar{r}$ ) (independent of $\phi)$, such that:

$$
\forall \phi \in[\underline{\phi}, \bar{\phi}], A(\bar{\rho}, \phi) \geq(2 K(0)+2) \frac{1}{2}>K(0)
$$

Since $K(0) \geq K(r)$ for all $r>0$, we deduce that for all $\phi \in[\underline{\phi}, \bar{\phi}]$ :

$$
X D(\bar{\rho}, \phi)=K(\bar{\rho})-A(\bar{\rho}, \phi)<0 .
$$

2. We prove that there exists $\underline{\rho} \in(-\delta, 0]$, such that for all $\phi \in[\underline{\phi}, \bar{\phi}], X D(\underline{\rho}, \phi)>0$. The individual budget constraint (6) and labor Euler equations (9) imply, when $r<0: a \leq-\frac{1}{r}(1+\bar{\phi}) \chi^{\varphi} \bar{y}^{\varphi+1} w^{\varphi+1}$ or, after integration:

$$
X D(r, \phi) \geq K(r)\left(1+\frac{1+\bar{\phi}}{r} \chi^{\varphi} \bar{y}^{\varphi+1} \frac{w(r)^{\varphi+1}}{K(r)}\right) .
$$

Using (64) and (65), we deduce that $K(r) \rightarrow_{r \rightarrow-\delta} \infty$ and $\frac{w(r)^{\varphi+1}}{K(r)} \rightarrow_{r \rightarrow-\delta} 0$. We deduce that $X D(r, \phi)$ is bounded from below by a function that diverges to $\infty$, independently of $\phi$. We deduce, since $X D(r, \phi)<\infty$ for all $r \in(-\delta, 0]$ and all $\phi \in[\underline{\phi}, \bar{\phi}]$ that there exists $\underline{\rho} \in(-\delta, 0]$ (independent of $\phi$ ), such that:

$$
\forall \phi \in[\underline{\phi}, \bar{\phi}], X D(\underline{\rho}, \phi)>0
$$

3. We prove that $R: \phi \in[\underline{\phi}, \bar{\phi}] \mapsto \arg \sup _{r \in[\underline{\rho}, \bar{\rho}]}\left\{-X D(r, \phi)^{2}\right\}$ is upper-hemicontinuous, as well as nonempty and compact-valued. Açikgöz (2018, Lemma G.2) can be extended in our setup to show that we can find a uniform upper bound on asset holdings for all $r \in[\underline{\rho}, \bar{\rho}]$ and all $\phi \in[\underline{\phi}, \bar{\phi}]$. Moreover, it is known that the policy functions are continuous in $(r, w, \phi)$ over any compact subset of $\{(r, w, \phi) \mid \beta \in$ 
$(0,1), \beta(1+r)<1, w>0, \phi \in[\underline{\phi}, \bar{\phi}]\}$ (see Açikgöz, 2018, Lemma G.3 for a proof in a related environment). We can thus apply Stokey et al. (1989, Theorem 12.13) that implies that the stationary distribution $\mu(\cdot ; r, \phi)$ is continuous (in the sense of the weak convergence) on $[\underline{\rho}, \bar{\rho}] \times[\underline{\phi}, \bar{\phi}]$. Because the support of measures (over asset space and idiosyncratic space) is uniformly bounded, the continuity in the sense of the weak convergence of $\mu(\cdot ; r, \phi)$ implies the continuity (in the usual sense) of $A(r, \phi)$ over $[\underline{\rho}, \bar{\rho}] \times[\underline{\phi}, \bar{\phi}]$. We deduce that $X D(r, \phi)$ is continuous over $[\underline{\rho}, \bar{\rho}] \times[\underline{\phi}, \bar{\phi}]$, while we already proved that: $X D(\bar{\rho}, \phi)<0<X D(\underline{\rho}, \phi)$. The intermediate value theorem guarantees that for all $[\underline{\phi}, \bar{\phi}]$, there exists $r_{\phi} \in[\underline{\rho}, \bar{\rho}]$, such that $X D\left(r_{\phi}, \phi\right)=0$. Since $-X D(r, \phi)^{2}$ is nonpositive, we deduce that $R(\phi)=\arg \max _{r \in[\underline{\rho}, \bar{\rho}]}\left\{-X D(r, \phi)^{2}\right\}$ is non empty. Furthermore, we can apply the Maximum theorem and obtain that $R(\cdot)$ is upper-hemicontinuous and compact-valued over $[\underline{\phi}, \bar{\phi}]$.

Conclusion of the proof. We prove a last lemma.

Lemma 11 We can extract a convergent subsequence of the sequence $\left(\phi_{N}, r_{N}\left(\phi_{N}\right)\right.$, $\left.\left(a_{s^{\infty}}\left(\phi_{N}\right)\right)_{s^{\infty} \in \mathcal{S}^{\infty}}\right)_{N \geq 0}$, such that:

$$
\phi_{N_{k}} \rightarrow_{N_{k}} \phi^{*}, r\left(\phi_{N_{k}}\right) \rightarrow_{N_{k}} r^{*}\left(\phi^{*}\right), \text { and }\left(a_{s^{\infty}}\left(\phi_{N_{k}}\right)\right)_{s^{\infty} \in \mathcal{S}^{\infty}} \rightarrow_{N_{k}, a . s .}\left(a_{s^{\infty}}^{*}\left(\phi^{*}\right)\right)_{s^{\infty} \in \mathcal{S}^{\infty}} .
$$

Proof. Lemma 10 implies that the joint sequence $\left(\phi_{N}, r_{N}\left(\phi_{N}\right)\right)_{N}$ belongs to a compact set: $\forall N,\left(\phi_{N}, r_{N}\left(\phi_{N}\right)\right) \in[\underline{\phi}, \bar{\phi}] \times[\underline{\rho}, \bar{\rho}]$. We need to show that $\left(a_{s^{\infty}}\left(\phi_{N}\right)\right)_{s^{\infty} \in \mathcal{S}^{\infty}}$ also lies in a compact set. We again use the fact that there is a uniform upper bound on asset holdings, such that asset holdings can be defined over compact set $\mathcal{A}$, and that the policy functions are continuous in $(a, r, w, \phi)$. Then, for the saving policy function, denoted as $g_{a}(a, r, w, \phi)$, the correspondence $(a, \phi) \in \mathcal{A} \times[\underline{\phi}, \bar{\phi}] \mapsto\left\{g_{a}\left(a, r_{\phi}, w\left(r_{\phi}\right), \phi\right): r_{\phi} \in R(\phi) \subset[\underline{\rho}, \bar{\rho}]\right\}$ (i.e., the set of general equilibrium asset holdings) is upper-hemicontinuous and hence $\left\{g_{a}\left(a, r_{\phi}, w\left(r_{\phi}\right), \phi\right): a \in \mathcal{A}, r_{\phi} \in R(\phi) \subset[\underline{\rho}, \bar{\rho}], \phi \in[\underline{\phi}, \bar{\phi}]\right\}$ is a compact set.

We now come back to our initial problem, and to the sequence of steady-state individual allocations $\left(a_{s} \infty\left(\phi_{N}\right), c_{s} \infty\left(\phi_{N}\right), l_{s^{\infty}}\left(\phi_{N}\right), \nu_{s} \infty\left(\phi_{N}\right)\right)_{i}$ corresponding to UI policy $\left(\phi_{N}, \tau_{N}\left(\phi_{N}\right)\right)$, and prices $\left(r\left(\phi_{N}\right), w\left(\phi_{N}\right)\right)$. Because $\left\{g_{a}\left(r_{\phi}, w\left(r_{\phi}\right), \phi\right): r_{\phi} \in R(\phi), \phi \in\right.$ 
$[\underline{\phi}, \bar{\phi}]\}$ is compact, $\left(\phi_{N}, r_{N}\left(\phi_{N}\right),\left(a_{s} \infty\left(\phi_{N}\right)\right)_{s^{\infty} \in \mathcal{S} \infty}\right)_{N \geq 0}$ takes value in a compact set, and we can extract a convergent subsequence $\left(\phi_{N_{k}}, r_{N_{k}}\left(\phi_{N_{k}}\right),\left(a_{s} \infty\left(\phi_{N_{k}}\right)\right)_{s^{\infty} \in \mathcal{S} \infty}\right)_{N_{k} \geq 0}$ with $\phi_{N_{k}} \rightarrow \phi^{*}, r\left(\phi_{N_{k}}\right) \rightarrow r^{*}\left(\phi^{*}\right)$, and $\left(a_{s} \infty\left(\phi_{N_{k}}\right)\right)_{s^{\infty} \in \mathcal{S}^{\infty}} \rightarrow_{a . s .}\left(a_{s^{\infty}}^{*}\left(\phi^{*}\right)\right)_{s^{\infty} \in \mathcal{S}^{\infty}}$.

We deduce by continuity from Lemma 11 that labor supply, consumption, and Lagrange multiplier are also convergent: $\left(c_{s^{\infty}}\left(\phi_{N_{k}}\right), l_{s^{\infty}}\left(\phi_{N_{k}}\right), \nu_{s} \infty\left(\phi_{N_{k}}\right)\right)_{s^{\infty} \in \mathcal{S}^{\infty}} \rightarrow_{a . s}$. $\left(c_{s^{\infty}}^{*}\left(\phi^{*}\right), l_{s^{\infty}}^{*}\left(\phi^{*}\right), \nu_{s^{\infty}}^{*}\left(\phi^{*}\right)\right)_{s^{\infty} \in \mathcal{S}^{\infty} .}$

We conclude the proof of Proposition 5. Define with the notation of Section B.3 similarly to equation (63) for all $N, N_{k}$ :

$$
a_{N, s^{\infty}, N_{k}}=\mathbb{E}\left[a_{s} \infty\left(\phi_{N_{k}}\right) \mid \mathcal{F}_{N}\right]_{s^{\infty}} \text { for all } s^{\infty} \in C_{N}\left(\left\{s^{N}\right\}\right), \text { a.s. }
$$

where $C_{N_{k}}$ is defined in (53). Since we are in a compact set, $\left|a^{i}\left(\phi_{N_{k}}\right)\right|$ is bounded by a constant. We can use the dominated convergence theorem to permute limit (in $N_{k}$ ) and integral in (79). We deduce that:

$$
a_{N, s^{\infty}}=\lim _{N_{k} \rightarrow \infty} a_{N, s^{\infty}, N_{k}}=\mathbb{E}\left[a_{s^{\infty}}^{*}\left(\phi^{*}\right) \mid \mathcal{F}_{N}\right], s^{\infty} \in C_{N}\left(\left\{s^{N}\right\}\right) .
$$

We conclude with a similar argument as in Proposition 2:

$$
\lim _{N} a_{N, s^{\infty}}=a_{s^{\infty}}^{*}\left(\phi^{*}\right), \text { a.s. }
$$

We proceed similarly for consumption, labor supply, and Lagrange multiplier. Finally, it is straightforward to check that the FOCs of the Ramsey aggregate program (Section 4.2) converge to those of the original Ramsey program (Section 3). The FOCs of the original Ramsey program therefore hold for the limit allocation $\left(\phi^{*}, \tau^{*}\left(\phi^{*}\right), r^{*}\left(\phi^{*}\right), w^{*}\left(\phi^{*}\right),\left(c_{s}^{*}\left(\phi^{*}\right)\right.\right.$,

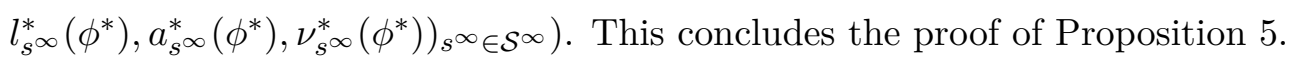

\section{B.4.2 Proof of Proposition 3}

We now further impose the uniqueness of the original Ramsey equilibrium. In other words, the sequence $\left(\phi_{N}, r_{N}\left(\phi_{N}\right),\left(a_{s} \infty\left(\phi_{N}\right)\right)_{s^{\infty} \in \mathcal{S} \infty}\right)_{N \geq 0}$ takes value in a compact set (see Section

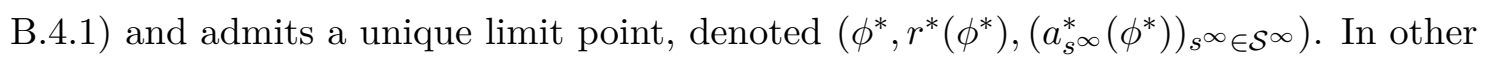
words, there is only one point which can be the limit. Note that again, we only need to look at the triplet $\left(\phi^{*}, r^{*},\left(a_{s^{\infty}}^{*}\left(\phi^{*}\right)\right)_{s^{\infty} \in \mathcal{S}^{\infty}}\right)$, since consumption, wage, labor supply and Lagrange multiplier can be deduced from this triplet. Then, $\left(\phi_{N}, r_{N}\left(\phi_{N}\right),\left(a_{s^{\infty}}\left(\phi_{N}\right)\right)_{s^{\infty} \in \mathcal{S}^{\infty}}\right)_{N \geq 0}$ 
converges to $\left(\phi^{*}, r^{*}\left(\phi^{*}\right),\left(a_{s^{\infty}}^{*}\left(\phi^{*}\right)\right)_{s^{\infty} \in \mathcal{S}^{\infty}}\right)$ (a.s. for assets). Indeed, suppose it is not the case. Then there exists $\varepsilon>0$ n such for all $N \geq 0$, there exists $k \geq N$, such that $\left\|\left(\phi_{k}, r_{k}\left(\phi_{k}\right),\left(a_{s} \infty\left(\phi_{k}\right)\right)_{s^{\infty} \in \mathcal{S}^{\infty}}\right)-\left(\phi^{*}, r^{*}\left(\phi^{*}\right),\left(a_{s^{\infty}}^{*}\left(\phi^{*}\right)\right)_{s^{\infty} \in \mathcal{S}^{\infty}}\right)\right\| \geq \varepsilon$ (the product norm is the Euclidian norm for the two first dimensions and the one associated to the almost sure convergence for the third one). This means that the set:

$$
\begin{aligned}
& \left\{\left(\phi_{N}, r_{N}\left(\phi_{N}\right),\left(a_{s^{\infty}}\left(\phi_{N}\right)\right)_{s^{\infty} \in \mathcal{S}^{\infty}}\right):\right. \\
& \left.\left\|\left(\phi_{N}, r_{N}\left(\phi_{N}\right),\left(a_{s^{\infty}}\left(\phi_{N}\right)\right)_{s^{\infty} \in \mathcal{S}^{\infty}}\right)-\left(\phi^{*}, r^{*}\left(\phi^{*}\right),\left(a_{s^{\infty}}^{*}\left(\phi^{*}\right)\right)_{s^{\infty} \in \mathcal{S}^{\infty}}\right)\right\| \geq \varepsilon\right\}
\end{aligned}
$$

is infinite and compact (as the complement of an open set in a compact set). We can thus extract a convergent subsequence $\left(\phi_{N_{k}}, r_{N_{k}}\left(\phi_{N_{k}}\right),\left(a_{s^{\infty}}\left(\phi_{N_{k}}\right)\right)_{s^{\infty} \in \mathcal{S}^{\infty}}\right)_{n_{k}}$ that converges to a limit $\left(\phi^{* *}, r^{* *},\left(a_{s^{\infty}}^{* *}\left(\phi^{* *}\right)\right)_{s^{\infty} \in \mathcal{S}^{\infty}}\right) \neq\left(\phi^{*}, r^{*},\left(a_{s^{\infty}}^{*}\left(\phi^{*}\right)\right)_{s^{\infty} \in \mathcal{S}^{\infty}}\right)$. This contradicts the uniqueness of the limit point. This completes the proof of Proposition B.3.

\section{Details of the implementation of the truncation method}

This section is organized in three parts. Section C.1 presents all the equations driving the dynamics of the truncated model. Section C.2 details the computations that enable to express the steady-state value of the $\xi$ s using linear algebra.

\section{C.1 Summary of the dynamics of the truncated model}

The dynamics of the model in the presence of an optimal time-varying replacement rate can be written as follows. We start with equations valid for all $s^{N} \in \mathcal{S}^{N}$ :

$$
\begin{aligned}
l_{t, s^{N}} & =\chi^{\varphi}\left(1-\tau_{t}\right)^{\varphi} w_{t}^{\varphi} y_{0}^{N, \varphi} 1_{e_{0}^{N}=e}, \\
c_{t, s^{N}}+a_{t, s^{N}} & \leq\left(1+r_{t}\right) \tilde{a}_{t, s^{N}}+\left(\left(1-\tau_{t}\right) 1_{e_{0}^{N}=e}+\phi_{t} 1_{e_{0}^{N}=u}\right) l_{t, s^{N}} y_{0}^{N} w_{t}, \\
\tilde{a}_{t, s^{N}} & =\sum_{\tilde{s}^{N} \in \mathcal{S}^{N}} \Pi_{t, s^{N} \tilde{s}^{N}} \frac{S_{\tilde{s}^{N}}}{S_{s^{N}}} a_{t-1, \tilde{s}^{N}}, \\
\Psi_{t, s^{N}} & =U_{c, t, s^{N}}-\left(\lambda_{t, s^{N}}-\left(1+r_{t}\right) \tilde{\lambda}_{t, s^{N}}\right) \xi_{s^{N}} U_{c c, t, s^{N}}, \\
\tilde{\lambda}_{t, s^{N}}= & \sum_{\tilde{s}^{N} \in \mathcal{S}^{N}} \frac{S_{t-1, \tilde{s}^{N}}}{S_{t, s^{N}}} \Pi_{t, \tilde{s}^{N} s^{N}} \lambda_{t-1, \tilde{s}^{N}}, \\
S_{t, s^{N}}= & \sum_{\hat{s}^{N} \in \mathcal{S}^{N}} S_{t-1, \hat{s}^{N}} \Pi_{t, \hat{s}^{N} s^{N}} .
\end{aligned}
$$


Then, the equations valid for unconstrained histories only $\left(s^{N} \notin \mathcal{C}\right)$ are:

$$
\begin{aligned}
& \xi_{s^{N}} U_{c, t, s^{N}}=\beta(1+r) \sum_{\tilde{s}^{N} \in \mathcal{S}^{N}} \Pi_{t+1, s^{N} \tilde{s}^{N}} \xi_{\tilde{s}^{N}} U_{c, t+1, \tilde{s}^{N}}, \\
& \Psi_{t, s^{N}}=\beta \sum_{\tilde{s}^{N} \in \mathcal{S}^{N},} \Pi_{t+1, s^{N} \tilde{s}^{N}} \mathbb{E}_{t}\left[\left(1+r_{t+1}\right) \Psi_{t+1, \tilde{s}^{N}}\right]+\beta \frac{1-\alpha}{\varphi} \mathbb{E}_{t}\left[\frac{1}{L_{t+1}}\left(\frac{K_{t}}{L_{t+1}}\right)^{\alpha-1}\right. \\
& \left.\times \sum_{\tilde{s}^{N} \in \mathcal{S}^{N}}\left(\left(1-\tau_{t+1}\right) 1_{\tilde{e}_{0}^{N}=e}+\frac{S_{t+1, e}}{S_{t+1, u}}\left(\tau_{t+1}(1+\varphi)-1\right) 1_{\tilde{e}_{0}^{N}=u}\right) \Psi_{t+1, \tilde{s}^{N}} S_{t+1, \tilde{s}^{N}} l_{t+1, \tilde{s}^{N}} \tilde{y}_{0}^{N}\right],
\end{aligned}
$$

For constrained histories $\left(s^{N} \in \mathcal{C}\right)$, we have $a_{t, s^{N}}+\bar{a}=\lambda_{t, s^{N}}=0$. Aggregate equations are:

$$
\begin{aligned}
& \sum_{s^{N} \in \mathcal{S}^{N}} S_{t, s^{N}} \tilde{\lambda}_{t, s^{N}} \xi_{s^{N}} U_{c, t, s^{N}}=-\sum_{s^{N} \in \mathcal{S}^{N}} S_{t, s^{N}} \Psi_{t, s^{N}} \tilde{a}_{t, s^{N}}+\frac{1}{\alpha \varphi} \frac{K_{t-1}}{L_{t}} \\
& \times \sum_{s^{N} \in \mathcal{S}^{N}}\left(-\left(1-\tau_{t}\right) 1_{e_{0}^{N}=e}+\frac{S_{t, e}}{S_{t, u}}\left(1+\alpha \varphi-(1+\varphi) \tau_{t}\right) 1_{e_{0}^{N}=u}\right) S_{t, s^{N}} \Psi_{t, s^{N}} l_{t, s^{N}} \tilde{y}_{0}^{N}, \\
& K_{t}=\sum_{s^{N} \in \mathcal{S}^{N}} S_{t, s^{N}} a_{t, s^{N}}, L_{t}=\sum_{s^{N} \in \mathcal{S}^{N}} S_{t, s^{N}} y_{0}^{N} l_{t, s^{N}}, \\
& \sum_{s^{N} \in \mathcal{S}^{N}} S_{t, s^{N}} c_{t, s^{N}}+K_{t}=Z_{t} K_{t-1}^{\alpha} L_{t}^{1-\alpha}+(1-\delta) K_{t-1}, \\
& r_{t}=\alpha Z_{t}\left(\frac{K_{t-1}}{L_{t}}\right)^{\alpha-1}-\delta, w_{t}=(1-\alpha) Z_{t}\left(\frac{K_{t-1}}{L_{t}}\right)^{\alpha} \text { and } \log Z_{t}=\rho \log Z_{t-1}+u_{t} .
\end{aligned}
$$

The optimal replacement rate is given by equation (80), corresponding to the first-order condition of the Ramsey program of Section 4.2. Note that the dynamics of the model with an exogenous replacement rate can be deduced from the previous set of equations, in which $\phi$ (and $\tau$ ) has to be set to its exogenous value and equation (80) is discarded.

This system has $Y+5(2 Y)^{N}+7$ equations for the same number of variables. A standard software like Dynare is able to perform automatic differentiation and to identify the set of state variables $\left(\left(a_{t, s^{N}}, \lambda_{t, s^{N}}, S_{t, s^{N}}\right)_{s^{N} \in \mathcal{S}^{N}}, K_{t}, Z_{t}\right)$. It provides the first-order dynamics for the (stacked) vector of level-deviation of state variables (denoted by $y_{t}$ ) from their steady-state values (denoted by $\left.y^{s}\right)$ :

$$
y_{t}-y^{s}=A\left(y_{t-1}-y_{s}\right)+B u_{t},
$$

where $A$ is a square matrix, $B$ a row matrix, and $u_{t}$ is the innovation of the TFP process. See Adjemian et al. (2011) for the details of the implementation of the numerical procedure. 


\section{C.2 Matrix representation of the steady state to compute $\xi$ and $\lambda$}

Before turning to the matrix representation, we introduce the following notation: $\circ$ is the Hadamard product, $\otimes$ the Kronecker product, and $\times$ the usual matrix product. For a vector $V, \operatorname{diag}(V)$ is the diagonal matrix with $V$ on the diagonal.

A truncated history is a vector $s^{N}=\left\{\left(y_{-N+1}, e_{-N+1}\right), \ldots,\left(y_{0}, e_{0}\right)\right\} \in \mathcal{S}^{N}$, where $e_{k}=0$ if the agent is unemployed and $e_{k}=1$ if the agent is employed, and $y_{k}$ is the productivity level, $k$ periods in the past. Productivity levels $(\underline{y}, \ldots, \bar{y})$ are assumed to be ordered and we denote by $i_{y}$ the index function of productivity levels (in increasing order). For instance, $i_{y}(\underline{y})=1$, while $i_{y}(\bar{y})=\operatorname{Card}(\mathcal{Y})$. The total number of histories is $N_{\text {tot }}=S^{N}$ where $S=\operatorname{Card}(\mathcal{S})$.

We can identify each history by the integer $k_{s^{N}}=1, \ldots, N_{t o t}$ defined as:

$$
k_{s^{N}}=\sum_{k=0}^{N-1} N_{t o t}^{-N+1-k}\left(e_{k} \times Y+i_{y}\left(y_{k}\right)-1\right)+1,
$$

which corresponds to an enumeration in base $S$ of the vector $s^{N}$. In this enumeration, the first $N_{\text {tot }} / 2$ histories are histories where agents are currently unemployed, while the other half corresponds to employed histories.

The idea of matrix notation we use below is to stack equations holdings for all $s^{N} \in \mathcal{S}^{N}$ into vectors and matrices, following the order of the enumeration in (81).

\section{C.2.1 Computing the $\xi \mathrm{s}$}

Let $\mathbf{S}$ be the $N_{t o t}$-vector of steady-state history sizes. Similarly, let $\mathbf{a}, \mathbf{c}, \boldsymbol{\ell}, \boldsymbol{\nu}, \mathbf{U}_{\mathbf{c}}, \mathbf{U}_{\mathbf{c c}}$ be the $N_{t o t}$-vectors of end-of-period wealth, consumption, labor supply, Lagrange multipliers, marginal utilities, and derivatives of the marginal utility, respectively. Each vector is computed using equation (23) as the aggregation of the relevant variable - known from the steady-state Bewley model. We also define:

$$
\mathbf{W}=w\left[\begin{array}{c}
\phi \\
1-\tau
\end{array}\right] \otimes \boldsymbol{y} \otimes 1_{B}, \mathbf{L}_{e}=\left[\begin{array}{l}
0 \\
1
\end{array}\right] \otimes \boldsymbol{y} \otimes 1_{B}, \mathbf{L}_{u}=\left[\begin{array}{l}
1 \\
0
\end{array}\right] \otimes \boldsymbol{y} \otimes 1_{B},
$$

where $\boldsymbol{y}=\left[y_{1} \ldots y_{Y}\right]^{\top}$ is the vector of productivity levels. Let $\mathbb{P}$ be the diagonal matrix having 1 on the diagonal at $s^{N}$ if and only if the history $s^{N}$ is not credit constrained (i.e., 
$\left.\nu_{s^{N}}=0\right)$, and 0 otherwise. Similarly, define $\mathbb{P}^{c}=\mathbf{I}-\mathbb{P}$, where $\mathbf{I}$ is the $\left(N_{t o t} \times N_{t o t}\right)$-identity matrix. Let $\Pi$ be the transition matrix across histories. In the steady state:

$$
\begin{gathered}
\mathbf{S}=\Pi \mathbf{S} \text { and } \mathbb{P}^{c} \mathbf{a}=-\bar{a} 1_{N_{t o t} \times 1}, \\
\mathbf{S} \circ \mathbf{c}+\mathbf{S} \circ \mathbf{a}=(1+r) \Pi(\mathbf{S} \circ \mathbf{a})+(\mathbf{S} \circ \mathbf{W} \circ \boldsymbol{\ell}), \\
\left(\frac{r+\delta}{\alpha}\right)^{\frac{1}{\alpha-1}} \mathbf{L}_{\mathbf{e}}^{\top} \times \mathbf{S}=\mathbf{S}^{\top} \times \mathbf{a}, \\
\tau=\phi \frac{\mathbf{L}_{\mathbf{u}}^{\top} \times \mathbf{S}}{\mathbf{L}_{\mathbf{e}}^{\top} \times \mathbf{S}}, \text { and } w=(1-\alpha)\left(\frac{r+\delta}{\alpha}\right)^{\frac{\alpha}{\alpha-1}} .
\end{gathered}
$$

(The previous equalities can be double-checked numerically). We define the vector $\tilde{\xi}$ as:

$$
\tilde{\boldsymbol{\xi}}=\left[\mathbb{P}\left(\operatorname{diag}\left(u^{\prime}(\mathbf{c})\right)-\beta(1+r) \Pi \times \operatorname{diag}\left(u^{\prime}(\mathbf{c})\right)\right)+\mathbb{P}^{c}\right]^{-1} \boldsymbol{\nu},
$$

which is well-defined since the matrix $\mathbb{P}\left(\operatorname{diag}\left(u^{\prime}(\mathbf{c})\right)-\beta(1+r) \Pi \times \operatorname{diag}\left(u^{\prime}(\mathbf{c})\right)\right)+\mathbb{P}^{c}$ is invertible (because $\beta(1+r)<1$, and $\mathbb{P}+\mathbb{P}^{c}=\mathbf{I}$ ), and since the vector $\boldsymbol{\nu}$ is not zero (because some histories are credit constrained due to a credit limit above the natural borrowing limit). With definition (83), we can check that for unconstrained histories, we have:

$$
\tilde{\boldsymbol{\xi}} \circ u^{\prime}(\mathbf{c})=\beta(1+r) \Pi\left(\tilde{\boldsymbol{\xi}} \circ u^{\prime}(\mathbf{c})\right)
$$

and for constrained histories, $\tilde{\boldsymbol{\xi}} \circ u^{\prime}(\mathbf{c})=\beta(1+r) \Pi\left(\tilde{\boldsymbol{\xi}} \circ u^{\prime}(\mathbf{c})\right)+\boldsymbol{\nu}$, where we use $\tilde{\boldsymbol{\xi}} \circ u^{\prime}(\mathbf{c})=$ $\operatorname{diag}\left(u^{\prime}(\mathbf{c})\right) \tilde{\boldsymbol{\xi}}$.

\section{C.2.2 Computing equilibrium Lagrange multipliers $\lambda$}

We derive here the Lagrange multipliers of the Ramsey program as a function of the steady-state solution (i.e., allocations and prices), which is assumed to be known. Denoting the vectors associated with the Lagrange multipliers by $\boldsymbol{\lambda}, \tilde{\boldsymbol{\lambda}}$, and $\boldsymbol{\Psi}$, we have: $\boldsymbol{\Psi}=$ $\boldsymbol{\xi} \circ \mathbf{U}_{\mathbf{c}}-(\boldsymbol{\lambda}-(1+r) \tilde{\boldsymbol{\lambda}}) \circ \boldsymbol{\xi} \circ \mathbf{U}_{\mathbf{c c}}$, from (17). We define the matrix $\Pi^{\Lambda}$ by $\Pi_{\tilde{k} k}^{\Lambda}=\frac{S_{k} \Pi_{k \tilde{k}}}{S_{\tilde{k}}}$, such that $\tilde{\boldsymbol{\lambda}}=\Pi^{\Lambda} \boldsymbol{\lambda}$, and the matrix $\Pi^{\Psi}$ by:

$$
\Pi_{k \tilde{k}}^{\Psi}=\beta\left(1+F_{K}\right) \Pi_{k \tilde{k}}+\beta \frac{1-\alpha}{\alpha \varphi} \frac{r+\delta}{L}\left((1-\tau) 1_{\tilde{k}>\frac{N_{t o t}}{2}}+\frac{S_{e}}{S_{u}}(\tau(1+\varphi)-1) 1_{\tilde{k} \leq \frac{N_{t o t}}{2}}\right) S_{\tilde{k}} l_{\tilde{k}} y_{\tilde{k}},
$$


where $1_{\tilde{k}>\frac{N_{\text {tot }}}{2}}$ represents employed agents and $1_{\tilde{k} \leq \frac{N_{\text {tot }}}{2}}$ unemployed ones. It can be checked from (18) that $\mathbb{P} \boldsymbol{\Psi}=\mathbb{P} \Pi^{\Psi} \boldsymbol{\Psi}$ and that the vector of the Lagrange multipliers, $\boldsymbol{\lambda}$, verify:

$$
\boldsymbol{\lambda}=\left[\mathbb{P}^{c}+\mathbb{P}\left(\mathbf{I}-\Pi^{\Psi}\right)\left(\operatorname{diag}\left(\boldsymbol{\xi} \circ \mathbf{U}_{\mathbf{c c}}\right)\left(\mathbf{I}-\left(1+F_{K}\right) \Pi^{\Lambda}\right)\right)\right]^{-1} \mathbb{P}\left(\mathbf{I}-\Pi^{\Psi}\right)\left(\boldsymbol{\xi} \circ \mathbf{U}_{\mathbf{c}}\right) .
$$

Importantly, the right-hand side can be deduced from the Bewley allocations, which makes the computation of $\boldsymbol{\lambda}$ straightforward. We then deduce $(\tilde{\boldsymbol{\lambda}}, \Psi)$ with:

$$
\tilde{\boldsymbol{\lambda}}=\Pi^{\Lambda} \lambda, \text { and } \boldsymbol{\Psi}=\xi \circ \mathbf{U}_{c}-\xi \circ \mathbf{U}_{\mathbf{c c}} \circ\left(\mathbf{I}-\left(1+F_{K}\right) \Pi^{\Lambda}\right) \lambda .
$$

Equation (34) that allows to check for the optimality of the planner's instruments is:

$$
\mathbf{V}^{\top} \times 1_{N_{t o t} \times 1}=0
$$

where: $\mathbf{V} \equiv \frac{\varphi \alpha}{K}\left(\mathbf{S} \circ \Psi \circ \tilde{\mathbf{a}}+\mathbf{S} \circ \Lambda \circ \xi \circ \mathbf{U}_{c}\right)+\frac{1-\tau}{L} \mathbf{S} \circ \Psi \circ \mathbf{L}^{e}-(1-\tau+\varphi(\alpha-\tau)) \frac{S_{e}}{S_{u}} \mathbf{S} \circ \Psi \circ \mathbf{L}^{u}$.

\section{Supplemental numerical results}

\section{D.1 Computational methods}

For all simulation methods, we first solve for the steady-state allocation of the original Bewley model. The consumer's problem is solved on a grid using the Endogenous Grid Point Method (EGM) (Carrol, 2006 or Den Haan, 2010). For the decision rules, the asset grid has 50 points, non-linearly spaced, as in Boppart et al. (2018), and households can choose points off the grid by linear interpolation. There are 7 different productivity levels and 2 employment status. We thus solve for 14 policy rules. The Euler equation errors reported in Table 7 show that the accuracy is satisfactory.

Truncated model. To derive the truncated model for a given $N$, we first use the steadystate distribution of the original Bewley model to compute average consumption and savings in each of the 14 idiosyncratic states. We then use the policy rules $g_{i}(a)$ for savings and $g_{i}^{c}(a)$ for consumption, together with the transition probabilities, $\left(\Pi_{i, j}\right)_{i, j=1, \ldots, 14}$, to compute the average consumption and saving levels for each idiosyncratic history. For example, when we know the steady-state beginning-of-period distribution of wealth, $\Lambda_{i}(a)$, of agents in states $i=1, \ldots, 14$, we can compute the steady-state distribution of wealth of 
agents with history $(i, j), \Lambda_{(i, j)}(a)$ by computing:

$$
\Lambda_{(i, j)}\left(a^{\prime}\right)=\Pi_{i, j} \int_{a, g_{i}(a)=a^{\prime}} \Lambda_{i}(a) d a .
$$

Once we have the distributions $\Lambda_{(i, j)}$, the average consumption levels and savings by history are simply constructed by $c_{i, j}=\int_{a} g_{j}^{c}(a) \Lambda_{(i, j)}(a) d a$. and $a_{i, j}=\int_{a} a \Lambda_{(i, j)}(a) d a$ (note that we use the policy rule of the last states). For histories $(i, j, k)$ with $i, j, k=1, \ldots, 14$, we start from $\Lambda_{(i, j)}$ and construct $\Lambda_{(i, j, k)}$ as in (87). This strategy allows us to construct recursively the steady-state distribution of wealth for any idiosyncratic history of arbitrary length. Once the average values are computed, we deduce the $\xi s$ using equation (83). Note that the Dynare solver can be used to double-check that the steady state computed in Section C.2 is indeed a steady state of the dynamic equations. Finally, we then compute a first-order approximation for the aggregate shock of the whole system of equations, given in Section C.1, using Dynare again.

Reiter model. We implement the algorithm described in Reiter (2009), which is now standard. For each asset level and for each idiosyncratic state, we perform a first-order approximation of the policy rule for the aggregate states. We use these approximated policy rules to simulate the dynamics of the model for 10,000 periods.

Boppart, Krusell, and Mitman. Following Boppart et al. (2018), we first simulate an unexpected shock (MIT shock) to the innovation $\varepsilon_{t}^{z}$, to compute the IRFs for the various variables of interest. The transition path is then solved by iteration on the capital path, assuming that the economy comes back to its steady state after 400 periods. These IRFs are then used as numerical partial derivatives for any variable $x_{t}$ under consideration according to the aggregate shock (which is continuous), at different time-horizons, namely $\frac{\partial x_{t+k}}{\partial \varepsilon_{t}^{z}}$ for $k=0, \ldots, T$ (where $T$ is chosen high enough for the derivative to be negligible for $k>T)$. These derivatives are then used to simulate the economy with aggregate shocks, using a Taylor-expansion: $x_{t}=\bar{x}^{s s}+\sum_{k=0}^{T} \frac{\partial x_{t}}{\partial \varepsilon_{t-k}^{z}} \varepsilon_{t-k}^{z}$ for the simulated history associated to the innovation $\varepsilon_{t}^{z}$. The results of these comparisons are presented in Section 6.1. 


\section{D.2 Comparison with other solution methods}

Correlation tables We report in Table 8 the correlations between key-variables for the Reiter's and the truncation method (Economies (3) and (4)) of Section 6.1. The variables are: aggregate consumption $C$, GDP $Y$, capital $K$, TFP $Z$, and aggregate labor supply $L$. Again, one can check that the results are very similar to each other.

\begin{tabular}{cccccc}
\hline & $C$ & $Y$ & $K$ & $Z$ & $L$ \\
\hline$C$ & 1.00 & 0.96 & 0.95 & 0.80 & 0.96 \\
$Y$ & 0.96 & 1.00 & 0.83 & 0.93 & 1.00 \\
$K$ & 0.95 & 0.83 & 1.00 & 0.57 & 0.83 \\
$Z$ & 0.80 & 0.93 & 0.57 & 1.00 & 0.93 \\
$L$ & 0.96 & 1.00 & 0.83 & 0.93 & 1.00 \\
\hline
\end{tabular}

Truncation Method

\begin{tabular}{cccccc}
\hline & $C$ & $Y$ & $K$ & $Z$ & $L$ \\
\hline$C$ & 1.00 & 0.96 & 0.94 & 0.80 & 0.96 \\
$Y$ & 0.96 & 1.00 & 0.81 & 0.94 & 1.00 \\
$K$ & 0.94 & 0.81 & 1.00 & 0.55 & 0.81 \\
$Z$ & 0.80 & 0.94 & 0.55 & 1.00 & 0.94 \\
$L$ & 0.96 & 1.00 & 0.81 & 0.94 & 1.00 \\
\hline \multicolumn{5}{c}{ Reiter method }
\end{tabular}

Table 8: Correlation tables

Comparison for a lower persistence of idiosyncratic shocks In the benchmark economy, the persistence of the idiosyncratic risk is high, consistently with the data (see Table 1). One may want to check that the accuracy of the truncation method does not depend on this high persistence. To do so, we simulate the same economy with the same parameters, except that we set the persistence of the idiosyncratic risk to $\rho_{y}=0.96$ (instead of $\left.\rho_{y}=0.992\right)$. Results are reported in Economies (5)-(8) of Table 9. Once again, it can be checked that the three solution methods generate very similar results, and that the $\xi s$ are useful in reproducing the dynamics in the truncated economy.

\section{E Supplemental theoretical results}

This section contains three parts. Section E.1 provides a simple example to illustrate why the sign of the Lagrange multiplier on the Euler equation of agent $i$ can be interpreted as the perception by the planner of the quantity of savings of agent $i$. Section E.2 presents an economy in which credit constraints have been substituted by penalty functions. Finally, Section E.3 generalizes the truncation theory to non-GHH utility functions. 


\begin{tabular}{llrrrr}
\hline Methods & & BKM & Reiter & Trunc. & $\begin{array}{c}\text { Trunc. } \\
(\xi=1)\end{array}$ \\
\hline Simulations & & $(1)$ & $(2)$ & \multicolumn{1}{c}{$(3)$} & $(4)$ \\
\hline$Y$ & mean & 1.11 & 1.11 & 1.11 & 1.11 \\
& std/mean (\%) & 1.77 & 1.77 & 1.76 & 1.78 \\
$C$ & mean & 0.82 & 0.82 & 0.82 & 0.82 \\
& std/mean (\%) & 1.47 & 1.48 & 1.47 & 1.48 \\
$L$ & mean & 0.29 & 0.29 & 0.29 & 0.29 \\
& std/mean (\%) & 0.59 & 0.59 & 0.59 & 0.59 \\
$K$ & mean & 11.77 & 11.77 & 11.77 & 11.77 \\
& std/mean (\%) & 1.64 & 1.66 & 1.59 & 1.65 \\
$\operatorname{corr}\left(C, C_{-1}\right)$ & $($ in \%) & 99.15 & 99.15 & 99.10 & 99.17 \\
$\operatorname{corr}\left(Y, Y_{-1}\right)$ & & 97.52 & 97.53 & 97.47 & 97.54 \\
$\operatorname{corr}(C, Y)$ & & 96.28 & 96.25 & 96.33 & 99.06 \\
\hline
\end{tabular}

Table 9: Moments of the simulated model for different computational techniques and a low persistence.

\section{E.1 Understanding Lagrange multipliers on Euler equations}

The analysis of the main text uses Lagrange multipliers on Euler equations and claims that these multipliers can be either positive or negative and that their sign is related to the distortions on the saving incentives (from the planner's point of view). This section provides a very simple example (textbook style) to illustrate this statement. In addition, it clarifies some properties of exterior penalty functions, which are used in Section E.2 below.

Consider an economy where the planner has an instrument $\tau$, and the agent can choose a variable $a$. The agent maximizes a concave objective with a constraint $a \geq-\bar{a}$ :

$$
\max _{a \in \mathbb{R}}-(a-\tau)^{2}-\tau^{2}, \quad \text { s.t. } a \geq-\bar{a},
$$

which yields the FOC ( $\nu$ being the credit-constraint Lagrange multiplier):

$$
a-\tau=\nu
$$

This corresponds to $a=\tau$ if $\tau \geq-\bar{a}$ or $a=-\bar{a}$ if $\tau<-\bar{a}$. 
The planner's program can now be written as:

$$
\begin{aligned}
& \max _{a, \tau \in \mathbb{R}}-(a-\tau)^{2}-\tau^{2} \\
& \text { s.t. } a-\tau=\nu \text { and } a \geq-\bar{a} .
\end{aligned}
$$

The Lagrangian is ( $\lambda$ is the Lagrange multiplier on the agent's FOC and $\mu$ the multiplier on the credit-constraint): $\mathcal{L}=-(a-\tau)^{2}-\tau^{2}-2 \lambda(a-\tau)-2 \mu(a+\bar{a})$, which yields:

$$
\begin{array}{r}
a-\tau+\lambda+\mu=0 \\
-a+2 \tau-\lambda=0
\end{array}
$$

Note that we have $\lambda \mu=0$ and $\lambda \nu=0$. There are two possible cases, depending on whether the constraint $a \geq-\bar{a}$ is binding or not (i.e., whether $\lambda \neq 0$ or not):

1. $\lambda=0$. So, we have $\mu=\nu=0$. Equation (88) implies $a=\tau$ and equations (89) and (90) become: $\lambda=0$ and $\tau-\lambda=0$. So the solution is:

$$
\lambda=\nu=\mu=0, \text { and } a=\tau=0 .
$$

2. $\lambda \neq 0$. So $a=-\bar{a}$ and equations (89) and (90) become:

$$
-\mu=\nu=-\frac{\bar{a}}{2}, \text { and } \tau=-\frac{\bar{a}}{2} .
$$

\section{Penalty functions}

We define the penalty function $g$ as follows:

$$
g(a)=\frac{1}{2} \max (-\bar{a}-a, 0)^{2}, \quad g^{\prime}(a)=-\max (-\bar{a}-a, 0), \quad g^{\prime \prime}(a)=1_{a \leq-\bar{a}} .
$$

Agent's program. Let $\gamma$ be the weight of the penalty function, the agent's program is: $\max _{a \in \mathbb{R}}-(a-\tau)^{2}-\tau^{2}-2 \gamma g(a)$. The FOC yields:

$$
a-\tau-\gamma \max (-\bar{a}-a, 0)=0
$$

So, there are two solutions: (i) $a=\tau$ if $-\bar{a}-\tau \leq 0$, or $a=\frac{\tau}{1+\gamma}+\frac{\gamma}{1+\gamma}(-\bar{a})$ if $-\bar{a}-\tau>0$. We can observe than when $\gamma \rightarrow \infty$, these two solutions converge to the solutions (91) and (92) of the Ramsey problem. 
Planner's program. For the sake of generality, we consider a penalty function with a different coefficient $\tilde{\gamma}=\kappa \gamma$, where we can have $0<\kappa \leq 1$ or $\kappa>1$, depending on who (the agent of the planner) gives the constraint the highest value. The planner's program is: $\max _{a, \tau \in \mathbb{R}}-(a-\tau)^{2}-\tau^{2}-2 \tilde{\gamma} g(a)$, s.t. $a-\tau=-\gamma g^{\prime}(a)$. The Lagrangian is: $\mathcal{L}=-(a-\tau)^{2}-\tau^{2}-2 \lambda\left(a-\tau+\gamma g^{\prime}(a)\right)+2 \tilde{\gamma} g(a)$, with the FOCs:

$$
\begin{aligned}
0 & =\frac{a+\lambda}{2}+\lambda \gamma 1_{a \leq-\bar{a}}-\tilde{\gamma} \max (-\bar{a}-a, 0), \\
\tau & =\frac{a+\lambda}{2}
\end{aligned}
$$

Again, there are two cases:

1. $a>-\bar{a}$. Then (93), (94) and (95) imply: $a=\tau=\frac{a+\lambda}{2}=0$.

2. $a \leq-\bar{a}$. Then (93) implies $\tau=(\gamma+1) a+\gamma \bar{a}$. We obtain using (94) and (95):

$$
a=\frac{2 \gamma^{2}+(\kappa+1) \gamma}{2 \gamma^{2}+(\kappa+2) \gamma+1} \bar{a}, \tau=-\frac{\gamma^{2}+\kappa \gamma}{2 \gamma^{2}+(\kappa+2) \gamma+1} \bar{a}, \lambda=-\frac{(\kappa-1) \gamma}{2 \gamma^{2}+(\kappa+2) \gamma+1} \bar{a} .
$$

Remark 1 (Sign of $\lambda$ ) The sign of $\lambda$ can be positive or negative, depending on whether $\kappa>1$ or $\kappa<1$, which indicates who (between the planner and the agent) gives the credit constraint the highest value.

At the limit: $a \rightarrow-\bar{a}, \lambda \rightarrow 0, \tau \rightarrow-\frac{\bar{a}}{2}$, which is the solution of the initial program.

\section{E.2 Penalty functions}

In this section, we replace the credit constraint with a penalty function denoted by $g(a)$ for a saving choice $a$. The functional form of $g$ is standard for exterior penalty function (see Luenberger and Ye, 2016 for a textbook treatment of penalty functions). For a saving $a<-\bar{a}$, the distance to the credit limit is $-\bar{a}-a$. The baseline penalty function is thus $g(a)=\max (-\bar{a}-\underline{a}, 0)^{2}$. We parametrize this penalty function by a scalar $\gamma>0$ such that all agents face a penalty function $\gamma g(a)$. We denote with a $\gamma$ superscript the quantities associated to penalty function $\gamma g(a)$. The goal of this section is to show that the FOCs (39) and (40) are limits of FOCs for infinitely concave penalty functions, i.e., for $\gamma \rightarrow \infty$. 


\section{E.2.1 The truncated economy}

In the presence of the penalty function, the agent's program can be written as

$$
\begin{gathered}
\left.\max _{\left(c_{t}^{\gamma, i}, l_{t}^{\gamma, i}, a_{t}^{\gamma, i}\right)_{t \geq 0, i}} \tilde{J}_{i}=\mathbb{E}_{0} \sum_{t=0}^{\infty} \beta^{t}\left(\xi_{s^{N}} U\left(c_{t}^{\gamma, i}, \hat{l}_{t}^{\gamma, i}\right)-\gamma g\left(a_{t}^{\gamma, i}\right)\right)\right], \\
a_{t}^{\gamma, i}+c_{t}^{\gamma, i}=\left(\left(1-\tau_{t}\right) l_{t}^{\gamma, i} 1_{e_{0}^{N}=e}+\phi_{t} l_{t, s^{N}, e^{\gamma}}^{\gamma} e_{0}^{N}=u\right. \\
c_{t}^{\gamma, i}, l_{t}^{\gamma, i} \geq 0 .
\end{gathered}
$$

with given initial conditions. Compared to the initial program (5)-(7), the objective function (96) includes penalty functions, while credit constraint have been removed.

As the replacement rate is exogenous, we make the following additional assumption.

Assumption C We assume that for all $t \geq 0: 1-\tau_{t}>\frac{\varphi}{1+\varphi}$ and $\phi_{t}>\frac{\varphi}{1+\varphi}$.

Assumption C is purely technical: it guarantees that the utility - with a GHH utility function - of employed and unemployed agents in autarky is well-defined and finite. This assumption is obviously verified in our numerical exercise of Section 5.

Lemma 12 For any $\gamma>0$, we have $\tilde{J}_{\gamma}>-\infty$, where $\tilde{J}_{\gamma}$ is defined in equation (96).

Lemma 12 states that the agent's welfare in the economy is well-defined and finite for any values of $\gamma$. It is a direct consequence of Assumption C.

Proof. We prove that the autarky allocation (i.e., null savings at all dates) is feasible. We assume that $a_{t}^{a u t, i}=0$, for all $t \geq 0$ and all $i$. The consumption of an agent when employed and with productivity $y$ is $c_{t}^{a u t, i}=(1-\tau) l_{t}^{a u t, i}(y) y w_{t}$. Since her labor supply is $l_{t}^{a u t, i}(y)=\left(\chi w_{t} y\right)^{\varphi}$, we have:

$$
c_{t}^{a u t, i}-\frac{1}{\chi} \frac{l_{t}^{a u t, i}(y)^{1+\frac{1}{\varphi}}}{1+\frac{1}{\varphi}}=\left(1-\tau-\frac{\varphi}{1+\varphi}\right) \frac{\left(\chi w_{t} y\right)^{1+\varphi}}{\chi},
$$

Similarly for the unemployment case, from which $\zeta(y)=l(y)$ :

$$
c_{t}^{a u t, i}-\frac{1}{\chi} \frac{l_{t}^{a u t, i}(y)^{1+\frac{1}{\varphi}}}{1+\frac{1}{\varphi}}=\left(\phi-\frac{\varphi}{1+\varphi}\right) \frac{\left(\chi w_{t} y\right)^{1+\varphi}}{\chi},
$$

Assumption C ensures that quantities (99) and (100) are bounded away from zero for all $\gamma$. The autarky allocation is thus feasible (independently of $\gamma$ ), and has a finite welfare, which concludes the proof. 
We now derive the FOCs in the truncated economy and investigate their convergence when $\gamma \rightarrow \infty$. Following the same steps as in Section 4, we deduce from the program (96) the following Euler equations:

$$
\begin{aligned}
l_{t, s^{N}}^{\gamma} & =\chi\left(1-\tau_{t}\right) y_{0}^{N} w_{t} U_{c, t, s^{N}}^{\gamma}, \\
\xi_{s^{N}} U_{c, t, s^{N}}^{\gamma} & =\gamma g^{\prime}\left(a_{t, s^{N}}^{\gamma}\right)+\beta \mathbb{E}_{t}\left[\sum_{\tilde{s}^{N}} \Pi_{t+1, s^{N}, \tilde{s}^{N}}\left(1+r_{t+1}\right) \xi_{\tilde{s}^{N}} U_{c, t+1, s^{N}}^{\gamma}\right],
\end{aligned}
$$

with $U_{c, t, s^{N}}^{\gamma}=U_{c, t, s^{N}, \gamma}\left(c_{t, s^{N}}^{\gamma}, l_{t, s^{N}}^{\gamma}\right)$. Compared to Euler equations (39) and (40), the labor Euler equation (101) remains unchanged, while the consumption Euler equation (102) does not feature a Lagrange multiplier for the credit constraint but instead the derivative of the penalty function. All other equations characterizing the truncated equilibrium are unchanged (factor prices, market clearing conditions, etc.).

We can now state our result regarding the limit Euler equation.

Lemma 13 (Limit penalty) When $\gamma \rightarrow \infty$, the solution of (36) is such that we have: $\lim _{\gamma \rightarrow \infty} a_{t, s^{N}}^{\gamma}=-\bar{a}$ or, defining $c_{t, s^{N}}^{\infty}=\lim _{\gamma \rightarrow \infty} c_{t, s^{N}}^{\gamma}$ and $\hat{l}_{t, s^{N}}^{\infty}=\lim _{\gamma \rightarrow \infty} \hat{l}_{t, s^{N}}^{\gamma}$ :

$$
\xi_{s^{N}} U_{c}\left(c_{t, s^{N}}^{\infty}, \hat{l}_{t, s^{N}}^{\infty}\right)=\beta \mathbb{E}_{t}\left[\sum_{\tilde{s}^{N}} \Pi_{t+1, s^{N}, \tilde{s}^{N}}\left(1+r_{t+1}\right) \xi_{\tilde{s}^{N}} U_{c}\left(c_{t+1, s^{N}}^{\infty}, \hat{l}_{t+1, s^{N}}^{\infty}\right)\right] .
$$

Lemma 13 states that, when the penalty function becomes infinitely concave, then either the borrowing of agents facing a positive penalty tends toward the borrowing limit, or their limit allocation verifies Euler equation (103), which is the same as Euler equation (8) in the baseline truncated economy.

Proof. The proof is performed by contradiction. Assume that there exists $s^{N} \in \mathcal{S}^{N}$, such that $\lim _{\gamma \rightarrow \infty} a_{t, s^{N}}<-\bar{a}$. Then, there exists $\varepsilon>0$, such that $a_{t, s^{N}} \leq-\bar{a}-\varepsilon$, for $\gamma$ high enough, which implies that $\lim _{\gamma \rightarrow \infty} \gamma g\left(a_{t, s^{N}}\right)=\lim _{\gamma \rightarrow \infty} \gamma \varepsilon^{2}=\infty$. Hence, $\lim _{\gamma \rightarrow \infty} \tilde{J}_{\gamma}=-\infty$, which contradicts Lemma 12. The second part stems from (102), as $\gamma g^{\prime}\left(a_{t, s^{N}}^{\gamma}\right)=0$ if $a_{t, s^{N}}^{\gamma}>-\bar{a}$. 


\section{E.2.2 The Ramsey program}

We now rewrite the Ramsey program in the presence of penalty functions. The planner's program can be written as follows - we drop the dependence in $\gamma$ to lighten the notation: ${ }^{25}$

$$
\begin{aligned}
& \max _{\left(\left(a_{t, s^{N}}, c_{t, s^{N}}, l_{t, s^{N}}\right)_{s^{N}}, \phi_{t}, \tau_{t}\right)} \mathbb{E}_{t \geq 0}\left[\sum_{t=0}^{\infty} \beta^{t} \sum_{s^{N} \in \mathcal{S}^{N}} S_{t, s^{N}}\left(\xi_{s^{N}} U\left(c_{t, s^{N}}, \hat{l}_{t, s^{N}}\right)-\gamma g\left(a_{t, s^{N}}\right)\right)\right], \\
& \text { s.t. } \xi_{s^{N}} U_{c, t, s^{N}}=\gamma g^{\prime}\left(a_{t, s^{N}}\right)+\beta \mathbb{E}_{t}\left[\left(1+r_{t+1}\right) \sum_{\tilde{s}^{N} \in \mathcal{S}^{N}} \Pi_{t+1, s^{N} \tilde{s}^{N}} \xi_{\tilde{s}^{N}} U_{c, t+1, \tilde{s}^{N}}\right]
\end{aligned}
$$

and subject to the same equations as in the main text: (25), (29), (14), and (3). There are only two differences compared with the Ramsey program in the main text: (i) the presence of penalty functions in the planner's objective; and (ii) penalty functions in the Euler equations (105). We can now state our main equivalence result.

Proposition 6 (Equivalence result) The solution of program (30) is a solution of the program (104) when the penalty function become infinitely concave.

\section{E.2.3 Proof of Proposition 6}

Using Lemma 13, it only remains to be proven that the FOCs of the Ramsey program (104) converge to those of the Ramsey program (30) when penalty costs become infinitely large.

Rewriting the Ramsey program. Denoting by $\beta^{t} S_{t, s^{N}} \lambda_{t, s^{N}}$ the Lagrange multiplier on (105) and using (32), the planner's objective, denoted by $J_{\gamma}$, can be expressed as:

$$
\begin{aligned}
J_{\gamma} & =\mathbb{E}_{0} \sum_{t, s^{N}} \beta^{t} S_{t, s^{N}} \xi_{s^{N}}\left(U_{t, s^{N}}-\left(\lambda_{t, s^{N}}-\left(1+r_{t}\right) \tilde{\lambda}_{t, s^{N}}\right) U_{c, t, s^{N}}\right), \\
& +\mathbb{E}_{0} \sum_{t, s^{N}} \beta^{t} S_{t, s^{N}}\left(-\gamma g\left(a_{t, s^{N}}\right)+\lambda_{t, s^{N}} \gamma g^{\prime}\left(a_{t, s^{N}}\right)\right) .
\end{aligned}
$$

Solving the Ramsey program. The Ramsey program then consists in maximizing $J_{\gamma}$ in (106) over $\left(\left(a_{t, s^{N}}, c_{t, s^{N}}, l_{t, s^{N}}\right)_{s^{N}}, \phi_{t}, \tau_{t}\right)_{t \geq 0}$ subject to the relevant constraints. As in the baseline case, this problem can thus be seen as depending only on saving choices $\left(a_{t, s^{N}}\right)$ and the replacement rate $\phi_{t}$. The FOC for $\phi_{t}$ is independent of penalty functions and is

\footnotetext{
${ }^{25}$ As illustrated in Section E.1, we can choose the same penalty $\gamma$ for the agents and the planner.
} 
identical to (19). We only focus here on the FOC with respect to $\left(a_{t, s^{N}}\right)$, which is after some algebraic manipulations:

$$
\begin{aligned}
\frac{\Psi_{t, s^{N}}}{\beta}=\sum_{\tilde{s}^{N}} \mathbb{E}_{t}\left[\Psi _ { t , \tilde { s } ^ { N } } \left(\left(1+r_{t+1}\right) \Pi_{t+1, s^{N} \tilde{s}^{N}}+S_{t+1, \tilde{s}^{N}}\left(F_{K K, t+1}+\frac{\varphi L_{t+1} \frac{F_{K L, t+1}^{2}}{F_{L, t+1}}}{1-\varphi L_{t+1} \frac{F_{L L, t+1}}{F_{L, t+1}}}\right) \tilde{a}_{t, s^{N}}\right.\right. \\
\left.\left.\quad+(1+\varphi) S_{t+1, \tilde{s}^{N}} \tilde{\tau}_{t+1} l_{\tilde{s}^{N}, e, t+1} \tilde{y}_{0}^{N} \frac{F_{K L, t+1}}{1-\varphi L_{t+1} \frac{F_{L L, t+1}}{F_{L, t+1}}}\right)\right] \\
+\mathbb{E}_{t} \sum_{\tilde{s}^{N}} S_{t+1, \tilde{s}^{N}}\left(F_{K K, t+1}+\frac{\varphi L_{t+1} \frac{F_{K L, t+1}^{2}}{F_{L, t+1}}}{1-\varphi L_{t+1} \frac{F_{L L, t+1}}{F_{L, t+1}}}\right) \tilde{\lambda}_{t+1, \tilde{s}^{N}} \xi_{\tilde{s}^{N}} U_{c, \tilde{s}^{N}, t+1} \\
-\gamma g^{\prime}\left(a_{t, s^{N}}\right)+\lambda_{t, s^{N}} \gamma g^{\prime \prime}\left(a_{t, s^{N}}\right) .
\end{aligned}
$$

Due to the exterior penalty function, for histories for which $a_{t, s^{N}} \leq-\bar{a}, a_{t, s^{N}} \rightarrow-\bar{a}$ as $\gamma \rightarrow \infty$ (Assumption $\mathrm{C}$ has to be fulfilled at any optimal equilibrium). In addition, the previous equality implies that $\lambda_{t, s^{N}} \rightarrow 0$ when $\gamma \rightarrow \infty$ and that the constraints "disappear", as was shown in Section E.1. See Luenberger and Ye (2016) for a proof in a more general case. Finally, for histories for which $a_{t, s^{N}}>\bar{a}$, the previous constraint converges to the same constraint as in the initial truncated program (given in (33)). In our truncated equilibrium without penalty function, we have $\lambda_{t, s^{N}}=0$ and $a_{t, s^{N}}=-\bar{a}$ for the credit-constrained history. As the FOC for $\phi_{t}$ is the same in our initial problem and in the problem with a penalty function, our allocation is therefore a solution of the limit of program with infinitely concave penalty functions. This concludes the proof.

\section{E.3 Generalizing the truncation theory to non-GHH utility functions}

We generalize the truncation method to a separable instantaneous utility function, $U(c, l)=$ $u(c)-v(l)$, instead of the GHH utility function of the main text. The functions $u$ and $v$ are supposed to be continuous, twice differentiable, increasing, and concave in both arguments. For the sake of clarity, the presentation follows the same structure as in the main text.

\section{E.3.1 The set-up}

Besides this more general utility function, the rest of the economy is strictly similar to the economy presented in Section 2. 
The agent's program can now be written as:

$$
\begin{aligned}
\max _{\left\{c_{t}^{i}, l_{t}^{i}, a_{t}^{i}\right\}_{t=0}^{\infty}} & \mathbb{E}_{0} \sum_{t=0}^{\infty} \beta^{t}\left(u\left(c_{t}^{i}\right)-v\left(l_{t}^{i} 1_{e_{t}^{i}=e}+\zeta_{y_{t}^{i}} 1_{e_{t}^{i}=u}\right)\right), \\
c_{t}^{i}+a_{t}^{i} & =\left(1+r_{t}\right) a_{t-1}^{i}+\left(\left(1-\tau_{t}\right) l_{t}^{i} 1_{e_{t}^{i}=e}+\phi_{t} \bar{l}_{t}\left(y_{t}^{i}\right) 1_{e_{t}^{i}=u}\right) y_{t}^{i} w_{t}, \\
& a_{t}^{i} \geq-\bar{a}, c_{t}^{i}>0, l_{t}^{i}>0 .
\end{aligned}
$$

Compared to (5)-(7), the objective function reflects the different utility function, but the constraints are unchanged. The agents' first-order conditions become:

$$
\begin{aligned}
u^{\prime}\left(c_{t}^{i}\right) & =\beta \mathbb{E}_{t}\left[\left(1+r_{t+1}\right) u^{\prime}\left(c_{t+1}^{i}\right)\right]+\nu_{t}^{i}, \\
v^{\prime}\left(l_{t}^{i}\right) & =\left(1-\tau_{t}\right) w_{t} y_{t}^{i} u^{\prime}\left(c_{t}^{i}\right),
\end{aligned}
$$

where the Euler equation (111) obviously only holds for employed agents.

Market clearing conditions (12) are unchanged, and the only difference is that Euler equations for consumption and labor are more involved than in the GHH case. In particular, the individual labor supply is no longer a linear function of productivity.

\section{E.3.2 The truncated model}

Aggregation. As in the GHH economy, we aggregate individual allocations along the truncated history of agents (i.e., their individual idiosyncratic history over the last $N$ periods, $N$ being a given truncation length). The process is very similar (for the budget constraint, and market clearing conditions for instance) and the only difference concerns Euler equations. The aggregation of (112) and (113) yields:

$$
\begin{aligned}
& \xi_{s^{N}, t} u^{\prime}\left(c_{t, s^{N}}\right)=\beta \mathbb{E}_{t}\left[\sum_{\tilde{s}^{N} \succeq s^{N}} \Pi_{t+1, s^{N}, \tilde{s}^{N}} \xi_{\tilde{s}^{N}, t} u^{\prime}\left(c_{t+1, \tilde{s}^{N}}\right)\left(1+r_{t+1}\right)\right]+\nu_{t, s^{N}}, \\
& \xi_{s^{N}, t}^{l} v^{\prime}\left(l_{t, s^{N}}\right)=\left(1-\tau_{t}\right) w_{t} y_{0}^{N} \xi_{s^{N}, t} u^{\prime}\left(c_{t, s^{N}}\right),
\end{aligned}
$$

where $\left(\xi_{s^{N}, t}\right)_{s^{N}}$ have the same interpretation as in the GHH economy and $\left(\xi_{s^{N}, t}^{l}\right)_{s^{N}}$ are their counterparts that are due to the non-linearity of the Euler equation (113) for labor (unlike in the GHH economy). 
Steady-state economy. Prices and allocations at the steady state of the aggregated economy are characterized by the Euler equations for consumption and labor (112) and (113), the collection of (unchanged) budget constraints (25), and the (unchanged) dynamics of history sizes (22). We have the following for the Euler equations:

$$
\begin{aligned}
& \xi_{s^{N}} u^{\prime}\left(c_{s^{N}}\right)=\nu_{s^{N}}+\beta(1+r) \sum_{\tilde{s}^{N} \succeq s^{N}} \Pi_{s^{N}, \tilde{s}^{N}} \xi_{\tilde{s}^{N}} u^{\prime}\left(c_{\tilde{s}^{N}}\right), \\
& \xi_{s^{N}}^{l} v^{\prime}\left(l_{s^{N}}\right)=\left(1-\tau_{t}\right) w_{t} y_{0}^{N} \xi_{s^{N}} u^{\prime}\left(c_{s^{N}}\right),
\end{aligned}
$$

As in the GHH economy, the steady-state equilibrium is further characterized by some unchanged equations: market clearing equations (29), UI scheme budget balance (14), and factor prices (3).

Computing the $\xi$ s and $\xi^{l} \mathbf{s}$. Determining the $\xi_{\mathrm{s}}$ and $\xi^{l} \mathrm{~s}$ follows exactly the same logic as in the GHH economy, using the Bewley allocations, in particular of consumption and labor. The $\xi \mathrm{s}$ (for consumption) are determined such that the aggregated consumption levels (for each history) verify the steady-state consumption Euler equation (114). The difference compared with the GHH case is that, due to the separability of the instantaneous utility function in consumption and labor, this operation only requires consumption allocations of the Bewley model. However, this separability also enables us to determine the $\xi^{l}$ s. This computation is straightforward and for each history, the $\xi_{s^{N}}$ for each history $s^{N}$ is computed using the Euler equation for labor (115).

We also have a similar convergence result to that of Proposition 2.

Proposition 7 (Convergence of allocations) With similar notation to Proposition 2, we have the following convergence result for allocations:

$$
\left(c_{t, s^{N}}, a_{t, s^{N}}, l_{t, s^{N}}\right)_{s^{N}} \longrightarrow{ }_{N}\left(c_{t}\left(s^{\infty}\right), a_{t}\left(s^{\infty}\right), l_{t}\left(s^{\infty}\right)\right)_{s^{\infty} \in \mathcal{S}^{\infty}} \text {, almost surely. }
$$

Similarly, for preference shifters: $\xi_{s^{N}} \longrightarrow_{N} 1$ and $\xi_{s^{N}}^{l} \longrightarrow_{N} 1$, almost surely.

In other words, constructing a truncated model in the presence of a separable utility function is possible and its main properties do still hold. 
The dynamics. As in Section 4.1.3, we make the assumption that in the presence of aggregate risk, the $\xi \mathrm{s}$ and $\xi^{l} \mathrm{~s}$ remain constant and equal to their steady-state values. Similarly, the set of credit-constrained histories is assumed to be time-invariant. The resulting truncated model in the presence of aggregate shocks is then characterized by Euler equations (112) and (113) with $\xi_{s^{N}, t}=\xi_{s^{N}}$ and $\xi_{s^{N}, t}^{l}=\xi_{s^{N}}^{l}$ (the $\xi$ s remain equal to their steady-state values), as well as by the budget constraint (25), the market clearing conditions (29), the factor prices (3), and the UI budget constraint (14) (which remain unchanged compared to the GHH economy).

\section{E.3.3 The Ramsey program}

Formulation. We now formulate the Ramsey program in the presence of the separable utility function. The Ramsey program of equation (15) becomes:

$$
\max _{\left(\left(a_{t, s^{N}}, c_{t, s^{N}}, l_{t, s^{N}}\right)_{s \in \mathcal{S}^{N}}, \phi_{t}, \tau_{t}, r_{t}, w_{t}\right)_{t \geq 0}} \mathbb{E}_{0}\left[\sum_{t, s^{N}} \beta^{t} S_{t, s^{N}}\left(\xi_{s^{N}} u\left(c_{t, s^{N}}\right)-\xi_{s^{N}}^{l} v\left(\hat{l}_{t, s^{N}}\right)\right)\right]
$$

subject to Euler equations for (112) and (113), as well as the same set of equations as in the GHH case: (i) the budget constraints (25), (ii) the UI scheme budget balance (14), (iii) the market clearing constraints (29), and finally (iv) the factor prices (3).

Ramsey first-order conditions. To simplify the derivation of first-order conditions, we will also assume that the labor supply $\left(l_{t, s^{N}}\right)_{s^{N} \in \mathcal{S}^{N}}$ is a choice variable, in addition to savings choices $\left(a_{t, s^{N}}\right)_{s^{N} \in \mathcal{S}^{N}}$ and the replacement rate $\phi_{t}$ (already present in the GHH case).

The Lagrange multiplier for the consumption Euler equation is still $\beta^{t} S_{t, s^{N}} \lambda_{t, s^{N}}$, while the one for the labor Euler equation is $\beta^{t} S_{t, s^{N}} \mu_{t, s^{N}}$. We introduce $\tilde{\Psi}_{t, s^{N}}$, defined as:

$$
\tilde{\Psi}_{t, s^{N}}=\Psi_{t, s^{N}}+\left(1-\tau_{t}\right) F_{L, t} \mu_{t, s^{N}} \xi_{s^{N}} u^{\prime \prime}\left(c_{t, s^{N}}\right)
$$

which reflects the value of liquidity. In addition to the GHH case, it accounts for the fact 
that the labor supply diminishes if consumption increases. The FOC for saving choices is:

$$
\begin{aligned}
& \tilde{\Psi}_{t, s^{N}}=\beta \sum_{\tilde{s}^{N}} \mathbb{E}_{t}\left[\left(\left(1+r_{t+1}\right) \Pi_{t+1, s^{N}, \tilde{s}^{N}}+F_{K K, t+1} S_{t+1, \tilde{s}^{N}} \tilde{a}_{\tilde{s}^{N}, t}\right) \tilde{\Psi}_{t+1, \tilde{s}^{N}}\right] \\
& +\beta F_{K L, t+1} \sum_{\tilde{s}^{N}} \mathbb{E}_{t}\left[S_{t+1, \tilde{s}^{N}} \tilde{\Psi}_{t+1, \tilde{s}^{N}}\left(\left(1-\tau_{t+1}\right) 1_{\tilde{e}_{0}^{N}=e}+\frac{S_{t+1, e}}{S_{t+1, u}} \tau_{t+1} 1_{\tilde{e}_{0}^{N}=u}\right) \tilde{y}_{0}^{N} l_{t+1, \tilde{s}^{N}}\right] \\
& +\beta \sum_{\tilde{s}^{N}} \mathbb{E}_{t}\left[S_{t+1, \tilde{s}^{N}}\left(F_{K K, t+1} \tilde{\lambda}_{t+1, \tilde{s}^{N}}+\left(1-\tau_{t+1}\right) F_{K L, t+1} \mu_{t+1, \tilde{s}^{N}} \xi_{\tilde{s}^{N}}\right) \xi_{\tilde{s}^{N}} u_{t+1, \tilde{s}^{N}}^{\prime}\right] .
\end{aligned}
$$

The two first lines are very similar to the GHH case, while the last one includes a term that is specific to our separable utility function. The FOC with respect to labor supply is:

$$
\begin{aligned}
& \frac{\xi_{s^{N}}^{l}}{y_{0}}\left(v^{\prime}\left(l_{t, s^{N}}\right)+\mu_{t, s^{N}} v^{\prime \prime}\left(l_{t, s^{N}}\right)\right)-\left(\left(1-\tau_{t}\right) 1_{\tilde{e}_{0}=e}+\frac{S_{t, e}}{S_{t, u}} \tau_{t} 1_{\tilde{e}_{0}=u}\right) F_{L, t} \xi_{s^{N}} \tilde{\Psi}_{t, s^{N}} \\
& =\sum_{\tilde{s}^{N} \in \mathcal{S}^{N}} S_{t, \tilde{s}^{N}} \xi_{\tilde{s}^{N}} \tilde{\Psi}_{t, \tilde{s}^{N}}\left(F_{K L, t} \tilde{a}_{s^{N}, t-1}+\left(\left(1-\tau_{t}\right) 1_{\tilde{e}_{0}=e}+\frac{S_{t, e}}{S_{t, u}} \tau_{t} 1_{\tilde{e}_{0}=u} y_{0}\right) F_{L L, t} l_{t, \tilde{s}^{N}}\right) \\
& +\sum_{\tilde{s}^{N}} S_{t, \tilde{s}^{N}}\left(F_{K L, t} \tilde{\lambda}_{t, \tilde{s}^{N}}+\left(1-\tau_{t}\right) F_{L L, t} \mu_{t, \tilde{s}^{N}}\right) \xi_{\tilde{s}^{N}} u_{t, \tilde{s}^{N}}^{\prime}
\end{aligned}
$$

Finally, the first-order equation with respect to the replacement rate is:

$$
\sum_{s^{N} \in \mathcal{S}^{N}} S_{t, s^{N}} \mu_{t, s^{N}} \xi_{s^{N}} u^{\prime}\left(c_{t, s^{N}}\right)=\sum_{s^{N} \in \mathcal{S}^{N}} S_{t, s^{N}} \xi_{s^{N}} \tilde{\Psi}_{t, s^{N}}\left(-1_{e_{0}^{N}=e}+\frac{S_{t, e}}{S_{t, u}} 1_{e_{0}^{N}=u}\right) y_{0}^{N} l_{t, \tilde{s}^{N}},
$$

which balances the benefits of a higher replacement rate with its cost. The proofs for expressions (117)-(119) follows the exact sames lines as the one of Section 4.2 (with GHH utility function) and is skipped for the sake of conciseness.

The algorithm for simulation the Ramsey solution remains the same as the one of Section 4.2.2 in the GHH case, except that both the $\xi$ s and $\xi^{l}$ s have to be computed using the steady-state allocations (Step 4.a).

\section{E.3.4 Conclusion}

Section E.3 has shown that our truncation method can be readily extended to a more general utility function. The only difference is that due to the non-linearity of the Euler equation for labor supply, we need to include $\xi$ s that are specific to the labor Euler equation. The rest (including the Ramsey problem) is mostly unchanged. 